University of Tennessee Health Science Center UTHSC Digital Commons

\title{
The Role of the YXX $\Phi$ Motif in CD82 Trafficking and Function
}

Mekel Marie Richardson

University of Tennessee Health Science Center

Follow this and additional works at: https://dc.uthsc.edu/dissertations

Part of the Amino Acids, Peptides, and Proteins Commons, and the Neoplasms Commons

\section{Recommended Citation}

Richardson, Mekel Marie, "The Role of the YXXФ Motif in CD82 Trafficking and Function" (2012). Theses and Dissertations (ETD). Paper 219. http://dx.doi.org/10.21007/etd.cghs.2012.0259.

This Dissertation is brought to you for free and open access by the College of Graduate Health Sciences at UTHSC Digital Commons. It has been accepted for inclusion in Theses and Dissertations (ETD) by an authorized administrator of UTHSC Digital Commons. For more information, please contact jwelch30@uthsc.edu. 


\title{
The Role of the YXX $\Phi$ Motif in CD82 Trafficking and Function
}

\begin{abstract}
CD82, a tetraspanin, is a tumor metastasis suppressor. Tetraspanins are membrane spanning proteins that play critical roles in diverse biological and pathological processes, e.g., the regulation of cancer metastasis. CD82 is ubiquitously expressed in various types of tissues, but its expression becomes downregulated or lost in a majority of metastatic tumors. It inhibits tumor metastasis without affecting primary tumor growth. Cancer patients whose tumors contain CD82 exhibit minimal metastasis. We know that CD82 functions as a tumor metastasis suppressor but the mechanism by which this occurs is largely unknown. CD82 can be found on the plasma membrane as well as in intracellular compartments, and CD82 undergoes endocytosis. The intracellular trafficking of proteins plays a key role in their functions. The mechanism by which CD82 is able to traffic remains not well known. Notably, CD82 contains an YXXФ internalization and intracellular trafficking motif in its C-terminal cytoplasmic domain.
\end{abstract}

Previous reports suggest that the mutation of the $Y X X \Phi$ motif of a tetraspanin will disrupt internalization and subsequent trafficking. We have established the stable transfectant of CD82 YVAA mutant, which contains a mutation in the YXXФ motif of CD82.

This research focuses on the roles of CD82's YXXФ motif 1) in intracellular trafficking of CD82 and its associated proteins as well as 2) in the CD82-mediated inhibition of the migration of metastatic tumor cells. Results from the internalization assays reveal that the YXXФ motif is not required for the internalization of CD82, nor does the mutation of this motif have drastic effect on the steady state distribution of CD82. Results from the intracellular trafficking and recycling assays reveal that there is a defect in the intracellular trafficking of CD82 upon the mutation of its YXXФ motif. We found that both CD82 WT and CD82 YVAA mutant trafficked to the late endosomes/lysosomal compartments. We also observed that the recycling of CD82 proteins is Rab 11-mediated process and that the mutation of the YXXФ motif significantly decreases the CD82 YVAA release via exosomes. Our study also revealed that the CD82 YVAA mutant traffics to and accumulates within the lysosomal compartments within the cells. Regarding the role of CD82 trafficking in cell movement, we found that that the mutation of CD82 YXXФ motif results in a loss of function in CD82-mediated inhibition of collective cell migration and cell invasion but appears not to affect CD82-mediated inhibition of solitary cell migration. In addition, CD82 YVAA mutation increases cell-cell adhesion in a calcium dependent manner, which likely results from altered trafficking of cell-cell adhesion proteins.

From the results obtained in this study, we conclude that the YXXФ motif of CD82 is not essential for CD82 internalization but rather regulates CD82 trafficking to the late endosome where CD82 is recycled back to the plasma membrane or secreted into the extracellular environment via exosomes. CD82 trafficking also regulates the trafficking and function of cell adhesion proteins. Furthermore, the proper trafficking and recycling of CD82 plays a key role in maintaining its motility suppressive function. Further studies will determine 1) the molecular mechanism by which CD82 trafficking regulates the behavior of cell adhesion proteins and 2) the effect of aberrant CD82 trafficking on tumor metastasis in animal models.

\section{Document Type}

Dissertation

\section{Degree Name}

Doctor of Philosophy (PhD) 


\section{Program}

Biomedical Sciences

Research Advisor

Xin Zhang, M.D.

\section{Keywords}

CD82, internalization, intracellular trafficking, tetraspanin, YXXФ motif

\section{Subject Categories}

Amino Acids, Peptides, and Proteins | Chemicals and Drugs | Diseases | Medicine and Health Sciences | Neoplasms 


\title{
THE ROLE OF THE YXXФ MOTIF IN CD82 TRAFFICKING AND FUNCTION
}

\author{
A Dissertation \\ Presented for \\ The Graduate Studies Council \\ The University of Tennessee \\ Health Science Center
}

\author{
In Partial Fulfillment \\ Of the Requirements for the Degree \\ Doctor of Philosophy \\ From The University of Tennessee
}

By

Mekel Marie Richardson

December 2012 
Copyright (C) 2012 by Mekel Marie Richardson. All rights reserved. 


\section{DEDICATION}

This is dedicated to my loving son Jayden Travis, my nephew Brandon Coleman and In Loving Memory of my mother Mrs. Gloria Marie Richardson and brother Mr. William Glen Richardson. 


\section{ACKNOWLEDGEMENTS}

I would like to thank my mentor, Xin A Zhang, and committee members, Lisa K Jennings, Andrzej Slominski, John Cox and Eldon Geisert, for all of their guidance, advice, patience, and encouragement during my PHD studies.

I am grateful to my colleagues, Yanhui Zhang, Feng Zhang, Li Liu, Oulimata Kane-Grossman, and Chenying Fu for their valuable collaborations, discussions, and friendship.

During the course of my PhD studies, I was supported in part by the NIH Diversity Supplement Grant number CA096991. 


\begin{abstract}
CD82, a tetraspanin, is a tumor metastasis suppressor. Tetraspanins are membrane spanning proteins that play critical roles in diverse biological and pathological processes, e.g., the regulation of cancer metastasis. CD82 is ubiquitously expressed in various types of tissues, but its expression becomes down-regulated or lost in a majority of metastatic tumors. It inhibits tumor metastasis without affecting primary tumor growth. Cancer patients whose tumors contain CD82 exhibit minimal metastasis. We know that CD82 functions as a tumor metastasis suppressor but the mechanism by which this occurs is largely unknown. CD82 can be found on the plasma membrane as well as in intracellular compartments, and CD82 undergoes endocytosis. The intracellular trafficking of proteins plays a key role in their functions. The mechanism by which CD82 is able to traffic remains not well know. Notably, CD82 contains an YXXФ internalization and intracellular trafficking motif in its C-terminal cytoplasmic domain. Previous reports suggest that the mutation of the YХХФ motif of a tetraspanin will disrupt internalization and subsequent trafficking. We have established the stable transfectant of CD82 YVAA mutant, which contains a mutation in the YXXФ motif of CD82.
\end{abstract}

This research focuses on the roles of CD82's YXXФ motif 1) in intracellular trafficking of CD82 and its associated proteins as well as 2) in the CD82-mediated inhibition of the migration of metastatic tumor cells. Results from the internalization assays reveal that the YXXФ motif is not required for the internalization of CD82, nor does the mutation of this motif have drastic effect on the steady state distribution of CD82. Results from the intracellular trafficking and recycling assays reveal that there is a defect in the intracellular trafficking of CD82 upon the mutation of its YXXФ motif. We found that both CD82 WT and CD82 YVAA mutant trafficked to the late endosomes/lysosomal compartments. We also observed that the recycling of CD82 proteins is Rab 11-mediated process and that the mutation of the YXXФ motif significantly decreases the CD82 YVAA release via exosomes. Our study also revealed that the CD82 YVAA mutant traffics to and accumulates within the lysosomal compartments within the cells. Regarding the role of CD82 trafficking in cell movement, we found that that the mutation of CD82 YXXФ motif results in a loss of function in CD82-mediated inhibition of collective cell migration and cell invasion but appears not to affect CD82-mediated inhibition of solitary cell migration. In addition, CD82 YVAA mutation increases cell-cell adhesion in a calcium dependent manner, which likely results from altered trafficking of cell-cell adhesion proteins.

From the results obtained in this study, we conclude that the YXXФ motif of CD82 is not essential for CD82 internalization but rather regulates CD82 trafficking to the late endosome where CD82 is recycled back to the plasma membrane or secreted into the extracellular environment via exosomes. CD82 trafficking also regulates the trafficking and function of cell adhesion proteins. Furthermore, the proper trafficking and recycling of CD82 plays a key role in maintaining its motility suppressive function. Further studies will determine 1) the molecular mechanism by which CD82 trafficking 
regulates the behavior of cell adhesion proteins and 2) the effect of aberrant CD82 trafficking on tumor metastasis in animal models. 


\section{TABLE OF CONTENTS}

CHAPTER 1. INTRODUCTION .1

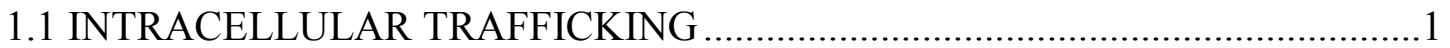

1.2 REGULATION OF INTRACELLULAR TRAFFICKING …..................................

1.2.1 The endosomal and lysosomal trafficking ...................................................

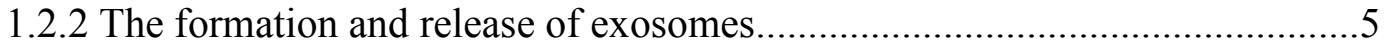

1.3 CELL-CELL ADHESION ........................................................................

1.4 TETRASPANINS AND TETRASPANIN-ENRICHED MICRODOMAINS

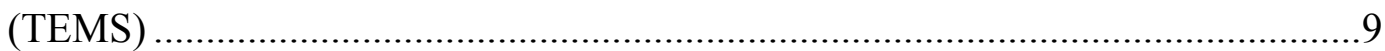

1.4.1 Tetraspanins

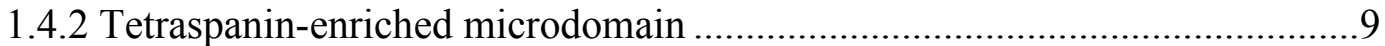

1.5 THE INTRODUCTION OF TETRASPANIN CD82 ……...............................10

1.5.1 The chromosomal locus of the $C D 82$ gene...............................................10

1.5.2 The expression and regulation of the $C D 82$ gene.......................................10

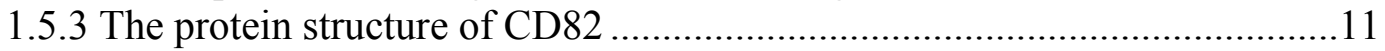

1.5.4 Tissue distribution of CD82 …….........................................................13

1.6 THE PATHOLOGICAL FUNCTIONS OF CD82: CD82 AND TUMOR

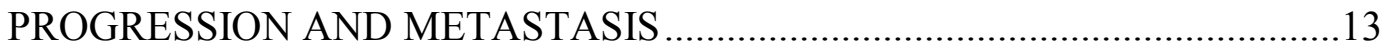

1.7 CD82-CONTAINING TETRASPANIN-ENRICHED MICRODOMAIN ............14

1.8 CD82 AND CELL-CELL ADHESION .........................................................15

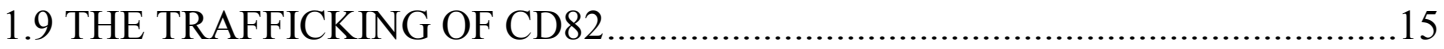

\section{CHAPTER 2. REGULATION OF TRAFFICKING BY THE YXXФ MOTIF OF}

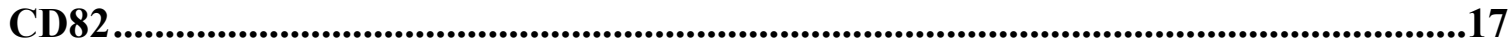

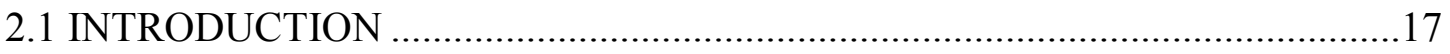

2.2 MATERIALS AND METHODS .............................................................18

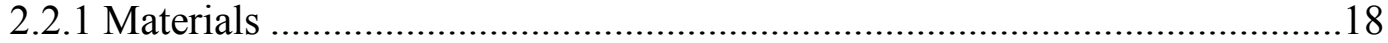

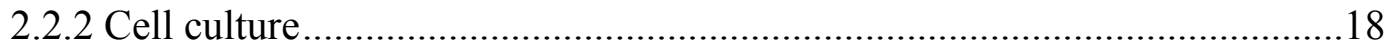

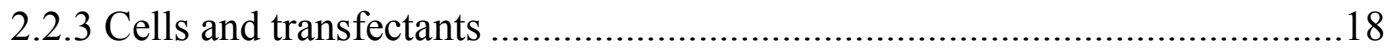

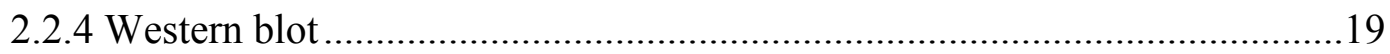

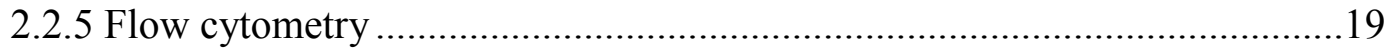

2.2.6 Internalization and recycling assays ....................................................19

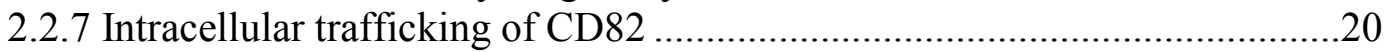

2.2.8 Fluorescent and confocal microscopy......................................................21

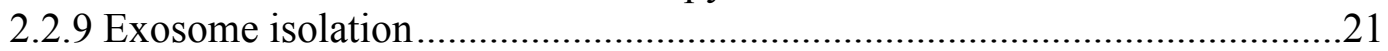

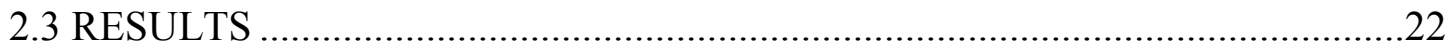

2.3.1 Generation of stable CD82 WT and CD82 YVAA transfectants in PC3

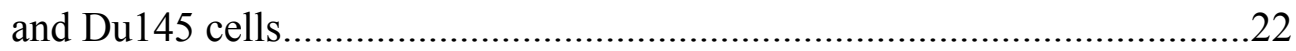

2.3.2 Mutation of the YXXФ motif of CD82 did not alter the steady state

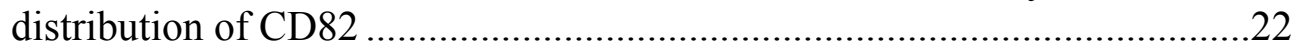

2.3.3 Mutation of the YXXФ motif of CD82 does not abrogate CD82

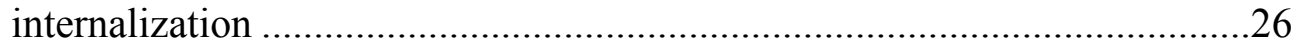

2.3.4 The mutation of the YХXФ motif of CD82 alters CD82 recycling..............26 
2.3.5 The mutation of the YXXФ motif does not disrupt the trafficking of CD82 to the late endosomal and lysosomal compartments

2.3.6 CD82 proteins are likely recycled by Rab 11 recycling endosomes and the YVAA mutation reduced CD82 trafficking to Rab 11 recycling endosomes.....

2.3.7 CD82 proteins are released from PC3 cells via exosomes

2.3.8 The mutation of the YXXФ motif of CD82 does not affect the cell surface expression levels of CD82-associated tetraspanins but upregulates the cell surface expression levels of integrins $\alpha 2 \beta 1, \alpha 3 \beta 1$, and $\alpha 5 \beta 1$

2.3.9 The mutation of CD82 YXXФ motif alters the trafficking of integrins $\alpha 3 \beta 1, \alpha 6 \beta 1$, and $\alpha 5 \beta 1$ into CD82-positive compartments

\section{CHAPTER 3. THE YXXФ MOTIF-MEDIATED REGULATION OF THE} INTRACELLULAR TRAFFICKING OF CD82 ....................................................48

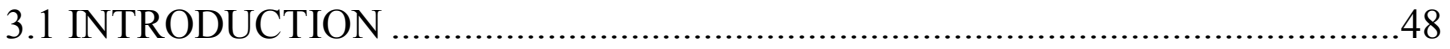

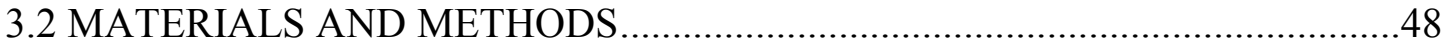

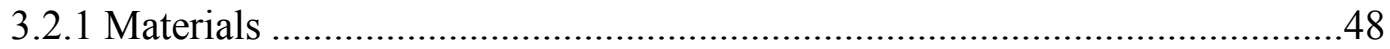

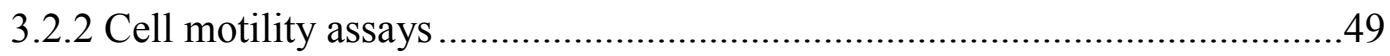

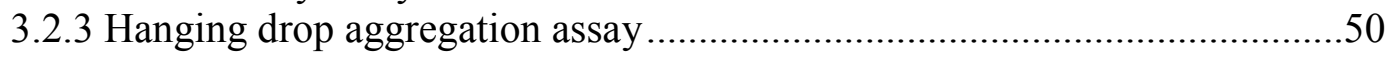

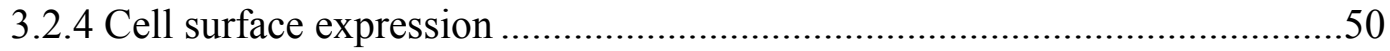

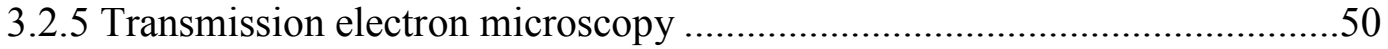

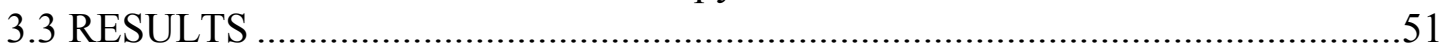

3.3.1 The mutation of the YXXФ motif does not abolish CD82-mediated inhibition of solitary cell migration .....................................................51

3.3.2 The mutation of the YXXФ motif abolished the motility-suppressive function of CD82 in collective cell migration .........................................51

3.3.3 The mutation of the YXXФ motif of CD82 leads to an increase in CD82 mediated cell-cell adhesion...................................................................56

3.3.4 CD82 YVAA transfectants probably increase desmosome-mediated cellcell adhesion....

CHAPTER 4. DISCUSSION .........................................................................................66

4.1 INTRODUCTION

4.2 THE YXXФ MOTIF IS NOT ESSENTIAL FOR CD82 INTERNALIZATION .67

4.3 THE MUTATION OF THE CD82 YXXФ MOTIF LEADS TO THE

ATTENUATION AND ALTERATION IN CD82 RECYCLING

4.4 THE MUTATION OF THE CD82 YXXФ MOTIF DISRUPTS

INTRACELLULAR TRAFFICKING OF CD82 AND CAUSES THE

MUTATED CD82 PROTEINS TO ACCUMULATE IN LYSOSOMES .

4.5 THE MUTATION OF THE CD82 YXXФ REDUCED THE EXOSOMAL

SECRETION OF CD82 PROTEINS.

4.6 THE PROPER INTRACELLULAR TRAFFICKING OF CD82 IS NEEDED

FOR CD82-MEDIATED INHIBITION OF CELL MOTILITY. 
4.7 THE MUTATION OF THE YXXФ MOTIF IN CD82 ALTERS CELL-CELL

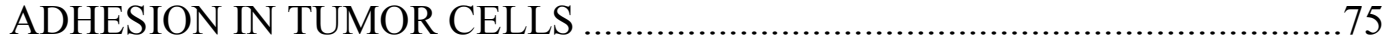

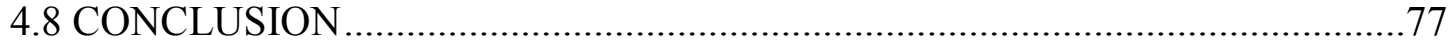

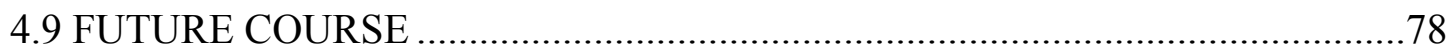

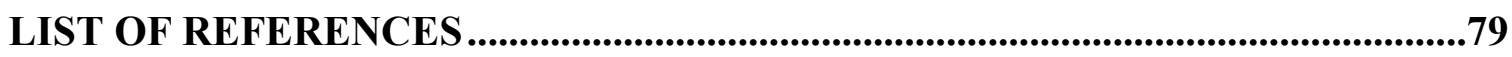

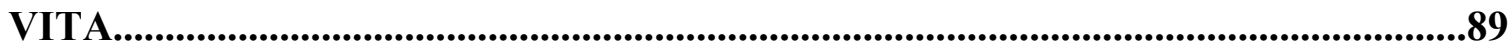




\section{LIST OF FIGURES}

Figure 1-1. The genomic localization of the CD82 gene..............................................11

Figure 1-2. The protein structure of the tetraspanin CD82 _.......................................12

Figure 2-1. The expression of CD82 proteins in PC3 transfectant cells were analyzed by Western blot

Figure 2-2. The expression of CD82 proteins at the surface of PC3 transfectant cells were analyzed by flow cytometry

Figure 2-3. The mutation of the YXXФ motif of CD82 does not result in an alteration of steady state distribution of CD82 proteins in PC3 cells

Figure 2-4. The mutation of the YXXФ motif of CD82 does not significantly reduce CD82 internalization

Figure 2-5. The mutation of the YXXФ motif of CD82 does not reduce but may enhance CD82 internalization .28

Figure 2-6. The YXXФ motif regulates CD82 recycling .................................................29

Figure 2-7. Mutation of the YXXФ motif regulates CD82 recycling .............................30

Figure 2-8. The mutation of the YXXФ motif does not disrupt CD82 trafficking to CD63-positive late endosomal and lysosomal compartments

Figure 2-9. The mutation of the YXXФ motif does not disrupt CD82 trafficking to the multivesicular bodies (MVBs) of the cells

Figure 2-10. Mutation of the YXXФ motif directs CD82 trafficking to the lysosomal compartments of the cells

Figure 2-11. Mutation of the YXXФ motif disrupts CD82 trafficking to the Rab 11 positive compartments

Figure 2-12. The effects of CD82 overexpression and CD82 YVAA mutation on exosomal content.

Figure 2-13. Mutation of the YXXФ motif of CD82 does not affect the cell surface expression of CD82-associated tetraspanins but up-regulates the cell surface levels of integrins $\alpha 2, \alpha 3$, and $\alpha 5$ in PC 3 cells

Figure 2-14. Mutation of the YXXФ motif of CD82 does not affect the number of vesicles per cell or the colocalization of $\alpha 2$ with CD82 after internalization. 
Figure 2-15. Mutation of the $\mathrm{YXX \Phi} \mathrm{motif} \mathrm{of} \mathrm{CD82} \mathrm{leads} \mathrm{to} \mathrm{an} \mathrm{accumulation} \mathrm{of} \alpha 3$ vesicles after internalization

Figure 2-16. Mutation of the YXXФ motif of CD82 leads to an accumulation of $\alpha 5$ vesicles after internalization

Figure 2-17. Mutation of the YXXФ motif of CD82 leads to an increase in CD82/ $\alpha 6$

colocalization after internalization

Figure 3-1. Mutation of the YXXФ motif does not disrupt CD82-mediated inhibition of solitary cell movement

Figure 3-2. The mutation of the YXXФ motif causes a loss of CD82- mediated inhibition in collective cell migration in PC3 cells

Figure 3-3. The mutation of the YXXФ motif causes a loss of CD82-mediated inhibition in collective cell migration in Du145 cells

Figure 3-4. The mutation of the YXXФ motif of CD82 attenuates CD82-mediated inhibition of cell invasion

Figure 3-5. The YХXФ mutation attenuates CD82-mediated inhibition of total cell-cell aggregation in $\mathrm{PC} 3$ cells

Figure 3-6. Mutation of the YXXФ motif of CD82 attenuates CD82-mediated inhibition of total cell-cell aggregation in PC3 cells .58

Figure 3-7. The YXXФ mutation does not affect calcium-independent cell-cell aggregation in $\mathrm{PC} 3$ cells

Figure 3-8. The YXXФ mutation decreases calcium-independent cell-cell aggregation in PC3 cells

Figure 3-9. The effects of CD82 overexpression and CD82 YVAA mutation on cell-cell aggregation in Du145 cells

Figure 3-10. The mutation of the YXXФ motif of CD82 leads to an increase in the cell surface expression of cell adhesion molecule EpCAM

Figure 3-11. The mutation of the YXXФ motif in CD82 attenuated CD82-dependent reduction in the formation of cell-cell adhesions 


\section{CHAPTER 1. INTRODUCTION}

\subsection{INTRACELLULAR TRAFFICKING}

Endocytosis is the mechanism by which cells uptake their nutrients and solutes from the surrounding environment. The process of endocytosis is used by all cells of the body and regulates numerous biological processes such as regulation of cell surface receptors, maintaining the homeostasis of cellular cholesterol, antigen presentation, and maintenance of cellular processes (Xu et al 2009). Endocytosis can be classified into two major categories: phagocytosis and pinocytosis.

Phagocytosis is the process by which cells bind and internalize particulate matter larger than around $0.75 \mu \mathrm{m}$ in diameter, such as small-sized dust particles, cell debris, micro-organisms and even apoptotic cells, which only occurs in specialized cells (Conner and Schmid 2003; Soldati and Schliwa 2006; Xu et al 2009).

Pinocytosis is a "cell drinking' process that uptakes small particles and solutes (Conner and Schmid 2003; Soldati and Schliwa 2006). Pinocytosis can further be subdivided into 4 subclasses, clathrin-mediated endocytosis, caveolae-mediated endocytosis, macropinocytosis, and clathrin and caveolae independent endocytosis (Alberts et al 2002; Conner and Schmid 2003).

Clathrin-mediated endocytosis is mediated by the small vesicles that are approximately $100 \mathrm{~nm}$ in diameter and a morphologically characteristic crystalline coat made up of a complex of cytosolic proteins, clathrin and its associated proteins. These small vesicles are called clathrin-coated vesicles (CCVs) and are found in virtually all cells. Before CCVs are formed, clathrins and their associated proteins cluster at the plasma membrane to form membrane microdomains and cause the local invagination of the plasma membrane termed clathrin-coated pits. The clathrin-coated pits can concentrate the large extracellular molecule ligands, which bind their transmembrane receptors to trigger the clathrin-mediated endocytosis of these ligands. These extracellular ligands include low-density lipoprotein, transferrin, growth factors, and antibodies.

Caveolae are the most commonly reported non-clathrin-coated plasma membrane buds, which exist on the cell surface of many, but not all, cell types. They consist of the cholesterol-binding protein caveolin (Vip21). Caveolin is localized in the lipid bilayer and is enriched in cholesterol and glycolipids. Caveolae are small, approximately $50 \mathrm{~nm}$ in diameter, flask-shape pits in the plasma membrane that resemble the shape of caves, hence termed as caveolae (Conner and Schmid 2003). They can constitute up to one third 
of the plasma membrane of the cells in some tissues, being especially abundant in smooth muscle, type I pneumocytes, fibroblasts, adipocytes, and endothelial cells. The uptake of extracellular molecules such as albumin is believed to be mediated via caveolae.

Macropinocytosis usually occurs at highly ruffled regions of the plasma membrane (Conner and Schmid 2003). Macropinocytosis starts from the invagination of the local plasma membrane to form a pocket, which then pinches off into the cell to form a relatively large vesicle, $0.5-5 \mu \mathrm{m}$ in diameter, filled with a large volume of extracellular fluid and soluble molecules, equivalent to $100 \mathrm{CCVs}$ in volume. The filling of the pocket occurs in a non-specific manner. The macropinocytic vesicle then travels into the cytosol and fuses with other intracellular vesicles such as endosomes and lysosomes.

The clathrin- and caveola-independent endocytosis involves a group of poorly defined mechanisms. Some clathrin- and caveola-independent endocytosis appears to use lipid rafts or lipid raft-like domains as the membrane platform to initiate endocytosis. Lipid rafts are small cholesterol rich microdomains that freely diffuse on the cell surface (Conner and Schmid 2003). Their unique lipid composition provides a physical basis for specific sorting of membrane proteins and/or glycolipids based on their transmembrane regions (Conner and Schmid 2003). An earlier study from our lab revealed that tetraspanin CD82 undergoes clathrin- and caveola-independent endocytosis, lipid raft mediated endocytosis (Xu et al 2009).

\subsection{REGULATION OF INTRACELLULAR TRAFFICKING}

Proteins are endocytosed by several different mechanisms. Once across the plasma membrane, the proteins are sorted/targeted to different organelles within the cell (Bonifacino and Traub 2003; Conner and Schmid 2003; Escola et al 1998; Gruenberg 2001; Kang and Creswell 2002; Kerkhof et al 2001; Lakkaraju and Boulan-Rodriguez 2008; Lodish et al 2004; Mukherjee et al 1997; Savina et al 2003; Traub 2003; Wessling-Resnick and Braell, 1990). One of the deciding factors in the protein's destiny is the type of sorting motif that they contain (Lakkaraju and Boulan-Rodriguez 2008).

The most studied sorting motifs are the delucine based and tyrosine based sorting motifs (Brown and Brenton 2000). Both of these sorting motifs are found within the cytoplasmic tails of numerous proteins and are important regulators of intracellular trafficking and sorting (Brown and Brenton 2000). The delucine based sorting motif has been show to regulate protein trafficking to the trans-golgi network, to the lysosomes and is important for the internalization of numerous proteins (Bonfacino and Traub 2003). 
Tyrosine-based sorting motif, YXXФ, mediates the targeting of many integral membrane proteins of the secretory pathway. Within the YXXФ amino acid sequence, the $Y$ represents the tyrosine residue and studies have shown that the tyrosine residue is responsibe for the function of the motif (Bonfacino and Traub 2003; Marks et al 1997). These studies revealed that mutation or replacement of the tyrosine residue with any other residue led to a defect in the recognition of the motif (Bonfacino and Traub 2003). The $\Phi$ residue represents a bulky hydrophobic residue and can be substituted with any of the hydrophobic amino acids without a functional consequence (Bonfacino and Traub 2003; Marks et al 1997; Renard et al 2010). The X residue can be substituted with any of the amino acids.

Transmembrane proteins, such as tetraspanins contain the tyrosine based sorting motif, YXXФ (Bonifacino and Traub 2003; Kang and Cresswell 2002; Tonoli and Barrett 2005). In fact, 13 out of the 33 tetraspanin superfamily members contain the YXXФ motif (Berditchevski et al 2007). This motif plays a role in the 1) rapid internalization of the protein from the plasma membrane, and/or 2) targeting them to the endosomes and lysosomes (Berditchevsky and Odintsina 2007; Bonifacino and Traub 2003).

The transmembrane proteins that contain the YXXФ motif use the clathrin-coated pit as their mode of endocytosis (Conner and Schmid 2003; Traub 2003). The proteins using clathrin-coated pits interact with AP2 complexes, mainly $\mu 2$ subunit, through their tyrosine-based sorting motifs (Conner and Schmid 2003; Traub 2003).

Earlier studies demonstrated that the mutations of the $\mathrm{Y}$ or the $\Phi$ residues of this motif abolishes the endocytosis and subsequent the trafficking of the transmembrane proteins that carry this motif (Bonifacino and Traub 2003; Guarnier 1993; Liu et al 2007; Murkherjee et al 1997). Tetraspanins are a superfamily of type III transmembrane proteins that span the membrane four times. Some tetraspanins such as CD9, CD63, CD82, and CD151 contain the YXXФ trafficking motif in the C-terminal cytoplasmic domains. In order to shed light on the trafficking mechanism of tetraspanins, the YXXФ motifs of CD63 and CD151 have been mutated (Liu et al 2007; Rous et al 2002). In NIH $3 \mathrm{~T} 3$ cells, mutation of the YXXФ motif of CD151 led to the abrogation of CD151 internalization and subsequent trafficking (Liu et al 2007). Similar results were observed in normal rat kidney cells upon mutation of CD63 (Rous et al 2002). Also, another body of evidence comes from reports that patients with familial hypercholesterolemia, containing a mutation in the YXXФ motif of the low density lipid protein (LDL) receptor, exhibit a defect in the internalization of LDL (Mukherjee et al 1997). Tetraspanin CD82, which contains the YXXФ motif in the C-terminal domain, is localized at the plasma membrane as well as in intracellular vesicles (Liu and Zhang 2006; Miranti 2008). Although an earlier study from our lab demonstrated that the 
endocytosis of CD82 is independent of clathrin-mediated endocytosis, there have not been any reports which have addressed the function of the YXXФ motif of CD82.

\subsubsection{The endosomal and lysosomal trafficking}

Another deciding factor for the intracellular trafficking of proteins is which Rab GTPase proteins the proteins will encounter. Rab proteins are a family of ubiquitously expressed proteins that are essential for directing the various steps of intracellular trafficking. Once the protein is internalized, it is delivered to the endosomes, with the aid of Rab 5 (Kerkhof et al 2001; Lakkaraju and Boulan-Rodriguez 2008; Pols and Klumperman 2008). The endosomes are divided into the following categories: early, late, or recycling endosomes. The early endosomes are responsible for directing internalized proteins to their final fates, either being recycled or degraded. Proteins that leave the early endosomes and go to the late endosomes are usually sorted to the lysosome for degradation (Kerkhof et al 2001; Wessling-Resnick and Braell 1990). Those that leave the early endosomes and report to the recycling endosomes are recycled back to the plasma membrane (Bonifacino and Traub 2003; Lakkaraju and Boulan-Rodriguez 1990; Mukherjee et al 1997).

Some late endosomes accumulate internalized vesicles within their lumen and ultimately mature into the multivesicular bodies (MVBs), a form of late endosomes (Delcayre et al 2005; Duijvesz et al 2011; Escola et al 1998; Palanisamy et al 2010; Pols and Klumperman 2008; Savina et al 2003; Simpson et al 2008; Stoorvogel 2002). Once proteins reach the late endosome/multivesicular bodies, they have two fates: either being sorted to the lysosome for degradation or being secreted into the extracellular space after the MVBs fuses with the plasma membrane (Stoorvogel et al 2002). An example of proteins targeted for lysosomal degradation is growth factor receptors such as epidermal growth factor receptors, EGFR, and the lysosomal targeting thereby shuts down its signaling processes (Felder et al 1990).

The sorting of proteins into MVBs is a highly regulated process and has been shown to involve ubiquitination (Record et al 2011). Generally the proteins targeted for degradation are tagged with ubiquitin, termed ubiquitination, and trafficked to the multivesicular bodies for further lysosomal targeting and degradation (Alberts et al 2002; Hislop and von Zastrow 2011; Marsh and van Meer 2008). The trafficking of ubiquitin-tagged proteins to MVBs uses either an endosomal sorting complex required for transport (ESCRT), dependent or independent mechanism.

An important regulator for protein sorting and multivesicular body formation is ESCRT (Record et al 2011). During intracellular trafficking, the ESCRT machinery is 
recruited to the cytosolic side of the endosomal membrane thus allowing the proper sorting of selected proteins to the interluminal vesicles, which ultimately leads to the formation of the MVBs (Katzmann et al 2002; Record et al 2011). The ESCRT machinery is composed of three protein complexes, ESCRT-I, ESCORT-II, and ESCRT-III (Record et al 2011). The Vps27/Hrs complex recruits Tsg101, an ESCRT-1 complex member. Tsg101 binds the ubiquitinated proteins and activates the ESCRT-II complex. Tsg101 further recruits the ESCRT-III complex via ESCRT II and Alix (Record et al 2011). The ESCRT-III complex concentrates and plays a role in the formation of the multivesicular bodies by recruiting an enzyme that removes the ubiquitin tag from the protein prior to the protein being sorted into the multivesicular bodies (Février and Raposo 2004; Record et al 2011). It has also been shown that knock-down of Hrs and Tsg101 resulted in reduced trafficking of EGF to the interluminal vesicles, ILVs, of the MVBs (Trajkovic et al 2008).

For the ESCRT-independent sorting mechanism, the ubiquitinated protein is incorporated into detergent-resistant complexes, as in the case for MHC class II proteins. In immature dendritic cells, the sorting of MHC class II proteins to the MVBs is largely dependent upon the interaction of dendritic cells with cognate $\mathrm{T}$ cells. The resulting interaction is that ubiquitination is blocked and the MHC class II proteins are sorted together with the tetraspanin CD9 into MVBs (Buschow et al 2009). The tetraspanin superfamily of proteins, highly enriched in exosomes, has a high affinity for cholesterol and sphingolipids such as ceramide, a precursor for exosome release which potentially may create domains destined for the MVBs (de Gassat et al 2003). For example, in Oli-neu cells the proteolipid protein PLP is segregated into distinct subdomains and are transferred to exosomes in a raft-based, ceramide-dependent, and ESCRT-independent pathway (Record et al 2011; Trajkovic et al 2008). Tetraspanins also associates with and sequesters other cell surface proteins into membrane complexes at the plasma membrane such as other tetraspanins, integrin, and growth factor receptors. The ESCRT-independent sorting of MHC class II proteins provides a putative avenue for the MVB trafficking and exosomal secretion of other transmembrane proteins. Other proteinprotein interactions with the Alix and heat-shock proteins HSP 70 and HSC 70 allow proteins to traffic to the exosomes (Record et al 2011).

\subsubsection{The formation and release of exosomes}

It is noteworthy to mention that not all proteins destined for late endosomes are to be degraded, some of the late endosomes, especially MVBs, fuse with the plasma membrane and release their contents in the form of small vesicles, deemed exosomes, to the extracellular environment (Escola et al 1998; Pols and Klumperman 2008; Savina et al 2003). Exosomes are the nano-sized vesicles that were identified during the study of 
reticulocyte maturation (Harding et al 1983; Pan and Johnstone 1983). Since then, exosomes have been detected to be secreted from nearly all cells types including dendritic cells, macrophages, lymphocytes, salivary gland epithelial cells, and cancer cells (Delcayre et al 2005; Duijvesz et al 2011; Escola et al 1998; Heijene et al 1999; Palanisamy et al 2010; Pols and Klumperman 2008; Savina et al 2003; Simpson et al 2008; Stoorvogel 2002). Exosomes are also detected in bodily fluid such as saliva, plasma, urine, breast milk, amniotic fluid, synovial fluid, and bronchoaveolar lavage fluid (Record et al 2011).

Exosome biogenesis initially stems from endocytosis and is largely regulated by the formation of MVBs (Delcayre et al 2005; Duijvesz et al 2011; Escola et a 11998 ; Heijene et al 1999; Palanisamy et al 2010; Pols and Klumperman 2008; Savina et al 2003; Simpson et al 2008; Stoorvogel 2002), as discussed above.

The secretion of exosomes is regulated by a different set of molecules. To date the major regulator of exosome secretion is the Rab GTPase family of proteins. Exosome secretion has been shown to be regulated by Rab27a and Rab27b (Ostrowski et al 2010; Pfeffer 2010). It was determined that the knock-down of Rab27a and Rab27b decreased exosome secretion without effecting exosome composition (Ostrowski et al 2010). In addition, Savina et al reported that Rab 11 also plays a role in the exosome pathway (Savina et al 2003). Moreover, exosome secretion is also regulated by the lipid ceramide (Trajkovic et al 2008). Ceramide is generated by the hydrolysis of the phosphocholine moiety of sphingomyelin (SM) by sphingomyelinases (Smases) (Trajkovic et al 2008). Inhibition of ceramide by the treatment with a sphingomyelinase (nSMase) inhibitor GW4869 in Oli-neu cells led to a decrease in exosome secretion (Trajkovic et al 2008). It was also observed that treatment with an exogenous sphingomyelinase or ceramide led to an increase in the production and release of exosomes in the Oli-neu cells (Trajkovic et al 2008).

Not surprising, exosomes and MVBs have similar molecular compositions (Duijvesz et al 2011; Record et al 2011; Stoorvogel et al 2002; Trajkovic et al 2008). There are many common proteins found in exosomes and MVBs, however the overall composition of exosomes depends largely on the cells from which they originate (Record et al 2011; Stoorvogel et al 2008). Common proteins found within exosomes are Alix, flotillin-1, heat shock proteins such as Hsp70, Hsp90, and Hsc 70, LBPA, lipids such as ceramide, tetraspanins such as CD9, CD63, CD81, CD82, GPI-anchored proteins such as TNFR-1, antigen presenting molecules MHC-I and MHC-II, cell adhesion molecules such as integrins, cadherins, and I-CAM, luminal proteins such as annexin 2 and cytokines, ESCRT machinery proteins such as Tsg101, Alix, and Hrs, and the proteins involved in transcription, protein synthesis, trafficking, and signaling such as histones, 
ribosomal proteins, ubiquitin, ARF and Rab GTPase family members, G proteins, RhoA, and kinases (Duijvesz et al 2011; Record et al 2011; Théry et al 1999; Théry et al 2001). Exosomes also contain non-protein materials such as mRNA and microRNAs (miRNA) (Duijvesz et al 2011; Mathivanan and Simpson 2009; Record et al 2011; Théry et al 2001; Valadi et al 2007). In fact, there are at least 2400 proteins, 900 mRNA, and 275 miRNA found in exosomes to date (Mathivanan and Simpson 2009).

\subsection{CELL-CELL ADHESION}

Cell-cell adhesions are the characteristic of multi-cellular organisms (Alberts et al 2002). Cell-cell adhesion plays crucial roles in a wide range of physiological and pathological functions ranging from sperm-egg fusion, organogenesis, tissue stability, inflammation, leukocyte rolling, collective cell movement, and tumor metastasis. In collective cell migration, a cohort of cells form cell-cell contact with each other and move together. In this process, cell-cell adhesion coordinates the behavior of individual cells and connects them into a migratory unit.

Cell-cell adhesion can be classified into calcium-dependent and calcium-independent cell-cell adhesion events based on the requirement of bivalent cation calcium in the formation of cell-cell contacts (Lodish et al 2004). The membrane proteins responsible for calcium-dependent cell-cell adhesion include cadherins and selectins, while the proteins such as immunoglobulin superfamily (IgSF) proteins are responsible for calcium-independent cell-cell adhesion (Lodish et al 2004).

Cadherins are the major cell-cell adhesion proteins (CAMs) responsible for $\mathrm{Ca} 2+$ dependent cell-cell adhesion in vertebrate tissues and are expressed on virtually all cell types. Cadherins are single-pass transmembrane glycoproteins, and each cadherin contains approximately 100-750 amino acids. Cadherins associate with each other in the plasma membrane to form dimers or oligomers. The large extracellular part of the polypeptide chain is usually folded into five or six cadherin repeats and have calcium ions positioned between each pair of repeats. This cadherin-calcium orientation creates a stiff and rigid structure resulting in stronger cell-cell adhesions. In cell culture, the removal of extracellular $\mathrm{Ca} 2+$ or treatment with anti-cadherin antibody disrupts cell-cell adhesion, and the protein is rapidly degraded by proteolytic enzymes (Lodish et al 2004).

Most cadherins are normally concentrated in adherens junctions, which are connected to cortical actin cytoskeletons of the cells and hold cells together in a homotypic cell-cell adhesion manner. Some cadherins such as desmoglein and desmocollin form desmosomes, which are connected to the intermediate filament cytoskeleton and often found in epithelial tissues (Lodish et al 2004). Cadherins can be 
grouped into classical and non-classical cadherins. There are four major classical cadherins: E-cadherins, which are highly expressed in various epithelial tissues; VE-cadherins, which are limited in endothelial tissues; N-cadherins, which are abundant in nerve, muscle, lens, as well as fibroblasts; and P-cadherins, which are present in placenta and epidermis (Lodish et al 2004). The nonclassical cadherins include desmosomal cadherins such as desmoglein and desmocollin, protocadherins, and atypical cadherins (Lodish et al 2004).

Selectins are another family of cell-cell adhesion molecules and contain single-chain transmembrane glycoproteins that mediate numerous $\mathrm{Ca} 2+$ dependent cell-cell interactions found within the blood stream. Selectins play a very important role, with the aid of integrins, in the binding of white blood cell to endothelial cells during inflammation (Lodish et al 2004).

The immunoglobulin superfamily (IgSF) of proteins is a large group of cell surface and soluble proteins that are involved in the recognition, binding, or adhesion processes of cells. Members of the IgSF are characterized based upon shared immunoglobulin domains or folds (Lodish et al 2004). Members of the IgSF include cell surface antigen receptors, co-receptors, and co-stimulatory molecules of the immune system, molecules involved in antigen presentation to lymphocytes, cell adhesion molecules, certain cytokine receptors and intracellular muscle proteins. For cell-cell adhesion, IgSF proteins such as N-CAM mediate homotypic cell-cell adhesion, while IgSF proteins such as ICAM and VCAM engage heterotypic cell-cell adhesion (Lodish et 2004).

Cell-to-cell adhesion can form the cell junctional structures that can be visualized under electron microscope. These junctional structures include adherence junction, desmosome, tight junction, and gap junction. Cadherins are the cell-cell adhesion transmembrane proteins that determine the formation of adherence junction and desmosome in a calcium-dependent manner. Cadherins also plays key roles in regulating collective cell movement and tumor metastasis (Canal et al 2010; Serrels et al 2009). For example, E-cadherin is a well accepted tumor metastasis suppressor (Berx et al 1998; Birchmeier and Behrens 1994; Jeanes et al 2008; Perrais et al 2007; Serrels et al 2009; Thiery et al 2002). 


\subsection{TETRASPANINS AND TETRASPANIN-ENRICHED MICRODOMAINS (TEMS)}

\subsubsection{Tetraspanins}

Tetraspanins are a superfamily of transmembrane proteins containing 4 membrane-spanning segments. Tetraspanin proteins regulate cell morphology, cell adhesion, cell motility, cell fusion, and signaling in various organs and tissues such as brain, immune system, and cardiovascular system under physiological conditions (Berditchevski 2001; Helmer 2003; Miranti 2008; Pols 2008; Sridhar 2006). Tetraspanins also regulate these cellular and molecular events during pathological conditions such as tumors, viral infection, and inflammation.

To qualify for membership in the tetraspanin superfamily, a protein must have four transmembrane domains and several conserved amino acids, including an absolutely conserved $\mathrm{CCG}$ motif and two other cysteine residues that contribute to two crucial disulphide bonds within the second extracellular loop (Berditchevski 2007; Liu and Zhang 2006; Miranti 2009; Richardson 2011; Tonoli 2005; Yang et al 2001; Yunta 2003). Of the 200-350 amino acids that are found in tetraspanins, 13-31 of them reside in the short extracellular loop (EC1), for which the structural information is not yet available. The large extracellular loop (EC2, 69-132 amino acids) is subdivided into a constant region and a variable region, which contains nearly all of the known protein-protein interaction sites in tetraspanins. The first, third and fourth transmembrane domains often contain polar residues, which play important roles in 1) maintaining the structural stability of tetraspanins and 2) interacting with other tetraspanins and transmembrane proteins (Bari et al 2009). Almost all tetraspanins are modified by the post-translational addition of palmitate to membrane-proximal cysteine residues. In fact, of the 33 mammalian family members, 30 contain at least one and as many as seven membrane-proximal palmitoylation sites. Tetraspanin proteins also undergo $\mathrm{N}$-glycosylation within its large extracellular loop.

\subsubsection{Tetraspanin-enriched microdomain}

Tetraspanins tend to associate with each other to form homodimers and heterodimers. On the bases of these dimers, tetraspanins further associate to assemble into multimeric transmembrane complex (Berditchevski 2007; Pols 2008; Richardson 2011; Yunta 2003; Zhou et al 2004). For example, tetraspanin CD82 associates with tetraspanins CD9, CD81, CD63, and CD151 (Liu and Zhang 2006; Miranti 2009; Richardson 2011). 
In addition, tetraspanins physically associate with other transmembrane proteins such as cell-cell and cell-matrix adhesion molecules, growth factor receptors, and proteases and organize these transmembrane proteins into membrane complexes termed as tetraspanin webs, glycosynapses, or tetraspanin-enriched microdomains (TEMs), with which intracellular signaling proteins such as PI4K, PKC, and syntenin are associated (Berditchevski 2001; Berditchevski and Odintsova 2007; Helmer 2003; Liu and Zhang 2006; Richardson 2011). The cell adhesion molecules present in TEMs include integrins, IgSF proteins, and cadherins (Liu and Zhang 2006; Miranti 2009; Richardson 2011). However, the biochemical and biophysical mechanisms by which tetraspanins form TEMs and regulate the activities of their associated transmembrane proteins remain largely unclear.

\subsection{THE INTRODUCTION OF TETRASPANIN CD82}

CD82 is a member of the tetraspanin superfamily, was initially discovered as a modulator for immune functions, and was then identified from metastatic rat prostate cancer cells as a suppressor of prostate cancer metastasis (Adachi et al 1996; Dong et al 2005; Liu and Zhang 2006; Protzel 2008; Sridhar 2006; Yang et al 2001). CD82 has now been deemed as a metastasis suppressor for a wide variety of solid tumors (Abe et al 2008; Adachi et al 1996; Dong et al 1996; Dong et al 1997; He at al 2005; Jee et al 2007; Liu and Zhang 2006; Miranti 2008; Protzel et al 2008; Sridhard and Miranti 2006; Tonoli and Barrett 2005; Yang et al 2001). The mechanism that governs CD82-mediated suppression of tumor metastasis, however, still remains largely unknown.

\subsubsection{The chromosomal locus of the CD82 gene}

The $C D 82$ gene is located in the p11.2 region of the human chromosome. The gene spans $\sim 8 \mathrm{~Kb}$ of genomic DNA and consists of 10 exons. The intron-exon organization of the $C D 82$ gene is depicted in Figure 1-1. The CD82 gene contains 9 introns and 10 exons.

\subsubsection{The expression and regulation of the CD82 gene}

The promoter is 795 base pairs. The coding region starts at base 25 of exon 3 , spans exons 4-9 and into the first 75 base pairs of exon 10. The remainder of exon 10 constitutes the 3' untranslated region of CD82 as well as an Alu element. The promoter 


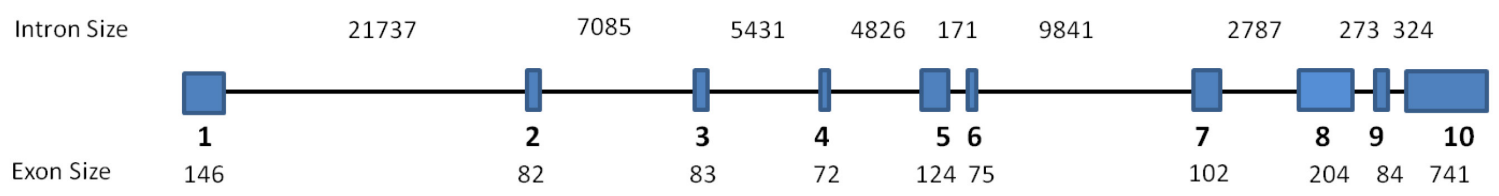

Figure 1-1. The genomic organization of the CD82 gene. There are 10 exons and 9 introns within the $\sim 8 \mathrm{~Kb}$ CD 82 gene.

region of $C D 82$ is $\mathrm{CG}$ rich, contains no TATA box, and has nine binding sites for the Sp1 transcription factor and five binding sites for AP-2 (Dong et al. 1997). In addition to the wild type, the human $C D 82$ gene produces two alternatively spliced variants. The variants, one and two, contain 9 exons, exon 6 or 8 have been deleted, respectively.

\subsubsection{The protein structure of CD82}

The CD82 protein is a 267 amino acid protein with a molecular weight of 30-90 $\mathrm{kDa}$, which is tissue specific and depends upon post-translational modification. CD82, being a member of the tetraspanin superfamily, contains 4 hydrophobic transmembrane domains, a large and a small extracellular loop, and two intracellular cytoplasmic tails, Figure 1-2. For the human CD82 proteins, each of the four transmembrane domains are formed by a hydrophic segment containing, 21,19,27,22, amino acid residues (Dong et al 1997). The hydrophilic extracellular domains/loops 1 and 2 are composed of 21 (positions 33-53) and 118 (positions 111-228) amino acids, respectively (Dong et al 1997). The extracellular domain/loop 2 contains 6 cysteine residues, two of which are located in the conserved CCG motif (Dong et al 1997). The cysteine residues are predicted to form disulphide bonds and are important for proper protein folding and biological functions. Three putative glycosylation sites are located at residue/position 129, 157, and 198, respectively (Dong et al 1997).

The cytoplasmic tails of CD82 protein is short and contains 11 (positions 1-11) and 17 positions (251-267) amino acid residues, respectively (Dong et al 1997). Like other tetraspanins, CD82 is palmitoylated at the five cytoplasmic cysteine residues that are located proximal to the inner leaflet of the plasma membrane at residue/position 5, 74, 83, 251, 253, respectively (Dong et al 1997). At the C-terminal cytoplasmic domain, there is a YSKV sequence, which falls into the category of the YXXФ motif (Y: tyrosine residue, $\mathrm{X}$ : any amino acid residue, and $\Phi$ : hydrophobic amino acid residue with large side chains such as valine, leucine, and isoleucine (Dong et al 1997). 


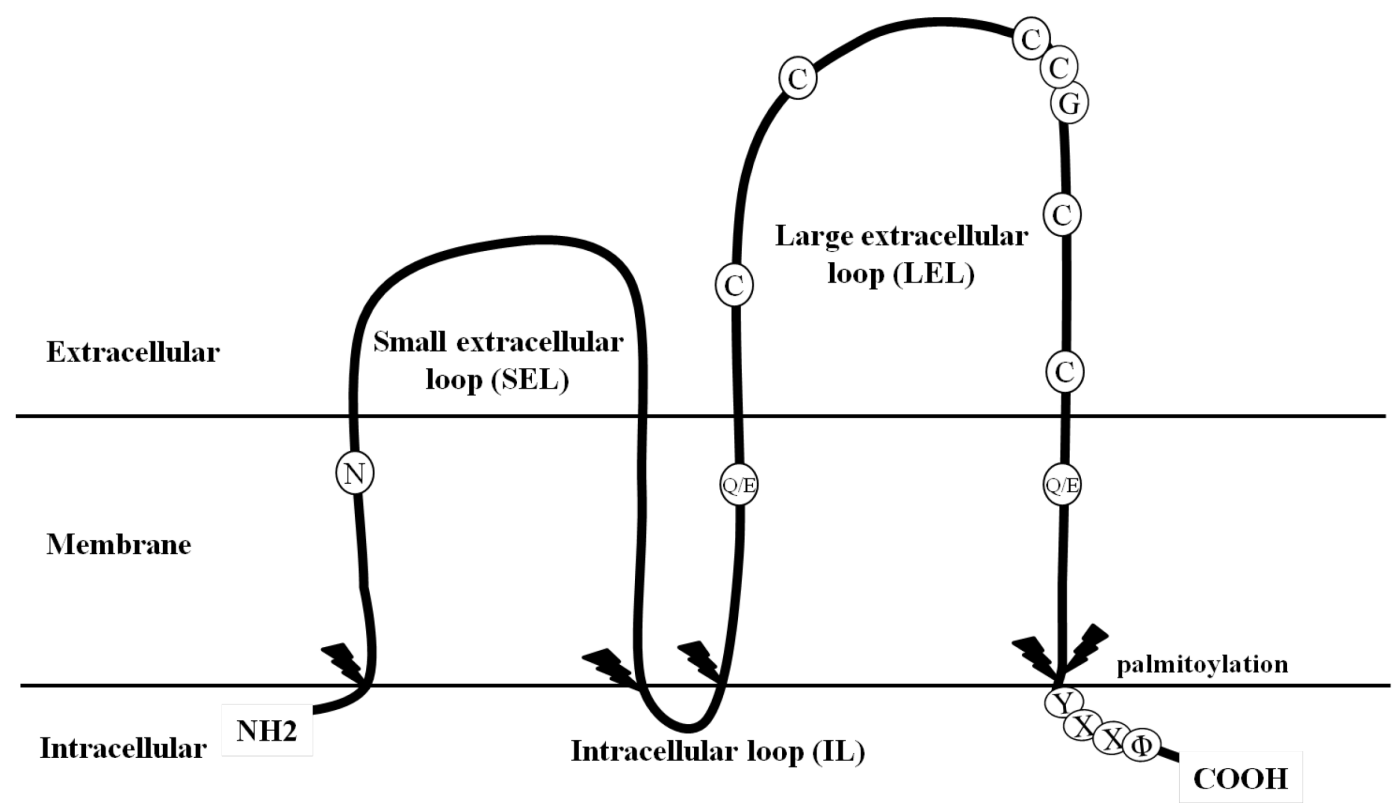

Figure 1-2. The protein structure of the tetraspanin CD82. CD82 contains 4 hydrophobic transmembrane domains, a large and a small extracellular loop, a small intracellular loop and two intracellular cytoplasmic tails. Located within the Cterminal cytoplasmic tail of CD82 is a YSKV sequence that serves as a tyrosine based sorting motif. The large extracellular loop contains 6 cysteine residues, two of which are located in the conserved CCG motif which aids in proper protein folding and several sites for post-translational modification, three putative glycosylation sites and five proximal potential palmitoylation sites. 


\subsubsection{Tissue distribution of CD82}

CD82 is ubiquitously expressed in a wide variety of epithelial cells and is also expressed in endothelial cells (He et al 2005; Liu and Zhang 2006; Miranti 2009; Richardson 2011; Tonoli and Barrett 2005). Northern blot analysis revealed high expression of CD82 in the spleen, thymus, prostate, ovary, small intestine, colon, placenta, lung, liver, kidney, and pancreas, relatively lower expression in the heart, brain, muscle, and testis (Liu and Zhang 2006; Miranti 2009). In mice, CD82 was found in all tissue except the arterioles and brain (Miranti 2009).

\subsection{THE PATHOLOGICAL FUNCTIONS OF CD82: CD82 AND TUMOR PROGRESSION AND METASTASIS}

CD82 has been well documented to correlate with patient prognosis in various solid malignant tumors such as lung, pancreatic, breast, bladder, colon, esophageal, cervical, and endometrial cancers (Adachi et al 1996; Miranti 2009; Richardson 2011; Yang et al 2001; Uchida 1999). The tumor patients whose tumors express CD82 have a significantly greater chance of survival or longer survival time than those whose tumors have a decrease in or loss of CD82 expression (Liu and Zhang 2006; Marinti 2009; Tonoli and Barrett 2005).

Inverse correlation of CD82 expression and metastasis has been observed in breast (Christgen 2008; Christgen 2009; Higashiyama et al 1998; Huang et al 1998), prostate (Bouras et al 1999; Dong et al 1996), liver (Guo et al 1998; Sun et al 1998), colon (Lombardi et al 1999; Maurer et al 1999; Wu et al 2004), stomach (Hionda 1998; Wu 2003;), pancreas (Friess et al 2001; Guo et al 1996), oral (Farhadieh et al 2004; Geradts et al 1999; Imai et al 2002; Uzawa et al 2002), ovary (Liu et al 2000; Schindl 2008), endometrium (Briese 2008; Li et al 2011; Liu et al 2003; White et al 1998;), lung (Adachi et al 1996; Higashiyama 1998) thyroid (Chen et al 2004), SCC of the head and neck (Xiong et al 2005) esophagus (Miyazaki et al 2000), penile (Protzel et al 2008), and urothelial bladder cancers (Su et al 2004; Yu et al 1997).

For example, CD82 is highly expressed in normal mammary epithelium, benign mammary gland tumor, and early malignant ductal carcinoma in situ (DCIs) lesions of breast cancer. Invasive ductal breast cancer and brain metastasis showed that CD82 mRNA expression was significantly lower when compared to benign mammary gland tumors (Christgen et al 2008; Christgen et al 2009). In breast cancers, CD82 expression is correlated with estrogen receptor expression (Christgen et al 2008; Christgen et al 2009). CD82 expression is down regulated in ER-positive breast cancer cells and highly expressed in ER-negative breast cancer cells (Christgen et al 2009). 
In prostate cancer, CD82 expression correlated with disease progression and disease free survival. $90 \%$ of patients who did not respond to androgen ablation therapy exhibited tumors with a loss of CD82 expression (Dong et al 1996). When CD82 expression was restored in a highly metastatic rat prostate carcinoma cell line, the metastasis of prostate cancer was markedly suppressed (Dong et al 1995; Dong et al 1996).

The studies on other solid malignant tumors reached similar conclusions. In hepatocellular carcinoma, CD82 is down regulated at both the mRNA and protein levels (Guo et al 1998). CD82 expression correlates with intrahepatic metastasis but not with tumor size or expression of $\alpha$-fetoprotein (Guo et al 1998; Sun et al 1998). In colorectal carcinoma, CD82 was down regulated upon the disease progression (Wu et al 2004). In gastric cancer, CD82 expression is inversely correlated with disease progression (Hinoda 1998; Wu et al 2003). Down regulation of CD82 is frequently observed in ovarian cancer (Liu et al 2000; Schindl 2008).

In Non Small Cell lung cancer, CD82 expression is inversely correlated with the disease progression and is therefore a good prognostic factor (Adachi et al 1996; Higashiyama et al 1998). Down regulation of CD82 in thyroid cancer is inversely correlated with patient prognosis (Chen et al 2004). CD82 expression is correlated with squamous cell carcinoma (SCC) of the head neck, lung, and cervix (Xiong et al 2005). In SCC, CD82 expression is not correlated with allelic loss, promoter hyper-methylation, gene mutation, or $\mathrm{p} 53$ regulation but was correlated with lymph node metastasis, disease-free survival and overall survival (Imai et al 2002; Xiong et al 2005). CD82 expression can be used as a marker in penile and bladder cancer but not cervical cancer (Protzel et al 2008, Su et al 2004). Patients whose tumors exhibit CD82 have a significantly greater chance of survival than those whose tumors have a decrease in or loss of CD82 expression (Abe et al 2008; Protzel et al 2008; Sridhar and Miranti 2006; Yang et al 2001). CD82 has also been re-expressed in a highly metastatic prostate rat carcinoma cell line and was able to inhibit metastasis (Miranti 2009; Yang et al 2001).

\subsection{CD82-CONTAINING TETRASPANIN-ENRICHED MICRODOMAIN}

The mechanism by which CD82 inhibits metastasis has not been entirely established. It is believed that CD82 suppressive function is mediated by the association of other proteins. CD82 as well as other proteins are known to bind together and form tetraspanin enriched microdomains (Berditchevski 2001; Berditchevski and Odintsova 2007; Helmer 2003; Liu and Zhang 2006; Richardson 2011). In fact, CD82 has been reported to be important for regulating cell signaling, cell adhesion, and sorting/trafficking of proteins to the cell surface (He et al 2005; Jee et al 2007; Liu and 
Zhang 2006; Miranti 2009; Tonoli, 2005; Zhou et al 2004). One of the suggested mechanisms is that CD82 functions through its associations with integrins and other tetraspanins (Pols and Klumperman 2008; Zhou et al 2004). CD82 has been shown to associate with $\alpha 2 \beta 1, \alpha 3 \beta 1, \alpha 4 \beta 1, \alpha 5 \beta 1, \alpha 6 \beta 1$, and $\alpha \mathrm{L} \beta 2$ (Abe et al 2008; He et al 2005; Jee et al 2007; Liu and Zhang 2006; Miranti 2009; Sridhar 2006; Sridhar and Miranti 2006; Tonoli and Barrett 2005). In Du145 prostate cancer cells, using function blocking antibodies, it was discovered that $\alpha 6 \beta 1$ integrin is responsible for the CD82-depenent cellular morphogenesis (He et al 2005).In another study, CD82 re-expression in a rat metastatic cell resulted in the reduction of $\alpha 6$ levels, as well as a reduction in cell adhesion. However in this study, it was observed that there was an increase in $\alpha 6$ adhesion. However in this study, it was observed that there was an increase in $\alpha 6$ internalization (Miranti 2009; Sridhar and Miranti 2006).

CD82 has also been shown to associate with other tetraspanins such as CD9, CD81, CD63, CD151, and EWI-2 (Liu and Zhang 2006; Miranti 2009; Sridhar and Miranti 2006). Morever, growth factor receptors such as EGF receptor and HGF receptor c-Met and IgSF proteins such as EWI-2 were reported to associate with CD82. All of these tetraspanins and other CD82-associated proteins play key roles in the regulation of cell motility.

\subsection{CD82 AND CELL-CELL ADHESION}

CD82 may also function as a tumor suppressor through cell adhesion molecules, CAMs. CAMs mediate direct cell-cell adhesions (Abe et al 2008; Lodish 2004; Weinberg 2007).The major cell-cell adhesion molecule for epithelial cells is epithelial cadherins or E-cadherins. E-cadherins forms adherence junctions with each other and act as mechanical linkages between cells. E-cadherin is thought to play a role in preventing tumor metastasis because it is commonly found to be down regulated in metastatic carcinomas (Abe et al 2008; Miranti, 2009). CD82 is located near adherens junctions which may facilitate its role in adhesion strengthening (Abe et al 2008). Abe et al reported that CD82 regulates E-cadherin-dependent cell adhesion by preventing dissemination in h1299 cells.

\subsection{THE TRAFFICKING OF CD82}

Besides plasma membrane, CD82 is also present in intracellular vesicles (Escola et al 1998; Hammond et al 1998). A recent study from our lab demonstrated that 1) CD82 undergoes endocytosis and traffics to endosomes and lysosomes, 2) dynamin and clathrin are not essential for CD82 internalization, and 3) depletion or sequestration of sterol in 
the plasma membrane markedly inhibited the endocytosis of CD82 (Xu et al 2009). In primary dendrtic cells, CD82 is recruited to $\mathrm{C}$. neoformans-containing phagosomes in a phagosome acidification-independent manner or prior to the step of lysosomal fusion (Artavanis-Tsakonas et al 2011).

Interestingly, CD82 and other tetraspanins are highly enriched in exosomes (Escola et al 1998), suggesting the putative roles of CD82 in exosome formation and release. Notably, Chairoungdua et al recently showed that CD82 suppresses beta catenin-mediated Wnt signaling by inducing the exosomal release of beta catenin (Chairoungdua et al 2010). 


\section{CHAPTER 2. REGULATION OF TRAFFICKING BY THE YXXФ MOTIF OF CD82}

\subsection{INTRODUCTION}

Some tetraspanins such as CD9 and CD81 are exclusively expressed on the cell surface whereas others such as CD82 contain a large intracellular pool, in addition to that found on the plasma membrane. Immuofluorescent studies revealed that CD82 proteins are present at the cell peripheries and intracellular vesicles (Richardson 2011; Xu et al 2010). Electron microscopic and proteomics studies showed that CD82 proteins are also enriched in multi-vesicular bodies (MVBs) and exsosomes (Miranti, 2009; Richardson 2011). These observations strongly argue for the active trafficking of CD82 proteins 1) between the plasma membrane and endosomal/lysosomal compartments and 2) extracellular environment.

Indeed, an earlier study from our lab demonstrated that CD82 undergoes endocytosis and the internalized CD82 traffics to late endosomes and lysosomes (Xu et al 2009). Interestingly, dynamin and clathrin are not essential for CD82 internalization (Xu et al 2009), suggesting that CD82 is internalized through a clathrin-independent mechanism. The perturbation, depletion, or sequestration of cholesterol in the plasma membrane markedly inhibited the endocytosis of CD82 (Xu et al 2009), suggesting that CD82 endocytosis depends on cholesterol. The study also found that CD82 endocytosis is distinct from macropinocytosis and other dynamin-independent pinocytosis ( $\mathrm{Xu}$ et al 2009). Functionally, the lipid-dependent endocytosis of CD82 appears to alleviate CD82-mediated inhibition of cell migration (Xu et al 2009).

Notably, CD82 contains an YSKV sequence in its C-terminal cytoplasmic domain. This YSKV sequence falls into the category of the YXXФ sorting motif, in which $\mathrm{Y}$ is a tyrosine residue, $\mathrm{X}$ can be any amino acid residue, and $\Phi$ represents bulky hydrophobic amino acid residues such as L, I, and V (Marks et al 2007). As described above in clathrin-dependent endocytosis, the cytoplasmic domains of transmembrane molecules usually contain conserved internalization motifs such as the YXXФ sequence. Since CD82 endocytosis appears to be independent of the YXXФ motif, whether this motif plays roles in the targeting CD82 to endosomal and lysosomal compartments or to exosomes remains to be determined. More importantly, whether this motif plays a role in the function of CD82, such as the inhibition of cell movement needs to be assessed.

To determine whether the YSKV sequence of CD82 is involved in the regulation of its intracellular trafficking and biological functions, we mutated the tyrosine based sorting motif of CD82. After establishing the stable transfectants of this mutant in PC3 
and Du145 human metastatic prostate cancer cells, we examined the role of CD82 YXXФ motif in the endocytosis, recycling, lysosomal targeting, and exosomal release of CD82. We also investigated the roles of CD82 YXXФ motif in the surface expression and trafficking of CD82-associated proteins as well as the cell adhesion proteins important for cell movement.

Our studies suggest that the tyrosine-based sorting motif of CD82 is not important for the internalization of CD82 proteins but regulates the intracellular trafficking such as lysosomal targeting, recycling, and exosomal release of CD82 proteins.

\subsection{MATERIALS AND METHODS}

\subsubsection{Materials}

The monoclonal antibodies (mAbs) used in this study were human CD82 mAb TS82b (kindly provided by Dr. Eric Rubenstein), CD63 mAb (Ancell), EEA-1 mAb (BD Biosciences), LBPA mAb (Echelon), murine IgG2b (Sigma), and integrin $\beta 1 \mathrm{mAb}$ TS2/16. Fluorescent reagents include FITC-conjugated goat-anti-mouse IgG (Sigma), fluorescent probes Alexa-488 and Alexa-594 (Invitrogen), and LysoTracekr Red DND99 (Invitrogen). Other reagents include detergents Brij 97 and 98 (Sigma), NP-40 (Fluka), ultracentrifuge tubes (Beckman), lipofectanine 2000 (Invitrogen), Geneticin or G418 (Gibco/Life Technology), Chemiluminesence reagents (Perkin Elmer), goat serum (Gibco/Life Technology), DMEM (Gibco/Life Technology), x-ray film (Perkin Elmer), and paraformaldehyde (Fisher).

\subsubsection{Cell culture}

PC3 and Du145 human metastatic prostate cancer cells were obtained from ATCC and cultured in DMEM, supplemented with 10\% fetal bovine serum, 100 units $/ \mathrm{ml}$ penicillin, $100 \mu \mathrm{g} / \mathrm{ml}$ streptomycin, and $2 \mathrm{mM} \mathrm{L-glutamine,} \mathrm{at} 37^{\circ}$ in a $5 \% \mathrm{CO} 2$ incubator.

\subsubsection{Cells and transfectants}

The CD82 wild type (WT) and CD82 YVAA mutant constructs were generated by fusing CD82 cDNA with a eukaryotic expression vector (pcDNA 3.1+ or pCRuni). Briefly, the CD82 YVAA mutant was generated by simultaneously replacing the Tyrosine and Valine residues in the YSKV sequence at the C-terminal cytoplasmic 
domain of the wild type CD82 with the Alanine residues through PCR. The full length cDNAs encoding human wild type and YVAA mutant CD82 were constructed into pcDNA $3.1+$ or pcrUNI vectors, respectively, through the XbaI and HindIII sites. The sequences of CD82 coding frame were confirmed by DNA sequencing.

PC3 and DU145 cells were transfected with the plasmid DNA using Lipofectamine 2000 (Invitrogen) and selected under geneticin (g418) at a concentration of 1.0 and $0.5 \mathrm{mg} / \mathrm{ml}$, respectively. The G418-resistant clones were pooled together, and the CD82-positive clones were obtained by flow cytometric cell sorting. The sorted CD82-positive clones were maintained as the CD82 WT or YVAA stable transfectants.

\subsubsection{Western blot}

Cells were lysed with $1 \%$ Brij 97 and/or $1 \%$ Nonidet P-40 lysis buffer at $4{ }^{\circ} \mathrm{C}$ for $1 \mathrm{~h}$. Insoluble material was removed by the centrifugation at $14,000 \times \mathrm{g}$ for $15 \mathrm{~min}$. The cell lysates were mixed with Laemmli sample buffer and heated at $95{ }^{\circ} \mathrm{C}$ for $5 \mathrm{~min}$. The proteins were separated by SDS-PAGE and electrically transferred to nitrocellulose membranes (Bio-Rad). The membranes were sequentially blotted with primary $\mathrm{mAb}$ and horseradish peroxidase-conjugated second $\mathrm{Ab}$ (Sigma), followed by chemiluminescence (Perkin Elmer Life Sciences).

\subsubsection{Flow cytometry}

For flow cytometry, confluent cells were detached, washed with 1xPBS, and blocked with $2 \%$ goat serum on ice for 1 hour to block the non-specific binding. Cells were incubated with $1 \mu \mathrm{g} / \mathrm{ml}$ of primary mAb such as CD $82 \mathrm{mAb}$ TS82b, integrin $\beta 1$ $\mathrm{mAb}$ TS2/16, or murine IgG2b on ice for 1 hour followed by PBS washes. The cells were then labeled with appropriate flurochrome-conjugated secondary Ab on ice for 30 mins followed by extensive PBS washes. The cell surface expressions of the proteins were analyzed using a FACS Calibur flow cyotmeter (BD Biosciences).

\subsubsection{Internalization and recycling assays}

The internalization of CD82 was examined by an antibody uptake assay as previously described (Liu et al 2007). Briefly, adherent cells were incubated with $1 \mu \mathrm{g} / \mathrm{ml}$ of CD $82 \mathrm{mAb}$ TS82b on ice for $1 \mathrm{hr}$. The unbound $\mathrm{mAb}$ was removed by the washes with ice cold media, and the cells were incubated at $37^{\circ}$ in $5 \% \mathrm{CO} 2$ for $1 \mathrm{hr}$ for internalization. The cell surface-bound or un-internalized antibody was removed with acidic washes $(0.1 \mathrm{M}$ glycine buffer, $\mathrm{pH}$ 2.7). The cells were fixed, permeablized, and 
blocked with $20 \%$ goat serum. The cells were labeled with flurochrome-conjugated secondary antibody, extensively washed, mounted with FluroSave solution, and examined with either fluorescent microscope or flow cytometry.

The recycling of CD82 was examined by the antibody uptake followed by the antibody retrieval and removal at the cell surface. Briefly, the cells were incubated with 1 $\mu \mathrm{g} / \mathrm{ml}$ of CD82 $\mathrm{mAb}$ TS82b on ice for $1 \mathrm{hr}$. The unbound $\mathrm{mAb}$ was removed by washes with ice cold media followed by $1 \mathrm{hr}$ incubation at $37^{\circ}$. The cell surface-bound, uninternalized $\mathrm{mAb}$ was removed with acidic washes (0.1 M glycine buffer, $\mathrm{pH} 2.7)$, and then the cells were re-incubated with medium at $37^{\circ}$ for 1 or $3 \mathrm{~h}$ to allow the recycling of the internalized $\mathrm{mAb}$. The cells were acid washed, fixed, permeablized, and blocked for non-specific binding with $20 \%$ goat serum. The cells were then labeled with Alexa 594-conjugated secondary antibody, extensively washed, and then either mounted with a FluroSave solution for microscopic analysis or examined directly using flow cytometry.

\subsubsection{Intracellular trafficking of CD82}

The intracellular tracking of CD82 proteins to endosomal and lysosomal compartments was assessed by analyzing the co-distribution of CD82 with the intracellular organelle markers at the steady state.

For the co-localization of CD82 with CD63, the cells were fixed, permeablized, blocked, and then incubated with FITC-conjugated CD63 mAb and Alexa 594-conjugated CD $82 \mathrm{mAb}$ TS82b for 1.5 hours, followed by extensive PBS washes, and mounted for microscopic analysis.

For the co-localization of CD82 with LysoTracker, the cells were incubated at $37^{\circ}$ in $5 \% \mathrm{CO}_{2}$ for 10 minutes in the presence of $0.25 \mu \mathrm{g} / \mathrm{ml}$ of LysoTracker Red DND99, followed by rinsing with fresh media. The cells were fixed, permeablized, blocked, and incubated with Alexa 488-conjugated CD82 mAb TS82b for 1.5 hours, extensively washed with PBS, and mounted for microscopic analysis.

For the co-localization of CD82 with lysobiphosphatic acid (LBPA), a double staining procedure was applied. The cells were fixed, permeablized, blocked, and incubated with $1 \mu \mathrm{g} / \mathrm{ml}$ of LBPA followed by labeling with Alexa 594-conjugated secondary antibody for 1 hour and extensive washes. The cells were further blocked with an isotype matched $\mathrm{mAb}$ for 1 hour to saturate the binding sites of the secondary $\mathrm{Ab}$. The cells were then incubated with Alexa 488-conjugated CD82 mAb TS82b for 1.5 hours, extensively washed with PBS, and mounted for microscopic analysis. 
For the co-localization of CD82 with Rab 11 WT or dominant negative mutant, the transfectant cells were transiently transfected with Rab 11 WT-GFP or Rab 11 dominant negative mutant-GFP constructs and grown for 48 hours. The cells were fixed, permeablized, blocked with $20 \%$ goat serum in PBS, incubated with Alexa 594-conjugated CD82 mAb TS82b for 1.5 hours, extensively washed with PBS, and mounted for confocal microscopic analysis as described at following.

For the co-localization of integrins $\alpha 2, \alpha 3, \alpha 5$, and $\alpha 6$, we followed the internalization assay, see section 2.2.6. Briefly, adherent cells were incubated with 1 $\mu \mathrm{g} / \mathrm{ml}$ of integrin $\alpha 2 \mathrm{mAb}$ IIE10, integrin $\alpha 3 \mathrm{mAb} \mathrm{A} 3 \mathrm{X} 8, \alpha 5 \mathrm{mAb}$ BIIG2, and integrin $\alpha 6$ $\mathrm{A} 6 \mathrm{BB} \mathrm{mAb}$ on ice for $1 \mathrm{hr}$. The unbound $\mathrm{mAb}$ was removed by the washes with ice cold media, and the cells were incubated at $37^{\circ}$ in $5 \% \mathrm{CO} 2$ for $1 \mathrm{~h}$ for internalization. The cell surface-bound or un-internalized antibody was removed with acidic washes $(0.1 \mathrm{M}$ glycine buffer, $\mathrm{pH}$ 2.7). The cells were fixed, permeablized, and blocked with $20 \%$ goat serum. The cells were labeled with an Alexa-594-conjugated secondary antibody, extensively washed. The cells were then blocked with an IgG isotype matched $\mathrm{mAB}$ and further probed with a directly conjugated Alexa-488CD82, TS82b, mAb, followed by extensive washing. The cells were then mounted with FluroSave solution, and examined with either fluorescent microscope or flow cytometry.

For the quantification of co-localization, the Ziess LSM software was used to measure the amount of CD82 staining that is co-localized with the markers of endosomal and lysosomal compartments as described above.

\subsubsection{Fluorescent and confocal microscopy}

The cells were fixed, permeablized, blocked, and incubated with Abs and fluorescent probes as described above. For immunofluorescence analysis, the cells were examined with an Axiophot fluorescent microscope (Carl Zeiss) at the magnification of $63 \mathrm{x}$, and the images were captured using an optronics digital camera. For confocal microscopic analysis, cells were examined using a LSM 510 laser scanning microscope (Carl Zeiss) using excitation lasers for the 488 and $594 \mathrm{~nm}$ wavelengths and a 100x/1.4 objective.

\subsubsection{Exosome isolation}

Exosomes were isolated as previously described (Nazarenko et al 2010). Briefly, $10 \times 10^{6} \mathrm{PC} 3$ cells or $20 \times 10^{6}$ Du145 cells were grown overnight in complete DMEM, washed twice with PBS, replenished with incomplete DMEM, and further grown for 48-72 hours. The conditioned medium (or culture supernatant) was subjected to the 
following differential centrifugation steps: 1) $500 \mathrm{xg}$ for 10 minutes twice, 2) 2,000 x g for 20 minutes, 3) 10,000 x g for 30 minutes, and 4) 100,000 x g for 90 minutes. The pellets from last centrifugation were washed with excessive amounts of PBS and centrifuged at 100,000xg for 90 minutes to obtain the purified exosomes.

For protein analysis, $30 \mu 1$ of medium was removed after each of the first 5 centrifugation step and the purified exosomes were resuspended in $100 \mu 1$ of PBS, dissolved in Laemmli sample buffer, heated at $95^{\circ} \mathrm{C}$ for $5 \mathrm{~min}$, separated by SDS-PAGE, and then electrically transferred to nitrocellulose membranes (Bio-Rad). The membranes were sequentially blotted with primary $\mathrm{mAb}$ and horseradish peroxidase-conjugated second mAb (Sigma), followed by chemiluminescence (Perkin Elmer Life Sciences).

\subsection{RESULTS}

\subsubsection{Generation of stable CD82 WT and CD82 YVAA transfectants in PC3 and Du145 cells}

We used a PCR-based, site-directed mutagenesis approach to mutate the tyrosine-based internalization/sorting motif at the CD82 C-terminal domain from the YSKV sequence to the ASKA sequence, referred to as YVAA mutant hereafter. Using lipofectamine-based transfection method, we generated MOCK, CD82 WT and CD82 YVAA stable transfectants in PC3 and Du145 human metastatic prostate cancer cells. Compared to the PC3-MOCK cells, PC3-CD82 WT and-CD82 YVAA transfectants expressed significantly more CD82 proteins both at the cell surface and in total cells. PC3-CD82 WT and -CD82 YVAA transfectants express equivalent amounts of total cellular CD82 proteins, as analyzed by Western blot, as shown in Figure 2-1, and similar levels of CD82 at the cell surface, as measured by flow cytometry, as shown in

Figure 2-2.

\subsubsection{Mutation of the YXXФ motif of CD82 did not alter the steady state distribution of $\mathrm{CD82}$}

The subcellular distribution and endosomal and lysosomal localization of CD82 likely plays a role in its motility-suppressive function. We analyzed the steady state localization of CD82 WT and CD82 YVAA mutant. The immunofluorescence analysis revealed that the mutation in the internalization/sorting motif of CD82 did not alter the subcellular distribution of CD82 proteins, when comparing the immunofluorescence staining of CD82 WT and CD82 YVAA in either non-confluent or confluent cell growth stages, as shown in Figure 2-3. 


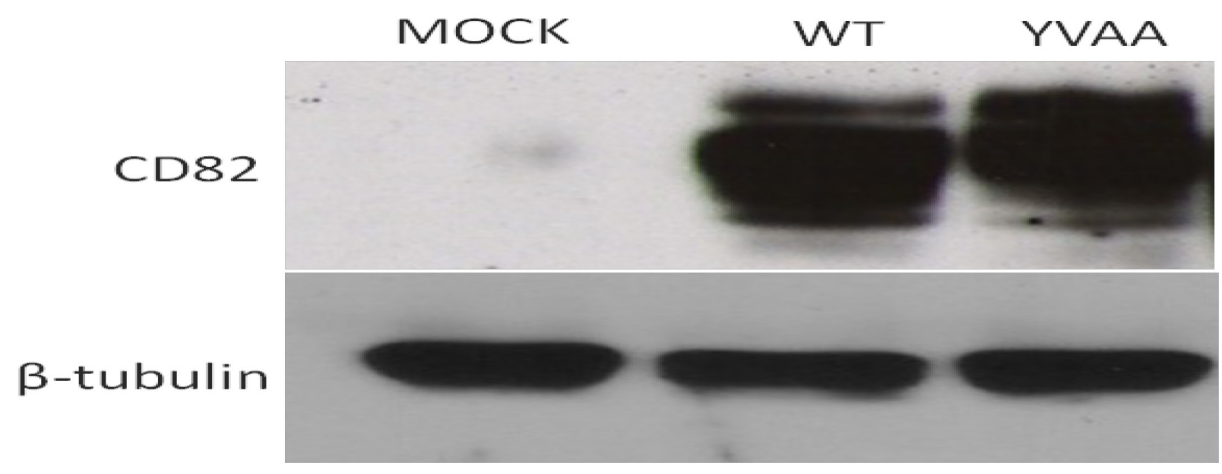

Figure 2-1. The expression of CD82 proteins in PC3 transfectant cells were analyzed by Western blot. PC3-Mock, -CD82 WT, and -CD82 YVAA cells were detached and lysed in 1\% NP-40 lysis buffer. Equal amounts of cell lysate were resolved by SDS-PAGE. Proteins were transferred to nitrocellulose membrane and then probed with CD82 mAb TS82b and $\beta$ tubulin mAb. $\beta$ tubulin served as a loading control. 


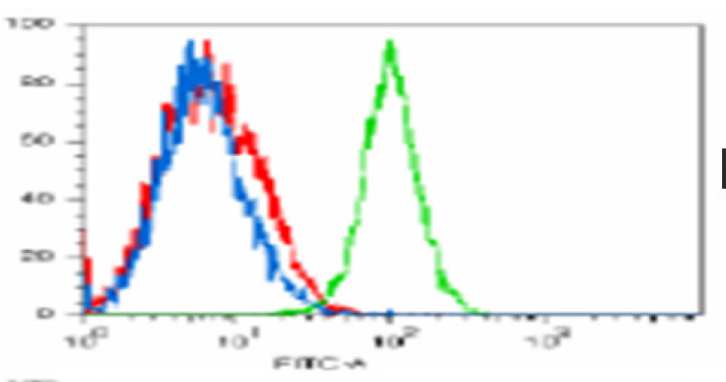

MOCK

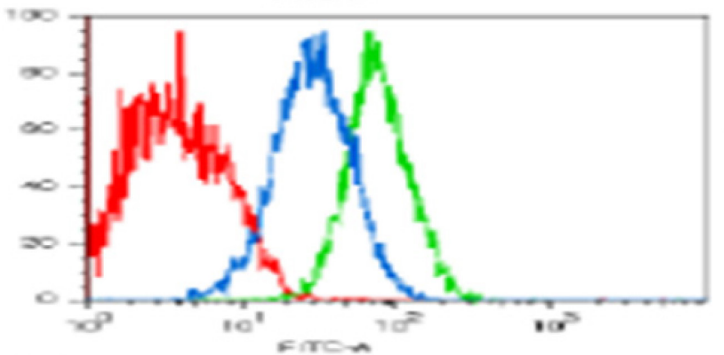

WT

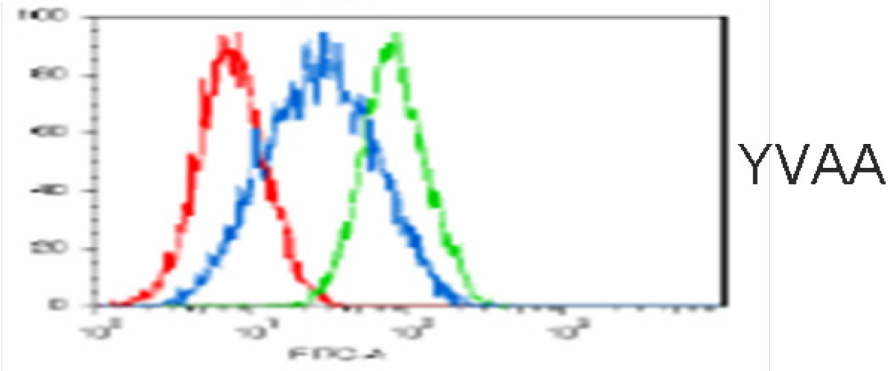

Figure 2-2. The expression of CD82 proteins at the surface of PC3 transfectant cells were analyzed by flow cytometry. PC3-MOCK, -CD82 WT, and-CD82 YVAA transfectant cells were detached, incubated with a negative control mAb (murine IGg2b), a CD82 mAb (TS82b), and a positive control mAb ( $\beta 1$ integrin mAb $\mathrm{TS} 2 / 16$ ) and then analyzed by flow cytometry after a FITC-conjugated second $\mathrm{Ab}$ incubation. The mean fluorescence intensity of CD82 staining for PC3-MOCK, PC3-CD82 WT, and PC3-CD82 YVAA transfectant cells was 243, 1710, and 1578, respectively. 


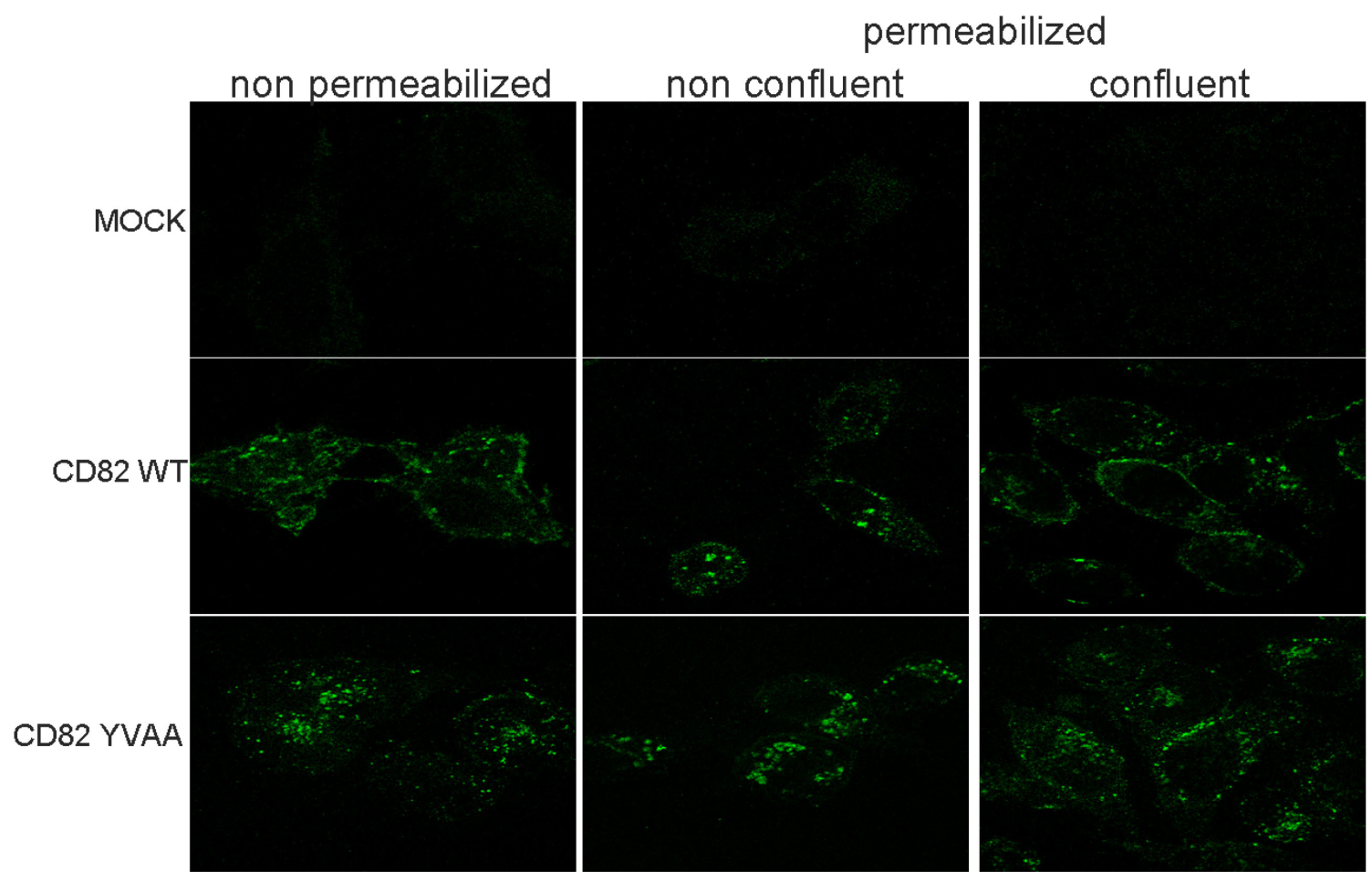

Figure 2-3. The mutation of the YXXФ motif of CD82 does not result in an alteration of the steady state distribution of $\mathrm{CD82}$ proteins in $\mathrm{PC3}$ cells. PC3 -MOCK, -CD82 WT, and -CD82 YVAA transfectant cells were fixed, permeablized, blocked for non-specific binding, and then incubated with the Alexa 488 directly-conjugated CD82 mAb (TS82b). The cells were extensively washed and mounted. Images were captured by using a fluorescent microscope equipped with a digital camera. 


\subsubsection{Mutation of the YXXФ motif of CD82 does not abrogate CD82 internalization}

The internalization and subsequent trafficking of transmembrane proteins regulate their function. CD82 has been shown to alter the function of the protein that are sequestered into TEMs and has also been shown to prevent the retraction of cells during cell migration. The YXX $\Phi$ motif is a determinant for the endocytosis of many transmembrane proteins. The role of this motif in CD82 endocytosis has not been experimentally addressed.

As shown in Figure 2-4, the mutation of the YXXФ motif of CD82 did not inhibit the internalization of CD82 and could even lead to a slight increase in CD82 internalization. The quantification of CD82 endocytosis assayed by either immunofluorescence or flow cytometry came to the same conclusion, Figure 2-5. In Figure 2-5A, the average numbers of CD82-positive, intracellular vesicles after 1-hr endocytosis assayed by immunofluorescence is presented. Figure 2-5B depicts the mean fluorescence intensity of the intracellular CD82 staining after $1 \mathrm{hr}$ endocytosis.

\subsubsection{The mutation of the YXXФ motif of CD82 alters CD82 recycling}

Since the YXXФ motif of CD82 is not responsible for regulating the internalization of CD82, it may regulate the trafficking of CD82 back to the plasma membrane or the recycling process of CD82. The results from the immunofluorescence based recycling assay revealed that 1) there is a slow pace in recycling of CD82 proteins and 2) there is an accumulation of the CD82 YVAA mutant proteins within the intracellular compartments in a time-dependent manner, as shown in Figure 2-6.

The images presented in Figure 2-6 shows that similar numbers of CD82-positive intracellular vesicles can be found between PC3-CD82 WT and-CD82 YVAA cells after 1 hour recycling. However, in contrast to CD82 WT-positive intracellular vesicles, there is an increase in the number of CD82 YVAA-positive vesicles within the cells after an additional 2 hours of recycling.

The quantitative data presented in Figure 2-7 represents the average levels of internalized CD82 proteins that were still remained inside PC3 transfectant cell after 1 and $3 \mathrm{hr}$ recycling. In Figure 2-7A, the quantitative data of recycling were obtained from the immunofluorescence images. We also measured the CD82 recycling using the flow cytometry-based recycling assay. The histogram in Figure 2-7B represents the mean fluorescent intensity (MFI) of the CD82 proteins staining that remained intracellularly after the given periods of recycling. The data obtained from the flow cytometry based recycling assay are quite consistent with the results obtained from the 


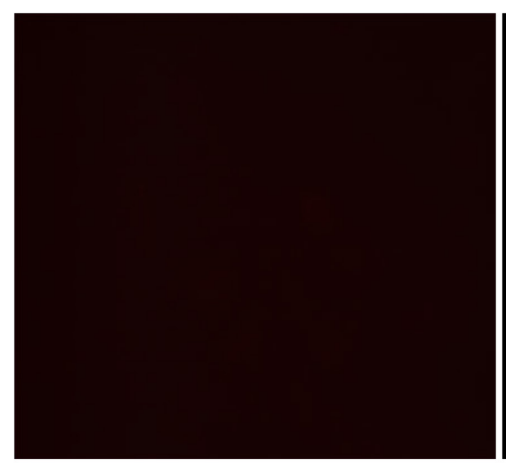

CD82 MOCK

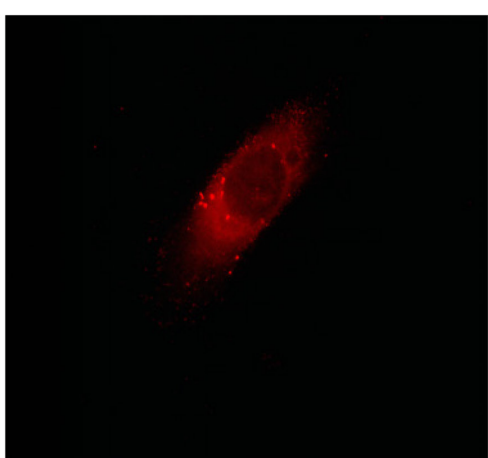

CD82 WT

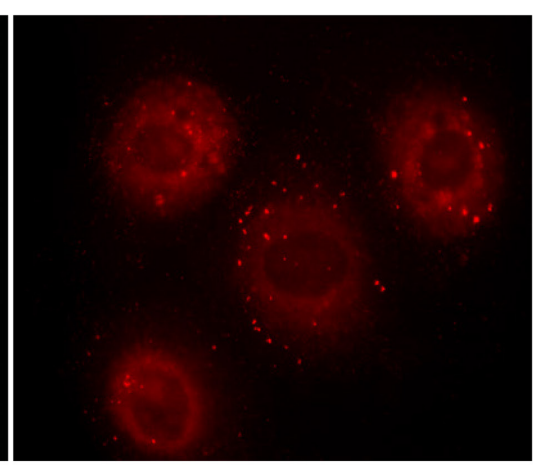

CD82 YVAA

Figure 2-4. The mutation of the YXXФ motif of CD82 does not significantly reduce CD82 internalization. The PC3-Mock, -CD82 WT, and -CD82 YVAA transfectant cells were plated onto glass coverslips and grown overnight in complete media. The cells were incubated with CD $82 \mathrm{mAb}$ TS $82 \mathrm{~b}$ at $4^{\circ} \mathrm{C}$ for $1 \mathrm{~h}$. The cells were then allowed to internalize the bound $\mathrm{mAb}$ by the incubation at $37^{\circ} \mathrm{C}$ for $1 \mathrm{~h}$, followed by acid washes to remove un-internalized mAbs. The cells were fixed, permeablized, and incubated with Alexa 594-conjugated 2nd mAb. The cells were then analyzed using a fluorescent microscope. 
A

Internalization Assay single cell quantification of $\mathbf{F}$

figures

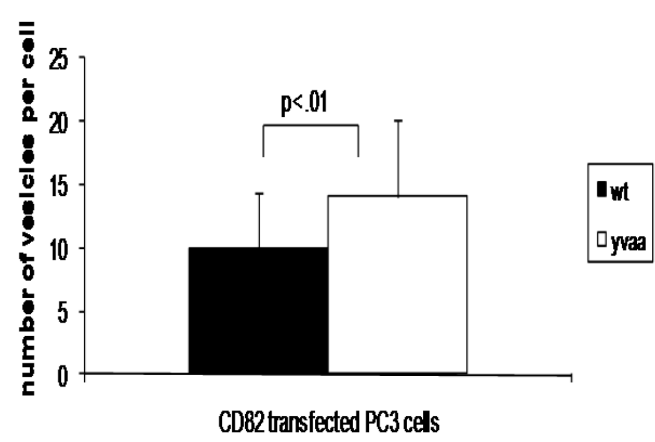

B

Internalization Assay using FACS analysis

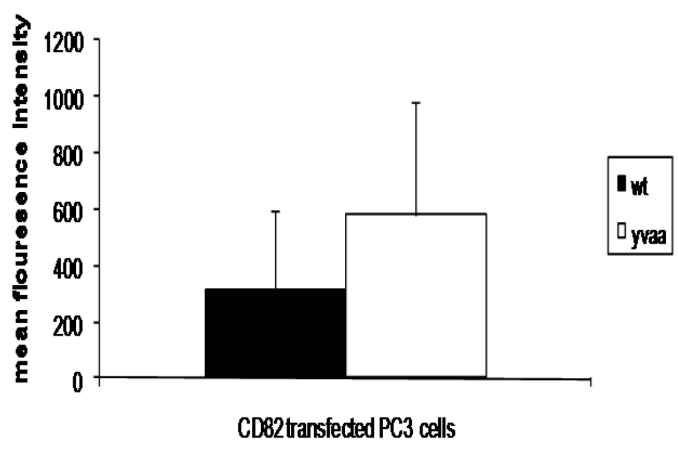

Figure 2-5. The mutation of the YXXФ motif of CD82 does not reduce but may enhance CD82 internalization. PC3-MOCK, -CD82 WT, and -CD82 YVAA transfectant cells were plated onto glass coverslips and grown overnight. Cells were incubated with CD82 $\mathrm{mAb}$ TS82b at $4^{\circ} \mathrm{C}$ for $1 \mathrm{~h}$. Cells were then allowed to internalize the bound $\mathrm{mAb}$ at $37^{\circ} \mathrm{C}$ for $1 \mathrm{~h}$, acid-washed, fixed, permeabilized, and incubated with a Alexa 594-labeled secondary antibody. A, the endocytosis of CD82 was assessed by immunofluorescence using a fluorescent microscope; the quantification was performed by counting the number of CD82-positive intracellular vesicles per cell. In each experiment, 50 CD82-positive cells were quantified per transfectant. B, the endocytosis of CD82 was assessed for the mean fluorescence intensity of the vesicles inside the cell by flow cytometry. The histograms represent the average and standard deviation of 4 experiments. 


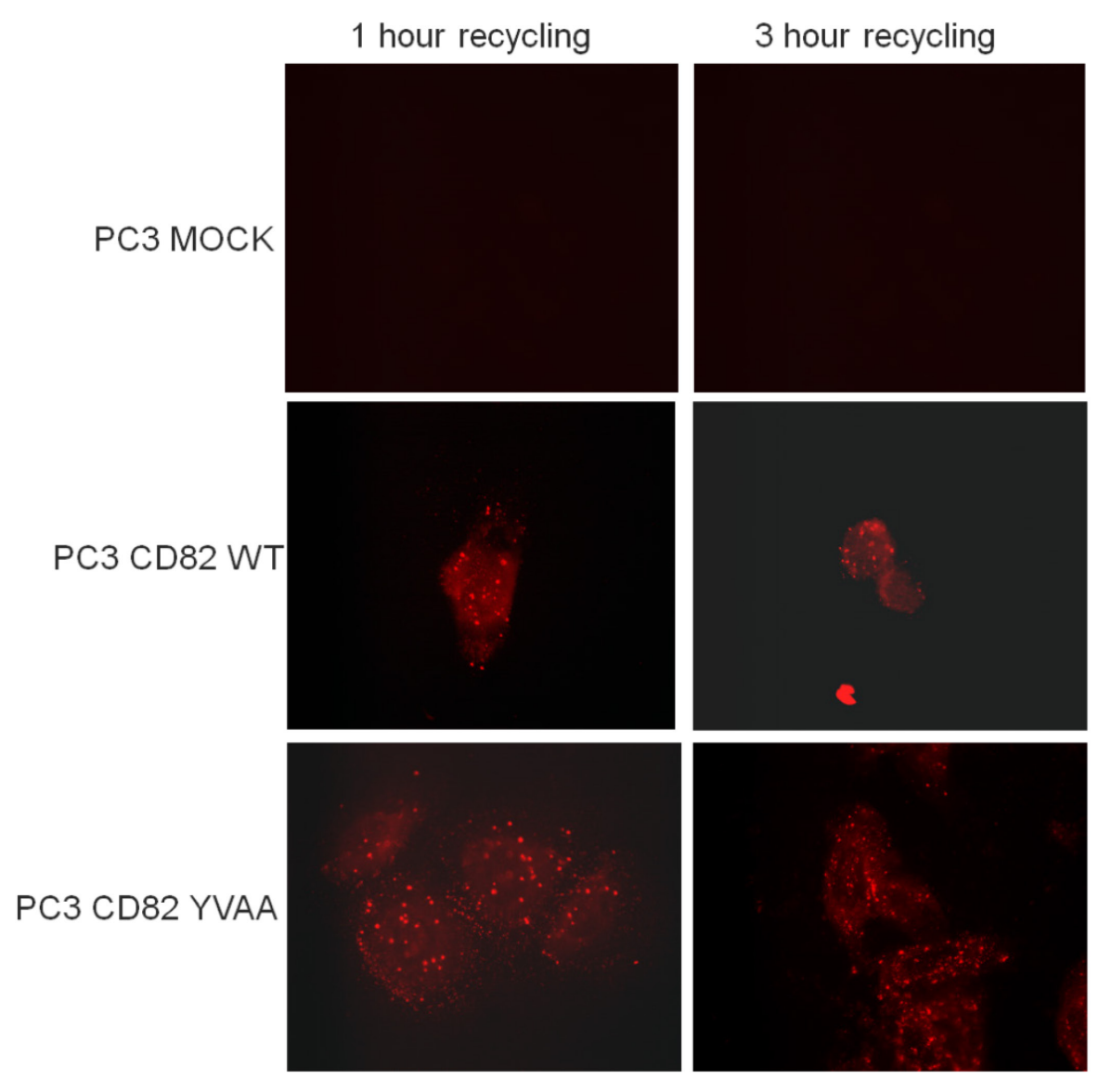

Figure 2-6. The YXXФ motif regulates CD82 recycling. PC3-Mock, -CD82 WT and -CD82 YVAA transfectant cells were plated onto glass coverslips and grown overnight in complete media. After the 60 -min incubation of CD82 $\mathrm{mAb}$ at $37^{\circ} \mathrm{C}$ for endocytosis and three acid-washes at RT for the removal of un-internalized $\mathrm{mAb}$, the transfectants were placed back at $37^{\circ} \mathrm{C}$ for 1 or $3 \mathrm{~h}$ for recycling. The cells were then further treated with acid-washes to remove the recycled mAbs. The cells were then fixed, permeabilized, and incubated with 2 nd $m A b$. Immunofluoresence images of the recycling assay were captured using a fluorescence microscope $(63 \mathrm{x})$. 
A

Reegcling Assy single Cell Quartifiection ofl inneges

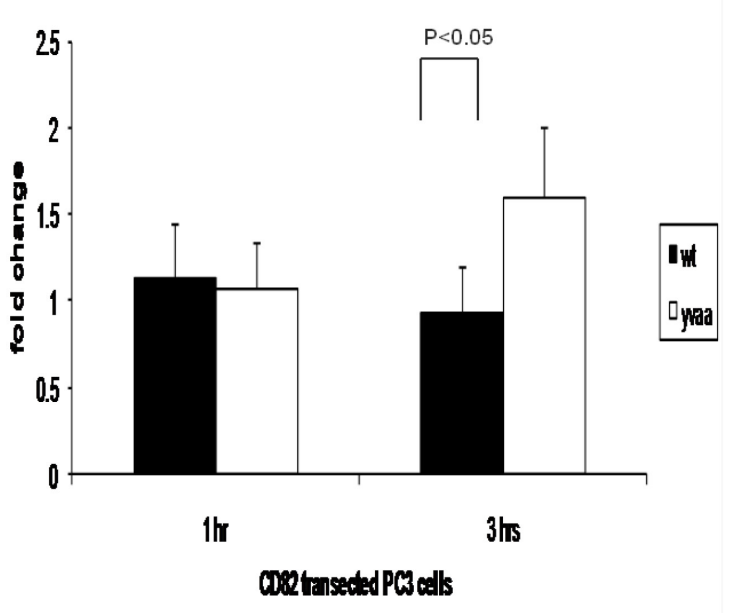

B

Regcling Assay using FACS analysis

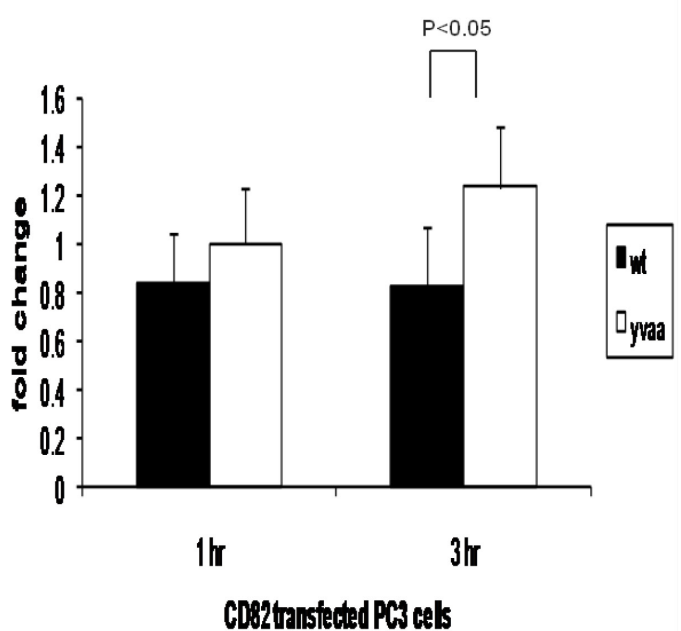

Figure 2-7. Mutation of the YXXФ motif regulates CD82 recycling. PC3-Mock, -CD82 WT and -CD82 YVAA transfectant cells were plated onto glass coverslips and grown overnight in complete media. After 60 -min incubation $37^{\circ} \mathrm{C}$ for endocytosis followed by acid-washing, the transfectants were placed back at $37^{\circ} \mathrm{C}$ for 1 or $3 \mathrm{~h}$ for recycling followed by acid-washing. The cells were then fixed, permeabilized, and incubated with $2 \mathrm{nd} \mathrm{mAb}$. A, immunofluoresence images of the recycling assays were acquired by a fluorescence microscope (63x), and CD82 endocytosis and recycling were quantified by counting the number of CD82-positive intracellular vesicles per cell (left panel). In each experiment, 50 CD82-positive cells were counted per transfectant. The histogram represents the number of CD82 positive vesicles remaining inside the cells after recycling divided by the number of vesicles per cell after internalizatoin. B, the recycling of CD82 in PC 3 transfectants was also analyzed in flow cytometry with the same principle and procedures described above (right panel). The FACS recycling data were normalized by dividing the mean flouresnece intensity after $1 \mathrm{hr}$ or $3 \mathrm{hr}$ by the mean flouresnece intensity after internalization. Each histogram represents the mean and standard deviation of recycling from four experiments. 
immunofluorescence-based recycling assay. For CD82 WT cells, CD82 proteins were recycled slowly and became gradually decreased intracellulary during 3 hour recycling period. For CD82 YVAA cells, the intracellularly remained CD82 mutant proteins even became increased after 3 hour recycling. In addition, in Du145-Mock, -CD82 WT, and -CD82 YVAA transfectants, we obtained the same results using the flow cytometry based recycling assay (data not shown).

The recycling data suggests that the function of the YXXФ motif of CD82 is to regulate its intracellular trafficking, specifically recycling. The increase of intracellular CD82 YVAA mutant proteins after 3-hour recycling suggests that some of the internalized CD82 YVAA mutant proteins may traffic to the acidic vesicular compartment and therefore cannot be detected by CD82 mAb, the probe for both endocytosis and recycling assays. The CD82 YVAA mutant proteins may gradually traffic out of the acidic vesicular compartment after the 3 hour incubation at $37^{\circ} \mathrm{C}$ for recycling and subsequently become detectable by CD $82 \mathrm{mAb}$. As a result, more CD82 staining was found intracellularly in CD82 YVAA transfectant after 3-hour recycling than after endocytosis or 1-hour recycling. Nevertheless, the mutation in the YХХФ motif of CD82 appears to redirect CD82 from the recycling back to the plasma membrane. The data presented in Figure 2-7 represents the average numbers or levels of CD82-positive intracellular vesicles per cell after 1 and 3 hour recycling. In Figure 2-7A, the quantitated data of recycling were obtained from the immunofluorescence images. Figure 2-7B represents the mean fluorescent intensity of the CD82 staining that remained intracellular after recycling. This data suggests that the function of the YXXФ motif of CD82 is to regulate its intracellular trafficking, specifically recycling.

\subsubsection{The mutation of the YXXФ motif does not disrupt the trafficking of CD82 to the late endosomal and lysosomal compartments}

The YXXФ motif is not only important for the regulation of internalization but also an important regulator of lysosomal trafficking. CD63 is a well established marker for the late endosomal and lysosomal compartments. We analyzed the co-localization of CD82 with the CD63-positive compartments, as shown in Figure 2-8.

Immunofluorescence images revealed that both CD82 WT and CD82 YVAA are able to localize to the CD63 positive compartments to similar extents, as shown in Figure 2-8A. The co-localization analysis revealed that 36 percent of both CD82 WT and YVAA were co-localized with CD63 in the late endosomal and lysosomal compartments, Figure 2-8B.

Since CD63 is a marker for the late endosome and lysosome, we further analyzed the trafficking of CD82 to each of the separate compartments. We analyzed the 

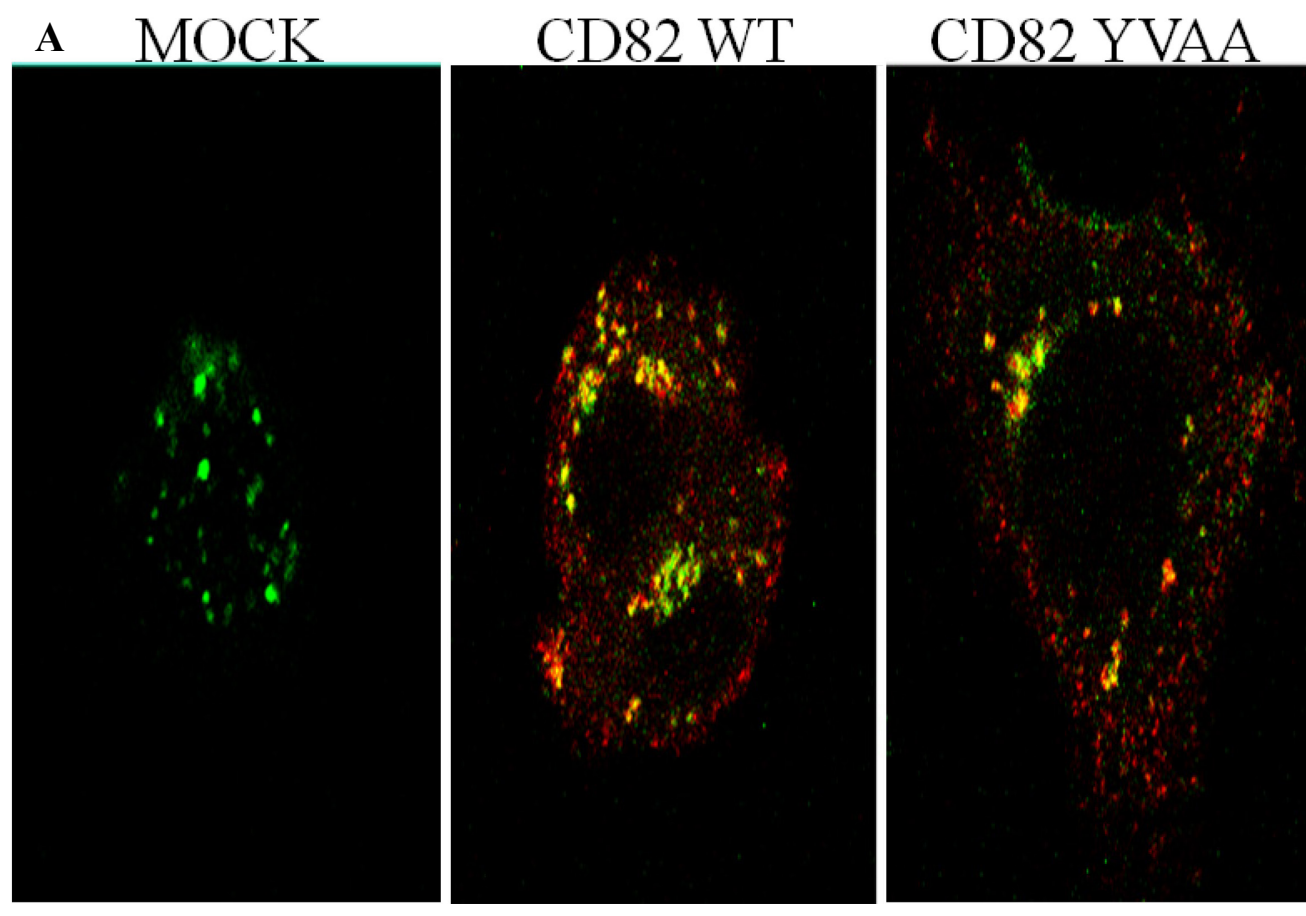

B

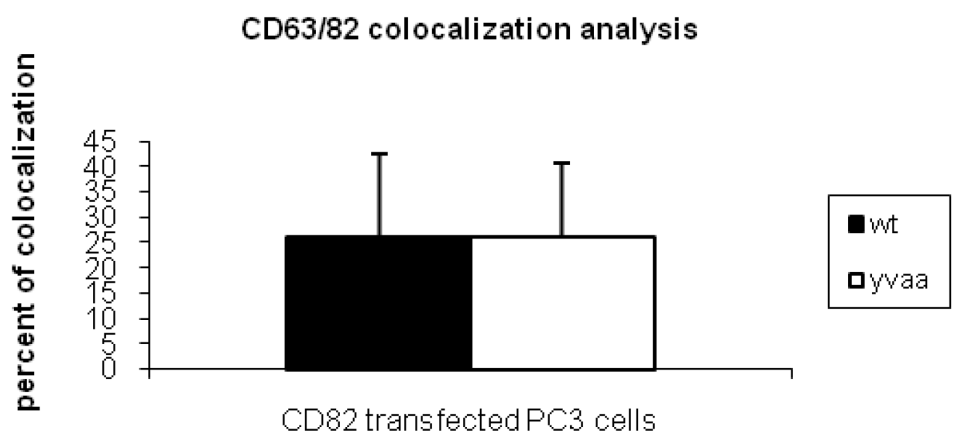

Figure 2-8. The mutation of the YXXФ motif does not disrupt CD82 trafficking to $C D 63$-positive late endosomal and lysosomal compartments. $A$, the

PC3 -MOCK -CD82 WT and -CD82 YVAA transfectant cells were plated onto glass coverslips and grown overnight in complete media. The cells were fixed, permeablized, and then incubated with Alexa 594-conjugated CD82 mAb TS82b and FITC-conjugated CD 63 mAb. Digital images were acquired under a confocal microscope. B, The quantifications of CD82 colocalization with CD63 were carried out using LSM image software. Histogram represents the mean and standard deviation of colocalization levels from three experiments. 
colocalization of CD82 with lysobiphosphatic acid (LBPA), a late endosome/multivesicular body marker, Figure 2-9. As shown in Figure 2-9A, immunofluorescence analysis indicating that CD82 WT and CD82 YVAA both co-localize with LBPA at similar levels. Co-localization analysis, using LSM software, reveals that 32 and 35 percent of CD82 WT and YVAA localizes to the late endosome/multivesicular body, respectively, Figure 2-9B.

We also analyzed the localization of CD82 to lysosomes using a lysosome probe, LysoTracker. As shown in Figure 2-10, we discovered that markedly more CD82 YVAA proteins were localized to the acidic, lysosomal, compartments compared to CD82 WT. Figure 2-10A reveals that more CD82 WT proteins appear to localize to the cell periphery whereas the CD82 YVAA localizes mainly to the intracellular compartments. Co-localization analysis, using LSM software, revealed that 12 and 43 percent of CD82 WT and YVAA co-localize with LysoTracker, respectively, Figure 2-10B.

These results indicate that the function of the YXXФ motif of CD82 is not to drive CD82 trafficking to the multivesicular bodies and lysosome to be degraded. Our studies showed that the function of the YXXФ motif of CD82 is to have CD82 recycled back to the plasma membrane or released into the extracellular space of the cells.

\subsubsection{CD82 proteins are likely recycled by Rab 11 recycling endosomes and the YVAA mutation reduced CD82 trafficking to Rab 11 recycling endosomes}

CD82 WT and YVAA mutant proteins have been found on the plasma membrane, in late endosomal compartments, and in lysosomal compartments. The trafficking of a transmembrane protein to late endosomes typically has two different fates: 1) being recycled back to the plasma membrane with the aid of Rab 4 or Rab11 or 2) being secreted to the extracellular milieu of the cells via exosomes.

Rab 4 and Rab 11 are the primary proteins responsible for recycling intracellular proteins back to the plasma membrane. Our early study revealed that significantly more CD82 proteins are localized in Rab 11 recycling endosomes than in Rab 4 recycling endosomes (Xu et al 2009), suggesting that CD82 is recycled through Rab 11 recycling endosome.

To investigate the recycling mechanism of CD82, we transiently expressed Rab 4 and Rab 11 wild type and dominant negative mutants in PC3 CD82 transfectants and discovered that CD82 uses the Rab 11 pathway for recycling back to the plasma membrane. Less than $10 \%$ of CD82 WT proteins colocalizes with Rab 4 at steady state 


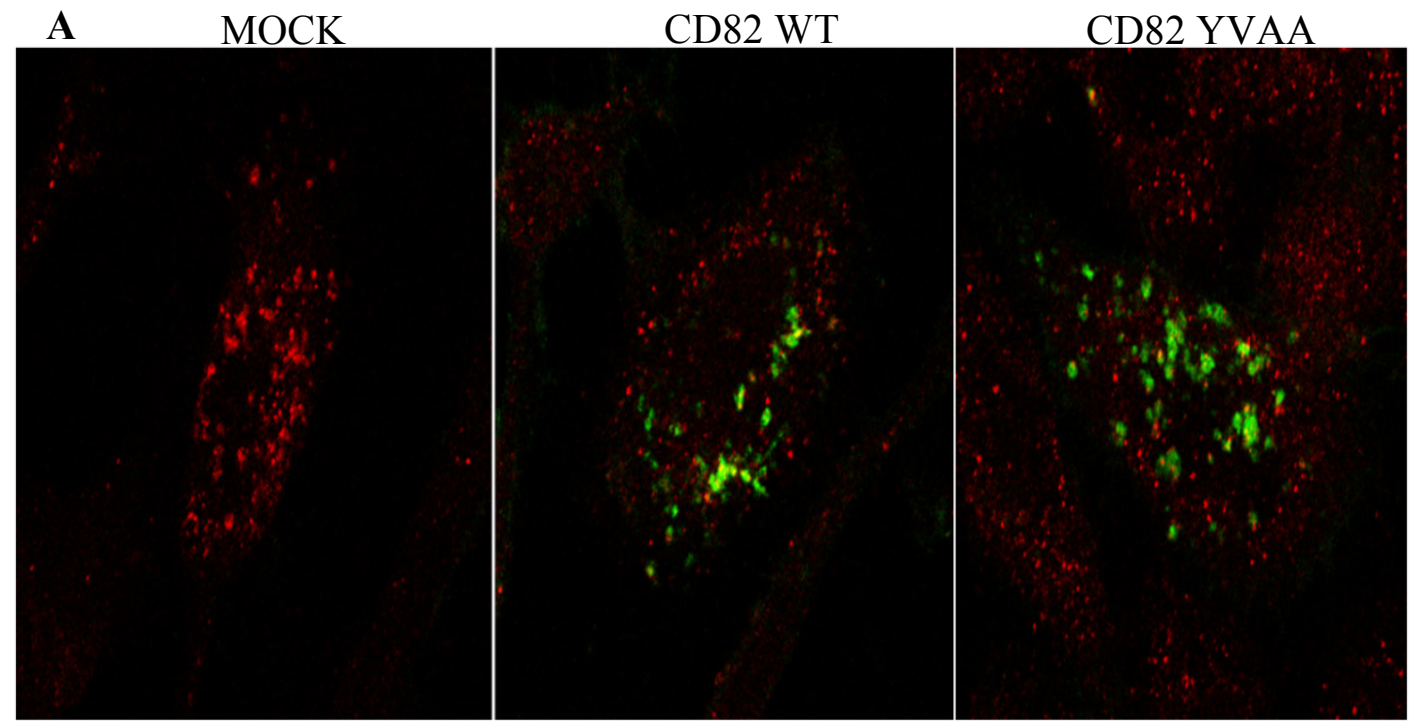

$\mathbf{B}$

\section{CD82 colocalization with LBPA}

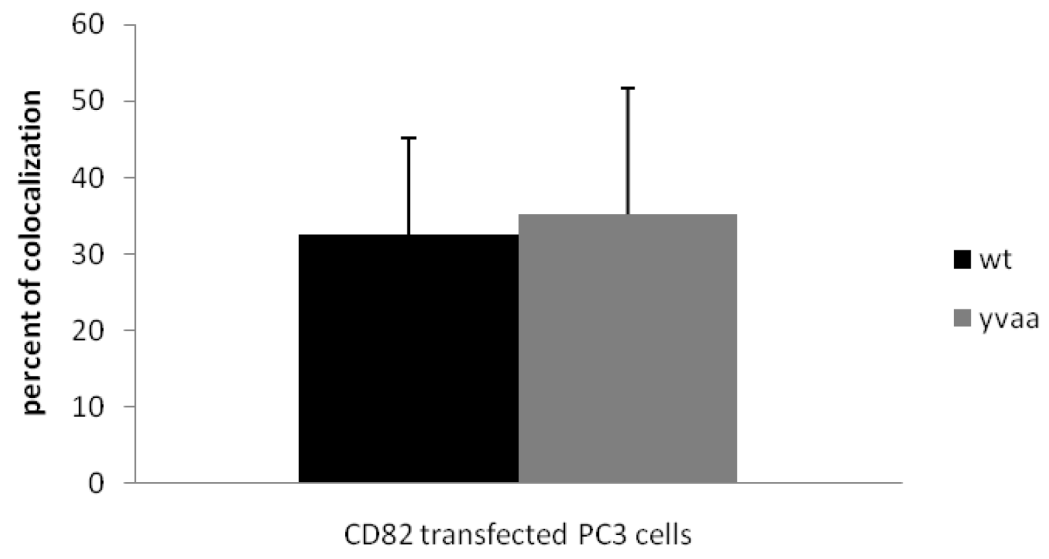

Figure 2-9. The mutation of the YXXФ motif does not disrupt CD82 trafficking to the multivesicular bodies (MVBs) of the cells. A, The PC3-MOCK -CD82 WT and -CD82 YVAA transfectant cells were plated onto glass coverslips and grown overnight in complete media. The cells were fixed, permeablized, and then incubated with Alexa 488-conjugated CD82 mAlb TS82 b and Alexa 594-conjugated LBPA $\mathbf{m A b}$. The images were captured using a confocal microscope. $\mathbf{B}$, the quantifications of CD82 colocalization with LBPA were carried out using LSM image software. Histogram represents the mean and standard deviation of colocalization levels from a total of 50 cells and three experiments. 

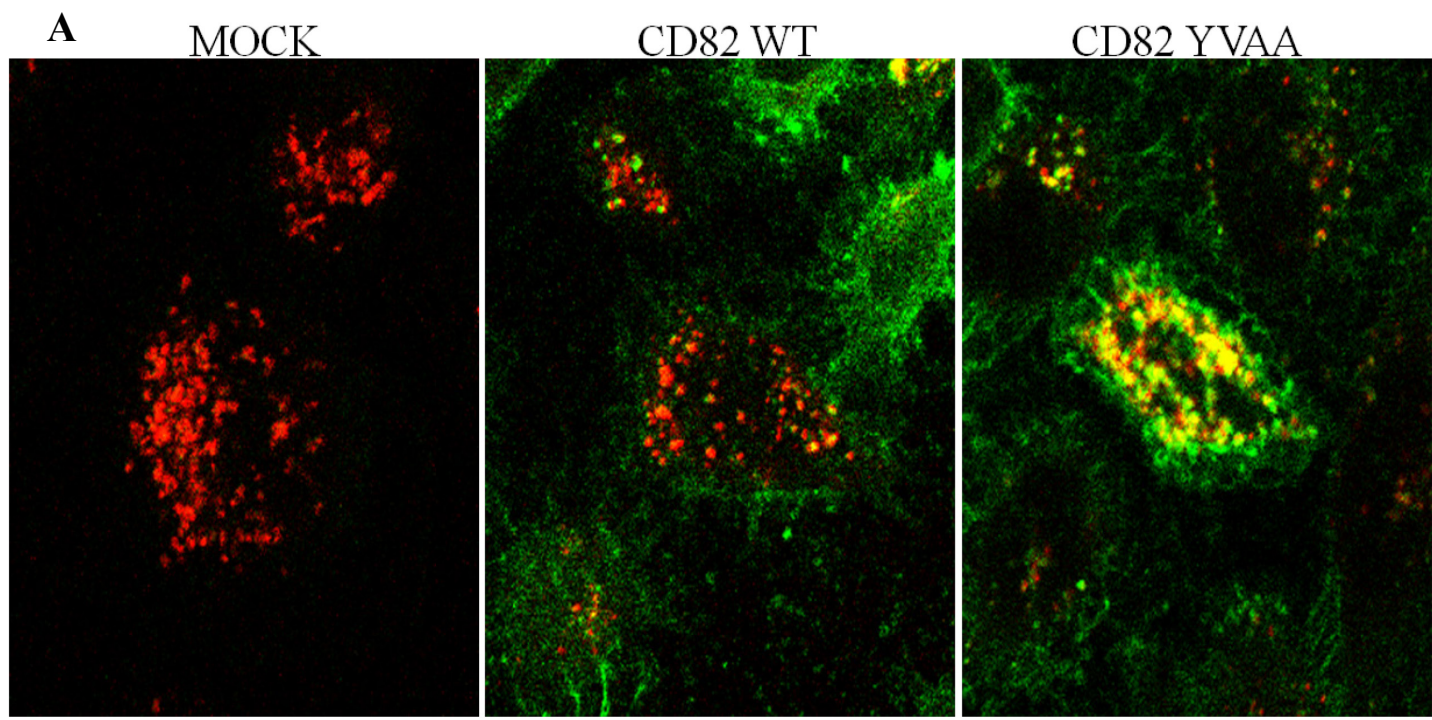

B

CD82/Lysotracker colocalization analysis

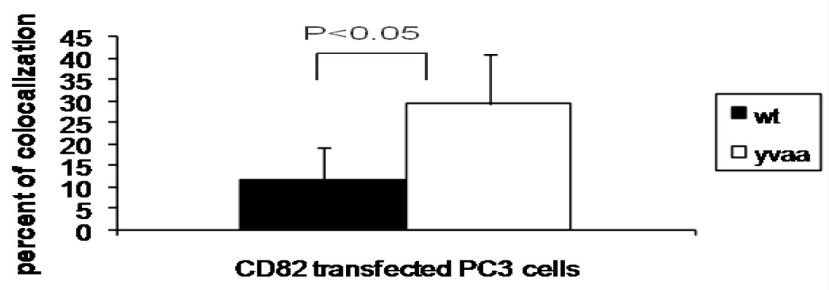

Figure 2-10. Mutation of the YXXФ motif directs CD82 trafficking to the lysosomal compartments of the cells. A, The colocalization of CD82 with LysoTracker. The PC3-MOCK -CD82 WT and -CD82 YVAA transfectant cells were plated onto glass coverslips, grown overnight in complete media, and incubated with Texas Red-conjugated LysoTracker at $37^{\circ} \mathrm{C}$ for $20 \mathrm{~min}$. The cells were fixed, permeabilized, probed with Alexa 488-conjugated CD82 mAb TS82b. The images were captured using a confocal microscope. Red, LysoTracker; Green, CD82. B, The quantifications of CD82 colocalization with LysoTracker were carried out using LSM image software. Histogram represents the mean and standard deviation of colocalization levels from a total of 50 cells and three experiments. 
whereas approximately $30 \%$ of CD82 WT colocalizes with Rab 11 (data not shown).

Furthermore, we observed that, after 90 minutes of internalization, a majority of CD82 WT is recycled; however quite a few CD82-positive vesicles still remained within the CD82 YVAA cells, as shown Figure 2-11. When the CD82 endocytosis was analyzed in the PC3 transfectant cells that express wild type or a dominant negative-mutant of Rab 11, the CD82 WT-positive vesicles accumulated when the dominant negative-mutant of Rab 11 was expressed, and CD82 WT proteins were colocalized readily with the Rab 11 dominant-negative mutant, Figure 2-11. There is not an alteration in accumulation of CD82 YVAA proteins in the cells that express the Rab 11 dominant-negative mutant, compared to the cells that express Rab 11 wild type, Figure 2-11A. The co-localization of CD82 proteins with either Rab 11 WT or Rab11 dominant-negative mutant becomes markedly diminished upon the YVAA mutation as shown in Figure 2-11B. This data strongly suggest that Rab 11 is responsible for CD82 recycling once CD82 reaches the late endosomes, and the YXXФ motif facilitates the trafficking of CD82 to Rab 11-positive recycling endosomes.

\subsubsection{CD82 proteins are released from PC3 cells via exosomes}

To investigate whether CD82 can be released via the exosomal compartment and determine the effect of YVAA mutation on exosomal release, we performed differential centrifugation on conditioned media from PC3 transfectant cells and found that CD82 WT proteins are highly enriched in the purified exosomes, compared to CD82 YVAA mutant proteins, as shown Figure 2-12. Flotillin, an exosomal marker, was used as a loading control and also displayed the exosomal enrichment In Figure 2-12A, the levels of flotillin reflect that the same amount of protein from the total cell lysate, conditioned media, and isolated exosomes was loaded from each transfectant.

In Figure 2-12B, we observed that transferrin receptor was not released via exosomes as predicted and therefore serves as the negative control. Figure 2-12B also reveals that tetraspanin CD81 is enriched in and released via exosomes and serves as a positive control. Similar levels of CD82 expression between PC3-CD82 WT and -CD82 YVAA transfectants can be found from total cell lysates, as shown in Figure 2-12C. Most importantly, as shown in Figure 2-12D, this experiment revealed that, in the purified exosomes, i.e., fraction 7, CD82 WT proteins were highly enriched and much more abundant compared to CD82 YVAA mutant proteins in the same fraction.

Taken together, this set of data suggest that the function of the YXXФ motif of CD82 is to direct 1) the trafficking of CD82 from the plasma membrane to the late endosome/multi-vesicular body, 2) the recycling of CD82 from late endosomes back to 

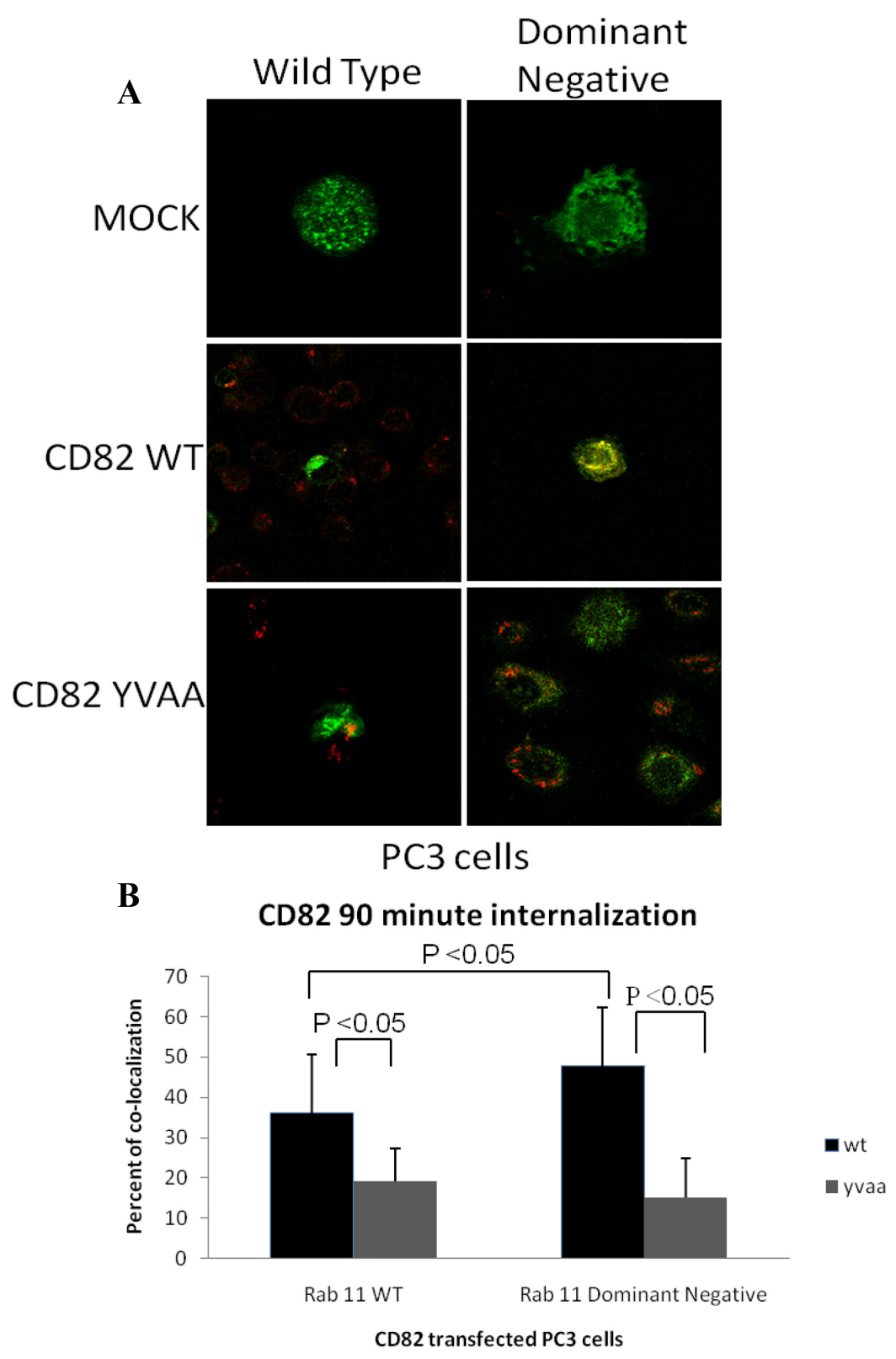

Figure 2-11. Mutation of the YXXФ motif disrupts CD82 trafficking to the Rab 11 positive compartments. A, For the co-localization of CD82 with Rab 11 WT or dominant negative mutant, the PC3 transfectant cells were transiently transfected with Rab 11 WT-GFP or Rab 11 domimant negative mutant-GFP. After 48 hours, the cells were fixed, permeablized, blocked, incubated with Alexa 594-conjugated TS82b for 1.5 hours, washed, and then mounted. The images were captured using a confocal microscope. B, Quantifications of the colocalization of CD82 with Rab 11 WT or dominant negative mutant was carried out using LSM image software to assess the overlapped fluorescent intensities of CD82 and Rab11 staining. The histogram represents the average and standard deviation of three experiments. 
A

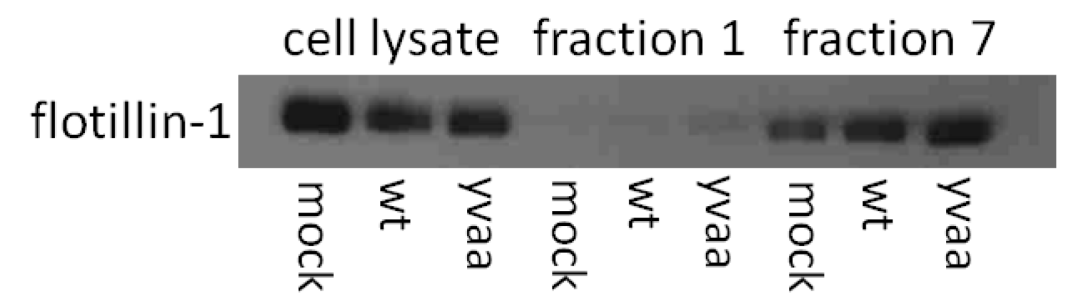

B
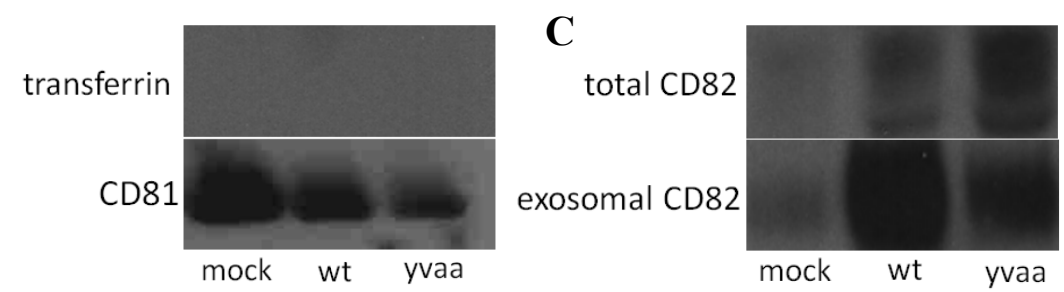

D

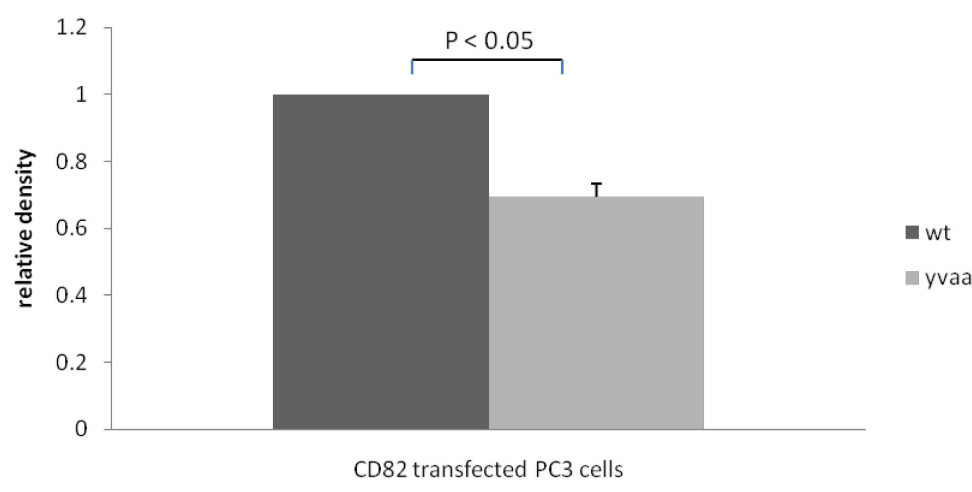

Figure 2-12. The effects of CD82 overexpression and CD82 YVAA mutation on exosomal content. The PC3-Mock, -CD82 WT, and -CD82 YVAA mutant transfectant cells were grown in incomplete DMEM for 48-72 hours. The culture supernatants were harvested and subjected to differential centrifugations for exosome purification (see the details in "Materials and Methods"). The exosome preparation and total cell lysate samples were dissolved in Laemmli sample buffer, separated by SDS-PAGE, and analyzed in Western blot. A, to ensure that we obtained purified exosomes, we probed the centrifugation fractions for the exosomal marker flotillin. Fraction 1 represents the total amount contained within the media released from the cells, fraction 7 represents the isolated exosome fraction from the 100,00x $g$ centrifugation step. B, we also analyzed the 100,00x $g$ fraction for tetraspanin CD81, a protein enriched in exosomes, and transferrin a proteins absent from exosomes, as shown in top panel and bottom panel, respectively. $\mathbf{C}$, we also analyzed total cellular CD82 expression from whole cell lysate (top panel) and the CD82 found in the 100,00x $g$ fraction (bottom panel). D, The band intensity for the CD82 proteins released with exosomes, as shown in the bottom panel of $\mathrm{C}$, was quantified using Image J analysis. 
the plasma membrane, and 3) the releases of CD82 via exosomes.

\subsubsection{The mutation of the YXXФ motif of CD82 does not affect the cell surface expression levels of $\mathrm{CD82}$-associated tetraspanins but up-regulates the cell surface expression levels of integrins $\alpha 2 \beta 1, \alpha 3 \beta 1$, and $\alpha 5 \beta 1$}

CD82 and other tetraspanins sequesters other cell surface proteins such as integrins to form TEMs at the plasma membrane (Liu and Zhang 2006; Richardson 2011). It is suggested that the motility-suppressive function of CD 82 is mediated by its associated transmembrane proteins that are located within the CD82-positive TEMs (Berditchevski, 2001; Berditchevski and Odintsova 2007; Helmer 2003; Liu and Zhang 2006; Richardson 2011). The alteration in CD82 trafficking due to the YVAA mutation may also affect the steady-state cellular distribution and trafficking of CD82-associated TEM components.

Therefore we analyzed the cell surface expression of CD82-associated tetraspanins and integrins by flow cytometry, and the results are shown in Figure 2-13. The YVAA mutation led to the significant up-regulation of integrins $\alpha 2 \beta 1, \alpha 3 \beta 1$, and $\alpha 5 \beta 1$ at the surface of PC3 cells, compared to the CD82 WT transfectant cells,

Figure 2-13A. The cell surface level of integrin $\alpha 6 \beta 1$ did not exhibit a significant change in $\mathrm{PC} 3$ transfectants.

However, the mutation of the YXXФ motif of CD82 did not significantly alter the cell surface expression of CD82-assocaited tetraspanins such as CD9, CD63, CD81, and CD151, Figure 2-13B, indicating that CD82 YХXФ motif or CD82 trafficking determined by this motif is not required for the cell surface expression of these tetraspanins in $\mathrm{PC} 3$ cells.

The mean fluorescent intensity of these proteins in the PC3-CD82WT and PC3-CD82YVAA transfectants, presented in Figure 2-13, were normalized by the intensity of the corresponding proteins in the MOCK cells.

\subsubsection{The mutation of CD82 YXXФ motif alters the trafficking of integrins $\alpha 3 \beta 1$, $\alpha 6 \beta 1$, and $\alpha 5 \beta 1$ into CD82-positive compartments}

We then analyzed the role of the CD82 YXXФ motif in the trafficking of these integrins. By examining the internalization of integrins into the intracellular vesicular compartments, we determined the effect of CD82 YVAA mutation on integrin endocytosis. By examining the colocalization of internalized integrins with CD82 in the intracellular vesicular compartments, I determined how much of the integrin traffics into 
A

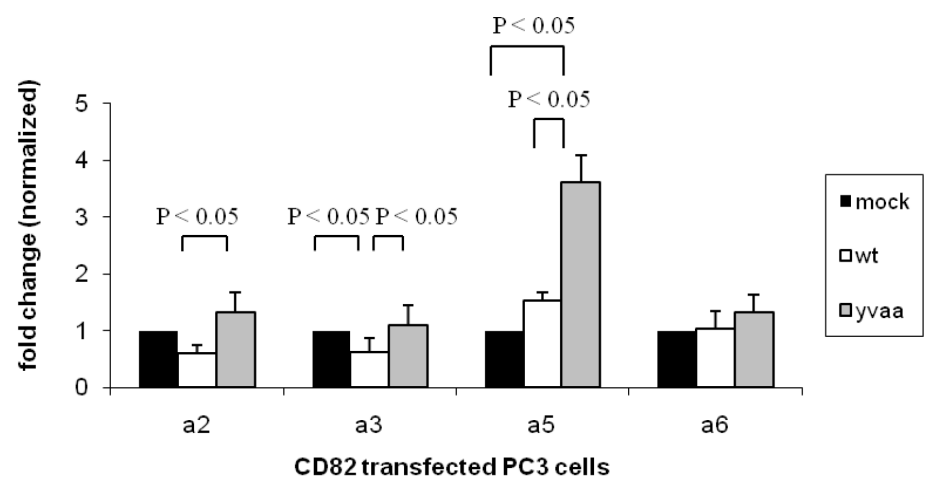

B

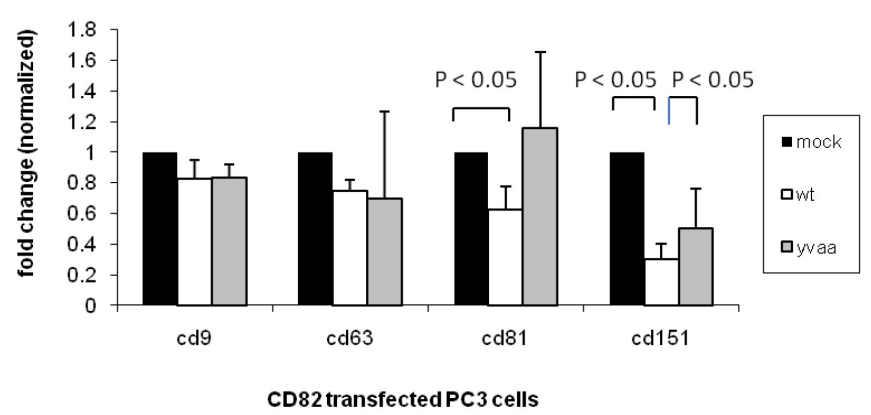

Figure 2-13. Mutation of the YXXФ motif of CD82 does not affect the cell surface expression of CD82-associated tetraspanins but up-regulates the cell surface levels of integrins $\alpha 2, \alpha 3$, and $\alpha 5$ in PC3 cells. For flow cytometry, confluent cells were detached, washed with 1xPBS, and blocked with $2 \%$ goat serum on ice for 1 hour to block the non-specific binding. A, cells were incubated with $1 \mu \mathrm{g} / \mathrm{ml}$ of primary $\mathrm{mAb}$ such as integrin $\alpha 2 \mathrm{mAb}$ IIE10, integrin $\alpha 3 \mathrm{mAb}$ A3X8, $\alpha 5 \mathrm{mAb}$ BIIG2, integrin $\alpha 6$ A6BB on ice for 1 hour followed by PBS washes. The cells were then labeled with appropriate flurochrome-conjugated secondary $\mathrm{Ab}$ on ice for 30 mins followed by extensive PBS washes. Cell surface expression of the proteins was analyzed using a FACS Calibur flow cyotmeter (BD Biosciences). B, cells were incubated with $1 \mu \mathrm{g} / \mathrm{ml}$ of primary mAb such as tetraspanins mAbs CD63, CD81, CD151, and CD9 on ice for 1 hour followed by PBS washes. The cells were then labeled with appropriate flurochrome-conjugated secondary $\mathrm{Ab}$ on ice for 30 mins followed by extensive PBS washes. Cell surface expression of the proteins was analyzed using a FACS Calibur flow cyotmeter (BD Biosciences). The histograms represent the mean and standard deviation of at least three independent experiments. 
CD82-positive compartments after endocytosis.

Upon the mutation of the YXXФ motif of CD82, the number of integrin $\alpha 2 \beta 1$-positive vesicles per cell as well as the colocalization of integrin $\alpha 2 \beta 1$ with CD82 was not altered in PC3 transfectant cells, as shown in Figure 2-14A. The average number of integrin $\alpha 2 \beta 1$-positive vesicles per cell, following integrin $\alpha 2 \beta 1$ internalization, was 18, 16, and 21 for the MOCK, CD82 WT, and CD82 YVAA transfectant cells, respectively, Figure 2-14B, left panel, suggesting that the YVAA mutation has no impact on integrin $\alpha 2 \beta 1$ endocytosis. Furthermore, the colocalization of internalized integrin $\alpha 2 \beta 1$ with CD82 was unaffected by the mutation of CD82 YXXФ motif, Figure 2-14B, right panel, suggesting that the YVAA mutation has no impact on integrin $\alpha 2 \beta 1$ trafficking into CD82-positive compartment.

The mutation of the YXXФ motif led to an increase in the number of integrin $\alpha 3 \beta 1$-positive vesicles per cell, as shown in Figure 2-15A. Visual counting revealed that the PC3-MOCK and PC3-CD82 YVAA transfectants contained significantly more integrin $\alpha 3 \beta 1$-positive vesicles compared to the PC3-CD82 WT transfectants, as shown in the left panel of Figure 2-15B. There was not a significant difference in the number of the vesicles per cell between the PC3-MOCK and PC3-CD82 YVAA transfectants. These data suggest that CD82 inhibits the endocytosis of integrin $\alpha 3 \beta 1$ while the YVAA mutation disrupted this inhibition. As presented in the right panel of Figure 2-15B, we also analyzed the CD82-colocalized, internalized integrin $\alpha 3 \beta$. The quantification using LSM software revealed that the integrin $\alpha 3 \beta 1$-positive vesicles within the PC3-CD82 WT and -CD82 YVAA transfectants were partially colocalized with the CD82-positive compartments. Compared to the CD82 WT cells, we found that significantly more internalized integrin $\alpha 3 \beta 1$ proteins were colocalized with CD82 proteins in the CD82 YVAA cells.

In contrast to integrin $\alpha 2 \beta 1$, the mutation of CD82 YXX $\Phi$ motif led to an accumulation of integrin $\alpha 5 \beta 1$-positive vesicles inside PC3 cells, compared to the MOCK and CD82WT cells, as shown in Figure 2-16A. On the average, only 8 integrin $\alpha 5 \beta 1$-vesicles per cell were found in the MOCK transfectants, 7 were found in the CD82WT transfectants, whereas 23 were found in the CD82 YVAA transfectants, Figure 2-16B, left panel. Not surprisingly, significantly more integrin $\alpha 5 \beta 1$ proteins were localized into the CD82-positive compartments, Figure 2-16B. Approximately 17\% of the internalized integrin $\alpha 5 \beta 1$ proteins were colocalized with CD82 proteins in PC3CD82 WT cells whereas 50\% colocalized with CD82 in PC3-CD82 YVAA cells, suggesting that either increased endocytosis or decreased recycling of integrin $\alpha 5 \beta 1$ resulted from CD82 YVAA mutation. 

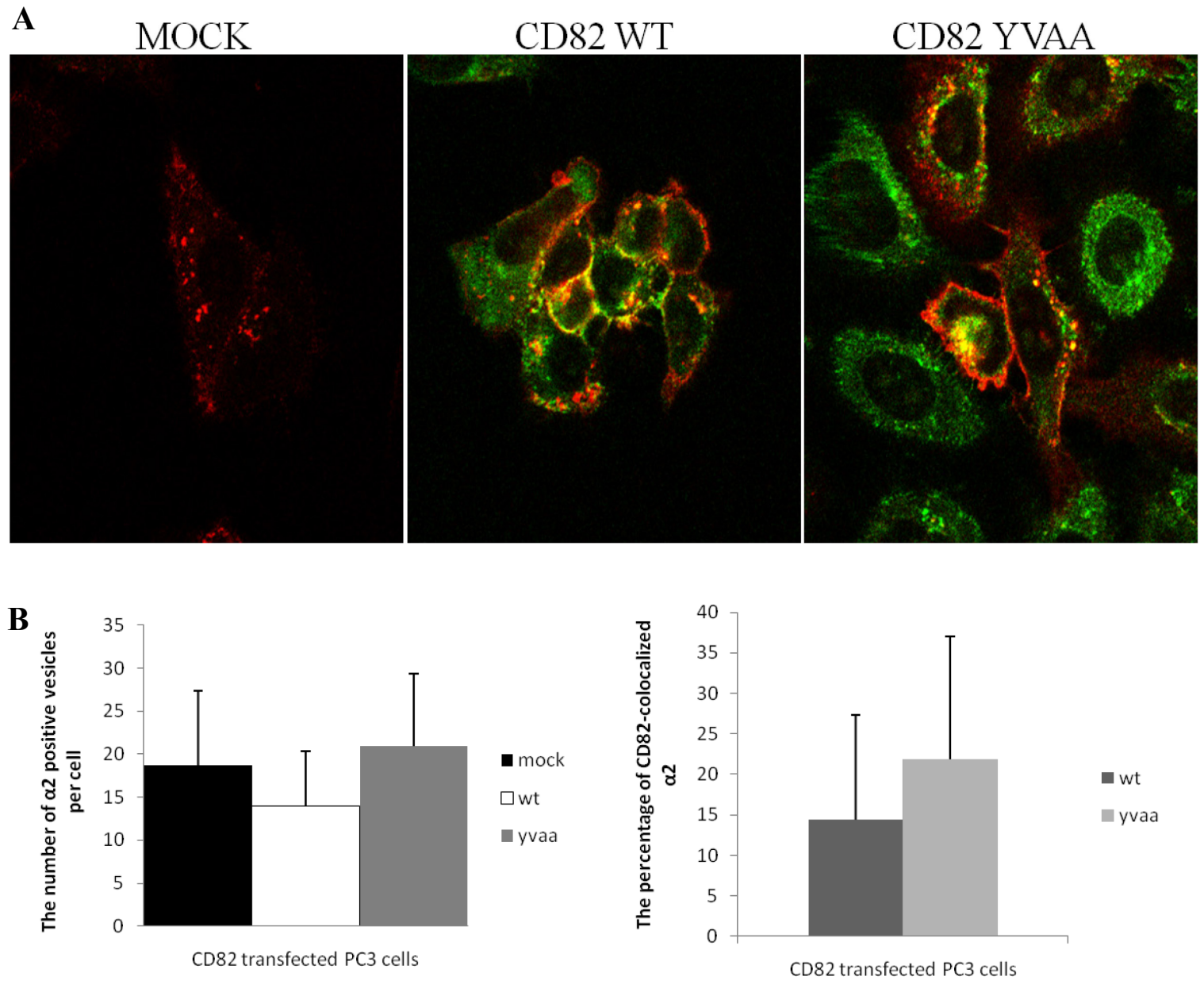

Figure 2-14. Mutation of the YXXФ motif of CD 82 does not affect the number of vesicles per cell or the colocalization of $\alpha 2$ with $C D 82$ after internalization. $A$, the PC3-Mock, -CD82 WT, and -CD82 YVAA transfectant cells were plated onto glass coverslips and grown overnight in complete media. The cells were incubated with integrins $\alpha 2$ at $4^{\circ} \mathrm{C}$ for $1 \mathrm{~h}$. The cells were then allowed to internalize the bound $\mathrm{mAb}$ by the incubation at $37^{\circ} \mathrm{C}$ for $1 \mathrm{~h}$, followed by acid washes to remove un-internalized mAbs. The cells were fixed, permeablized, and incubated with Alexa 594-conjugated 2nd mAb. The cells were then labeled with an Alexa 488 conjugated CD $82 \mathrm{mAb}$ and analyzed using a confocal microscope. $\mathbf{B}$, the number of $\alpha 2$ positive vesicles were visually counted, left panel, and the quantifications of $\alpha 2$ colocalization with CD82 were carried out using LSM image software (right panel). The histograms represent the mean and standard deviation from a total of 50 cells in three independent experiments. 
A

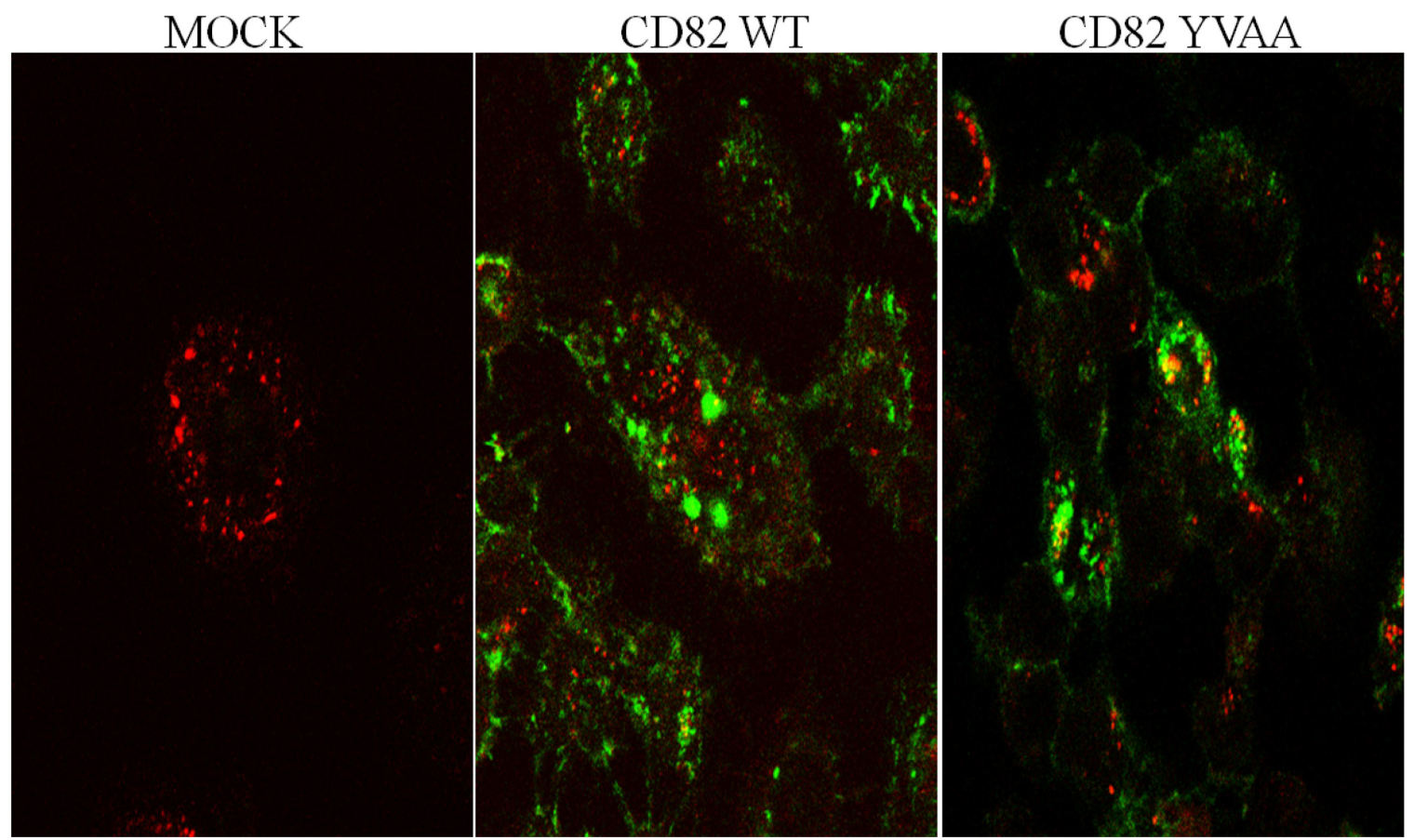

B
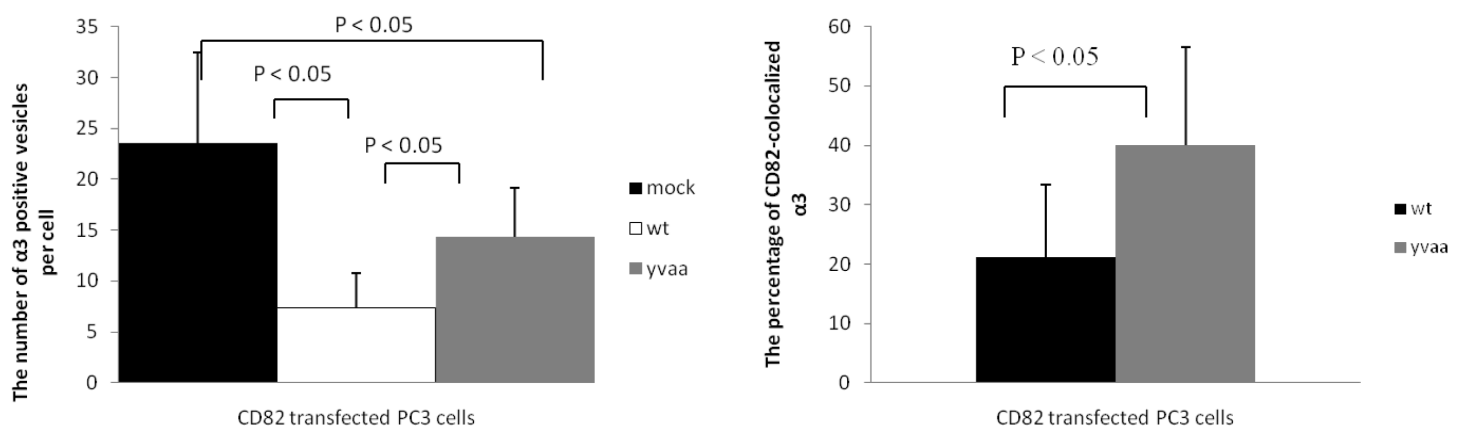

Figure 2-15. Mutation of the YXXФ motif of $C D 82$ leads to an accumulation of $\boldsymbol{\alpha} 3$ vesicles after internalization. A, the PC3-Mock, -CD82 WT, and -CD82 YVAA transfectant cells were plated onto glass coverslips and grown overnight in complete media. The cells were incubated with integrins $\alpha 3$ at $4^{\circ} \mathrm{C}$ for $1 \mathrm{~h}$. The cells were then allowed to internalize the bound $\mathrm{mAb}$ by the incubation at $37^{\circ} \mathrm{C}$ for $1 \mathrm{~h}$, followed by acid washes to remove un-internalized mAbs. The cells were fixed, permeablized, and incubated with Alexa 594-conjugated 2nd mAb. The cells were then labeled with an Alexa 488 conjugated CD82 mAb and analyzed using a confocal microscope. B, The number of $\alpha 3$ positive vesicles were visually counted, left panel, and the quantifications of $\alpha 3$ colocalization with CD82 were carried out using LSM image software (right panel). The histograms represent the mean and standard deviation from a total of 50 cells in three independent experiments. 

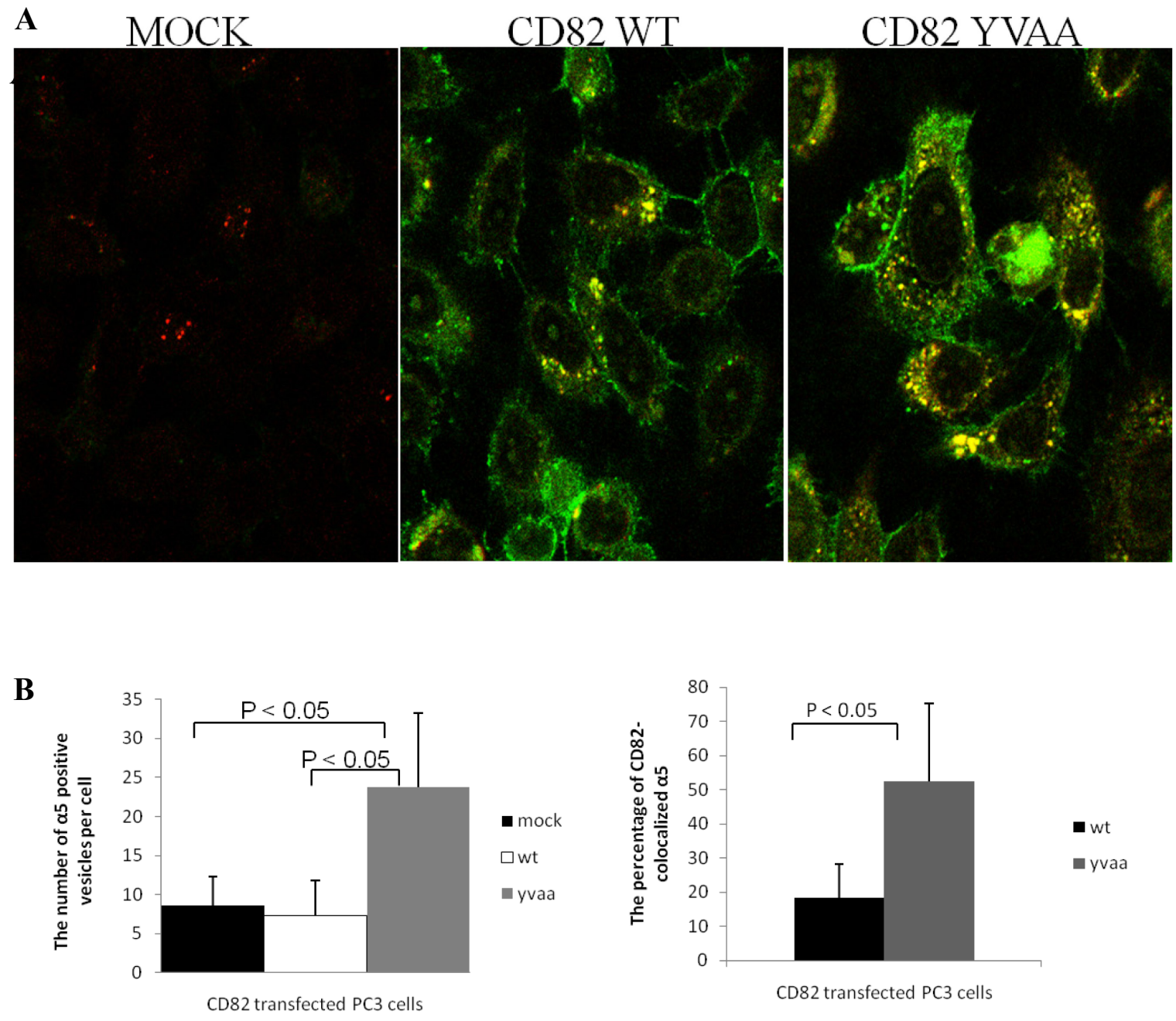

Figure 2-16. Mutation of the YXXФ motif of CD82 leads to an accumulation of 0,5 vesicles after internalization. A, the PC3-MOCK, -CD82 WT, and -CD82 YVAA transfectant cells were plated onto glass coverslips and grown overnight in complete media. The cells were incubated with integrin $\alpha 5$ at $4^{\circ} \mathrm{C}$ for $1 \mathrm{~h}$. The cells were then allowed to internalize the bound $\mathrm{mAb}$ by the incubation at $37^{\circ} \mathrm{C}$ for $1 \mathrm{~h}$, followed by acid washes to remove un-internalized mAbs. The cells were fixed, permeablized, and incubated with Alexa 594-conjugated 2nd mAb. The cells were then labeled with an Alexa 488 conjugated CD82 mAb and analyzed using a confocal microscope. B, The number of $\alpha 5$ positive vesicles were visually counted, left panel, and the quantifications of $\alpha 5$ colocalization with CD82 were carried out using LSM image software (right panel). The histograms represent the mean and standard deviation from a total of 50 cells in three independent experiments. 
The mutation of CD82 YXXФ motif did not markedly alter the number of integrin $\alpha 6$-positive vesicles per cell after the $1 \mathrm{~h}$ endocytosis of integrin $\alpha 6$ in PC3 cells, as shown in Figure 2-17A. Roughly, 18, 15, and 14 integrin $\alpha 6$-vesicles per cell can be found in the MOCK, CD82WT, and CD82YVAA transfectants, respectively, Figure 2-17B, left panel. However, the mutation of CD82 YXXФ motif led to the increased internalization of integrin $\alpha 6$ into CD82-positive vesicles, as shown in the right panel of

Figure 2-17B. Approximately $33 \%$ of the internalized integrin $\alpha 6$ proteins were localized into CD82-positive compartments in the CD82 YVAA transfectant cells compared to $22 \%$ in the CD82 WT transfectant cells.

\subsection{SUMMARY}

In order to investigate 1) the role of the YXXФ motif in CD82 trafficking and 2) the role of the intracellular trafficking in the motility-suppressive function of CD82, we mutated the tyrosine-based trafficking motif of CD82 and generated CD82 YVAA mutant. We generated the stable transfectants of MOCK, CD82 WT, and CD82 YVAA mutant in PC3 and Du145 cells. Similar levels of CD82 expression were reached in the CD82 WT and CD82 YVAA transfectants. We analyzed the steady state localization, internalization, and recycling of CD82 to determine the role of the YXXФ motif in CD82 trafficking. We found that the YХXФ motif of CD82 does not play a role in the internalization but is responsible for directing the intracellular trafficking, summarized in Table 2-1, and the recycling of CD82 back to the plasma membrane. We also found that CD82 can be released to the extracellular environment by exosomes and the YXXФ motif of CD82 regulates the exosomal release of CD82. After being released to the extracellular space, CD82 has the potential to elicit its function on neighboring cells. 

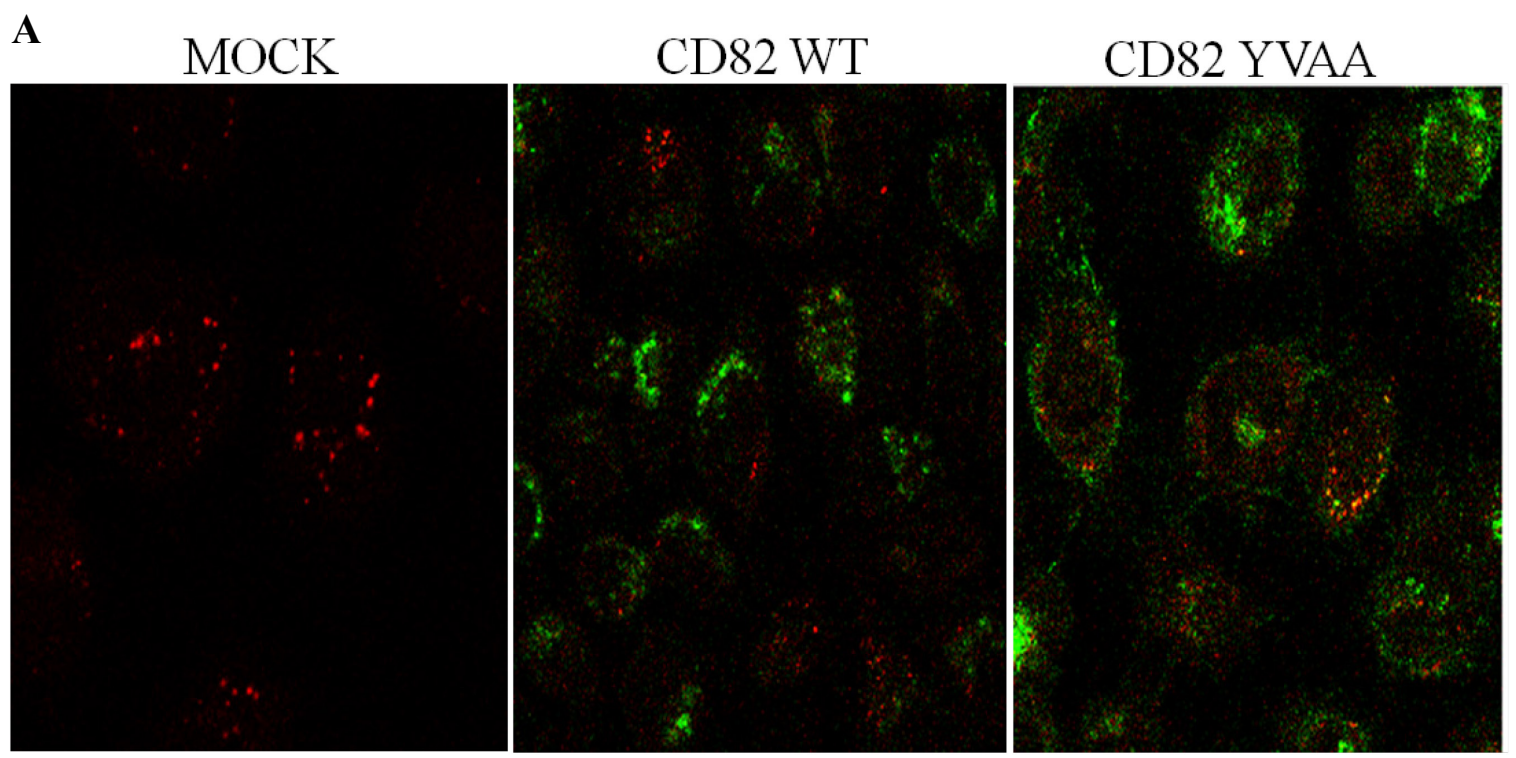

B
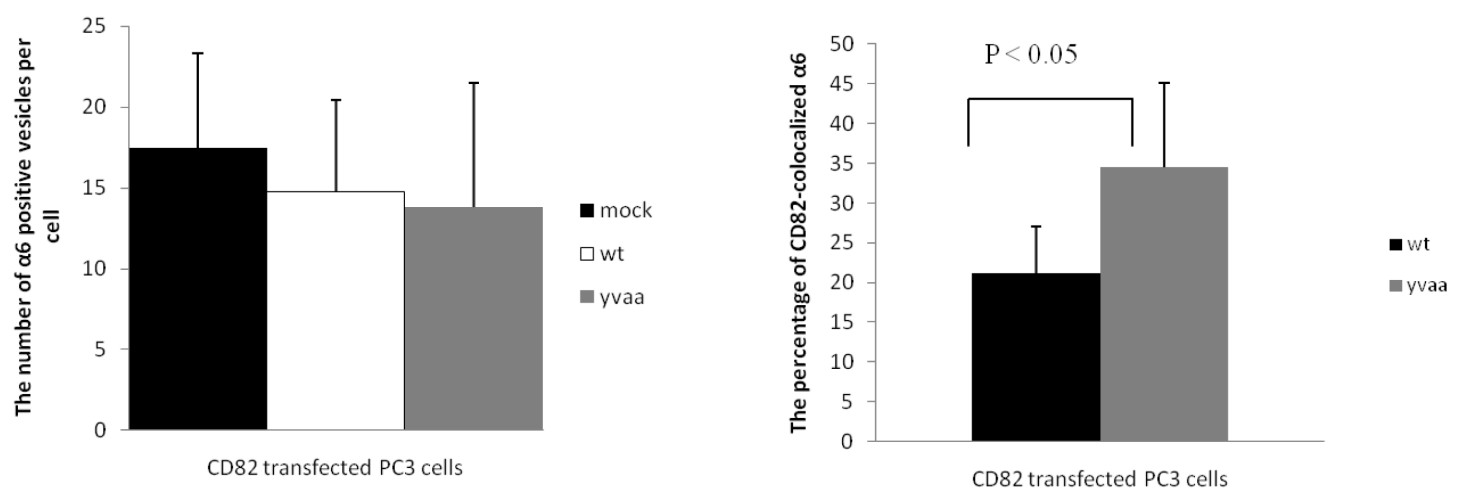

Figure 2-17. Mutation of the YXXФ motif of CD82 leads to an increase in CD82/a6 colocalization after internalization. A, the PC3-MOCK, -CD82 WT, and-CD82 YVAA transfectant cells were plated onto glass coverslips and grown overnight in complete media. The cells were incubated with integrins $\alpha 6$ at $4^{\circ} \mathrm{C}$ for $1 \mathrm{~h}$. The cells were then allowed to internalize the bound $\mathrm{mAb}$ by the incubation at $37^{\circ} \mathrm{C}$ for $1 \mathrm{~h}$, followed by acid washes to remove un-internalized mAbs. The cells were fixed, permeablized, and incubated with Alexa 594-conjugated 2nd mAb. The cells were then labeled with an Alexa 488 conjugated CD $82 \mathrm{mAb}$ and analyzed using a confocal microscope. B, The number of $\alpha 6$ positive vesicles were visually counted, left panel, and the quantifications of $\alpha 6$ colocalization with CD82 were carried out using LSM image software (right panel). The histograms represent the mean and standard deviation from a total of 50 cells in three independent experiments. 
Table 2-1. The percentage of CD82 colocalization with intracellular organelle markers.

\begin{tabular}{ccc}
\hline Percent of colocalization with: & CD82 WT & CD82 YVAA \\
\hline Early Endosomes & & \\
EEA1 & 41 & 24.5 \\
Late Endosomes & 26 & 26 \\
CD63 & 32 & 35 \\
LBPA & & \\
Lysosomes & 12 & 35 \\
Lysotracker & \\
\hline
\end{tabular}

Table 2-1 summarizes the quantitative data from the colocalization studies found in Chapter 2. 


\section{CHAPTER 3. THE YXXФ MOTIF-MEDIATED REGULATION OF THE INTRACELLULAR TRAFFICKING OF CD82}

\subsection{INTRODUCTION}

Cell migration is a highly integrated, multi-step, locomotive cellular process, which orchestrates cellular morphogenesis, contributes to tissue repair, and drives disease progression. Understanding the role of cell migration in cancer progression is of the utmost importance. De-regulation of cell migration can result in cancer metastasis, which is responsible for $90 \%$ of cancer-related deaths. Cancer cell migration is typically regulated by growth factors, chemokines, matrix-degrading enzymes, and cell-cell and cell-matrix adhesion molecules. Earlier studies from our lab and elsewhere revealed that CD82 inhibits cell movement by down-regulating the signaling initiated from growth factor receptors and integrins and attenuating integrin-dependent cell-matrix adhesion (Richardson 2011).

In epithelial cells, cell-cell adhesion is most commonly regulated by cadherin, a family of calcium dependent cell-cell adhesion proteins. Cell-cell adhesion can also be mediated via calcium-independent cell-cell adhesion molecules such as members of Ig Superfamily (IgSF) proteins. Whether cell-cell adhesion contributes to the ability of CD82 to inhibit cancer metastasis is not clear. Mechanistically, it is possible that CD82 suppresses cancer metastasis by increasing cell-cell adhesion in a calcium-dependent and/or -independent manner.

Because we observed decreases in recycling and exosomal secretion of CD82 proteins upon the mutation of CD82 YXXФ motif, we analyzed the ability of the CD82 YVAA mutant to move in cell migration and invasion experiments, there by determining whether this mutant can still maintain the cell motility-suppressive function of CD82 wild type. Because CD82 may suppress cancer metastasis by increasing cell-cell adhesion in both calcium-dependent and/or-independent manners, we also investigated the effect of aberrant intracellular trafficking of CD82 on cadherin-dependent and -independent cell-cell adhesion.

\subsection{MATERIALS AND METHODS}

\subsubsection{Materials}

The experimental materials used in cell migration and cell-cell adhesion assays include Transwell inserts (Falcon), laminin 111 (Invitrogen), Matrigel (BD Biosciences), 
EDTA (Fisher), ultracentrifuge tubes (Beckman), CD82 mAb TS82b (kindly provided by Dr. Eric Rubenstein of INSERM, France), Transferrin receptor mAb (Molecular Probes), flotillin-1 mAb (BD Transduction Laboratories), Diff-Quik staining kit (Baxter Merz and Dade Behring), CD81 mAb (Abnova), fibronectin (Gibco), EpCAM mAb (Abcam), DMEM (Gibco/Life Technology), Bovine Serum Albumin (Sigma), Fetal Bovine Serum (FBS) (Gibco/Life Technology), and Penicillin and Streptomycin (Gibco/Life Technology).

\subsubsection{Cell motility assays}

Transwell cell migration assay was performed as previously described (Liu et al 2007). Briefly, the transfectant cells were detached with $0.25 \%$ trypsin-EDTA, washed once with PBS, and re-plated onto the Transwell inserts with $8-\mu \mathrm{m}$ pores, which were placed transwell chambers. The underside of the inserts was pre-coated with either fibronectin $(10 \mu \mathrm{g} / \mathrm{ml})$ or laminin $111(10 \mu \mathrm{g} / \mathrm{ml})$ at $4^{\circ} \mathrm{C}$ overnight and then blocked with heat inactivated bovine serum albumin at $37^{\circ}$ for $45 \mathrm{~min}$. Fifty thousand cells suspended in $500 \mu 1$ of $0.1 \%$ heat-inactivated BSA/DMEM were plated onto each insert Five $\mu 1$ of $1 \% \mathrm{FBS} / \mathrm{DMEM}$ were added to the lower wells of the insert.

After incubation at $37^{\circ}$ for $3 \mathrm{hrs}$, the cells that had not migrated through the inserts were removed with cotton swabs and the cells that had migrated to the undersides of the inserts were fixed and stained with Diff-Quick staining kit and counted. Data from independent experiments were pooled and analyzed using a two-tailed student's t-test.

For wound healing assay, the transfectant cells were cultured in 6- or 24-well plates. After the cells formed confluent monolayers, cells were treated with $7.5 \mu \mathrm{g} / \mathrm{ml}$, wounds were created by scratching the monolayer with sterile p-200 pipette tips. The cells were extensively washed with PBS to remove detached cells and the wounded monolayers were replenished with complete DMEM. Wound closure was analyzed using an inverted light microscope at indicated time points. Wound closure or "healing" was quantitated by measuring the width of the wound at different time points and compared between the transfectants statistically.

For invasion assay, briefly, $1 \times 10^{6}$ cells were added to a Transwell insert, which had been coated with Matrigel, in DMEM containing 1\% FBS. The lower wells were loaded with complete DMEM. The cells were incubated at $37^{\circ}$ for $24 \mathrm{hrs}$. The cells that did not invade into Matrigel were discarded while the cells that invaded through Matrigel onto the lower side of the inserts were stained using Diff-Quick solution. The number of the cells that invaded through Matrigel was counted under a light microscope, and the 
results from three independent experiments were quantitated and analyzed using the two-tailed Student's t-test.

\subsubsection{Hanging drop aggregation assay}

The transfectant cells were detached and counted. One thousand cells were resuspended in $30 \mu 1$ of complete or calcium-depleted media, and the single cell suspensions were plated on the inner surface of the lid of a 24-well plate. Each well was filled with PBS, and the lid of the plate was reverted and then placed on the 24-well plate.

The cells were allowed to aggregate, in the hang-drop suspension, over night. After aggregation, the cells were passed through a p-200 tip at least 20 times under mechanical pressure. The cells were photographed under a light microscope and quantified for the numbers of single cells and aggregated cells by manual counting Results are displayed as the ratio of the number of aggregated cells to the number of total cells. The area of aggregates was measured by ImageJ software.

\subsubsection{Cell surface expression}

For flow cytometry, confluent PC3 cells were detached, washed with 1xPBS, and blocked for non-specific binding antibody with $2 \%$ goat serum on ice for 1 hour. Cells were incubated with $1 \mu \mathrm{g} / \mathrm{ml}$ of primary $\mathrm{mAb}$ for EpCAM, $\beta 1$ (TS2/16), or IgG2B for 1 hour followed by PBS washes. The cells were labeled with the appropriate FITC-conjugated secondary antibody for 30 minutes followed by extensive PBS washes. Cell surface expression of the proteins was analyzed using a FACS Calibur flow cyotmeter (BD Biosciences).

\subsubsection{Transmission electron microscopy}

For transmission electron microscopic analysis, the transfectant cells were detached, counted, and seeded at a density of $3 \times 10^{4}$ in transwell inserts. After being cultured in complete media, the cells were fixed with $2.5 \%$ gluteraldehyde in sodium cacodylate buffer. The cells together with insert membrane was then cut into thin sections and stained with $0.3 \%$ potassium ferrocynide in $2.0 \%$ osmium tetroxide and $4.0 \%$ uranyl acetate. The cells were then examined under a transmission electron microscope, and the images were acquired with a digital camera. 


\subsection{RESULTS}

\subsubsection{The mutation of the YXXФ motif does not abolish CD82-mediated inhibition of solitary cell migration}

The mutations of the YXXФ motifs in several transmembrane proteins lead to the defects in protein function and often to disease (Cherqui et al 2001; Shrimpton et al 1997) However, the mutation of the YXXФ motif in CD82 did not affect the motility-suppressive function of CD82 in haptotactic and chemotactic Transwell cell migration assays, as shown in Figure 3-1. Because cells move in an isolated manner in Transwell migration assay, this type of cell migration is considered as solitary cell migration. Cell migration onto either laminin 111 or fibronectin or in complete medium was increased in PC3-CD82 YVAA transfectants. Both migrations of CD82 WT and YVAA cells were inhibited at 3-hour and 24-hour migration time-frame, compared to that of Mock cells.

\subsubsection{The mutation of the YXXФ motif abolished the motility-suppressive function of CD82 in collective cell migration}

We analyzed cell migration using wound healing assay and found that the mutation of the YXXФ motif of CD82 abolished the motility-suppressive function of CD82 in PC3 transfectant cells, as shown in Figure 3-2, and also in Du145 transfectant cells, as shown in Figure 3-3. Upon the mutation of the YХXФ motif, the transfectant cells were able to heal the wound at a significantly faster rate than the CD82 WT transfectant did. These results suggest that cell-cell adhesion and/or contact may be important for the maintenance of CD82 motility-suppressive function.

Due to the increased cell migration observed in the wound healing experiments, we analyzed the invasive potential of the CD82 YVAA transfectant in comparison to the Mock and CD82 WT transfectants to invade through Matrigel, a mixture of extracellular matrices resembling the basement membrane. The results from the invasion assay reveal that the invasive potential of the CD82 YVAA mutant transfectant has been significantly increased, as shown in Figure 3-4, compared to the CD82 WT transfectant, and reached the level of Mock transfectant. It has been reported that CD82 inhibits the invasive potential of cells by increasing the level of tissue inhibitor of metalloproteinase (TIMP), therefore decreasing the levels of matrix metalloproteinanses (MMPs), subsequently resulting in less degradation of the extracellular matrix, and ultimately suppressing cancer metastasis. Because the proteolysis of the extracellular matrix environment determines cell invasiveness, the restored invasive ability upon the YVAA mutation suggests that CD82 inhibits tumor cell invasiveness by altering the production and/or activity of TIMP and MMP. These migration and invasion data, taken together, suggest that the alteration 


\section{3 hour Transwell Cell Migration}

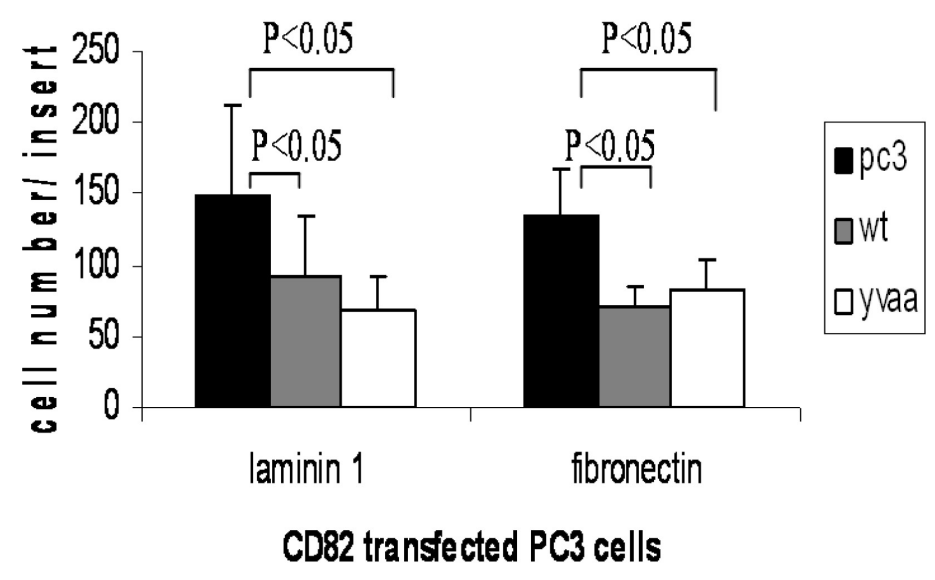

24 hour transwell cell migration

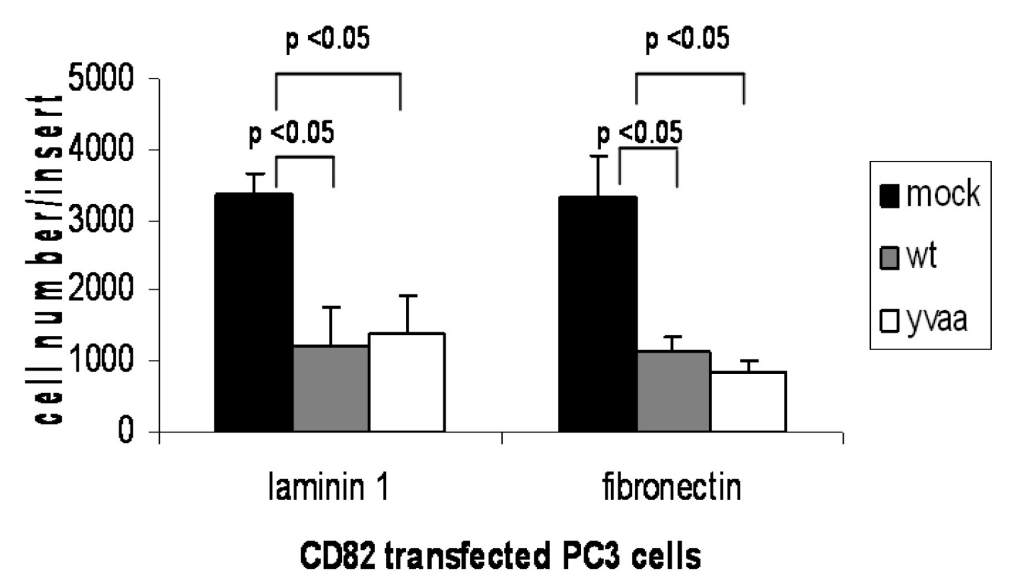

Figure 3-1. Mutation of the YXXФ motif does not disrupt CD82-mediated inhibition of solitary cell movement. The migration of PC3-MOCK, -CD82 WT and -CD82 YVAA transfectant cells was measured by Transwell cell migration assay. Cells were detached, washed with PBS, and seeded at a density of $3.3 \times 10^{3}$ in Transwell insert/filter with $8-\mu \mathrm{m}$ pores. The underside of the insert was precoated with laminin 111 or fibronectin at the concentration of $10 \mu \mathrm{g} / \mathrm{ml}$ at $4^{\circ} \mathrm{C}$ overnight and blocked with $0.1 \%$ heat-inactivated bovine serum albumin at $37^{\circ} \mathrm{C}$ for $30 \mathrm{~min}$. The cells were allowed to migrate for 3 hours (top panel) or 24 hours (bottom panel) at $37^{\circ} \mathrm{C}$ in $5 \% \mathrm{CO}_{2}$. The cells that did not migrate through the insert were removed, and the cells that did migrate through were fixed and stained with Diff-Quick staining kit. 


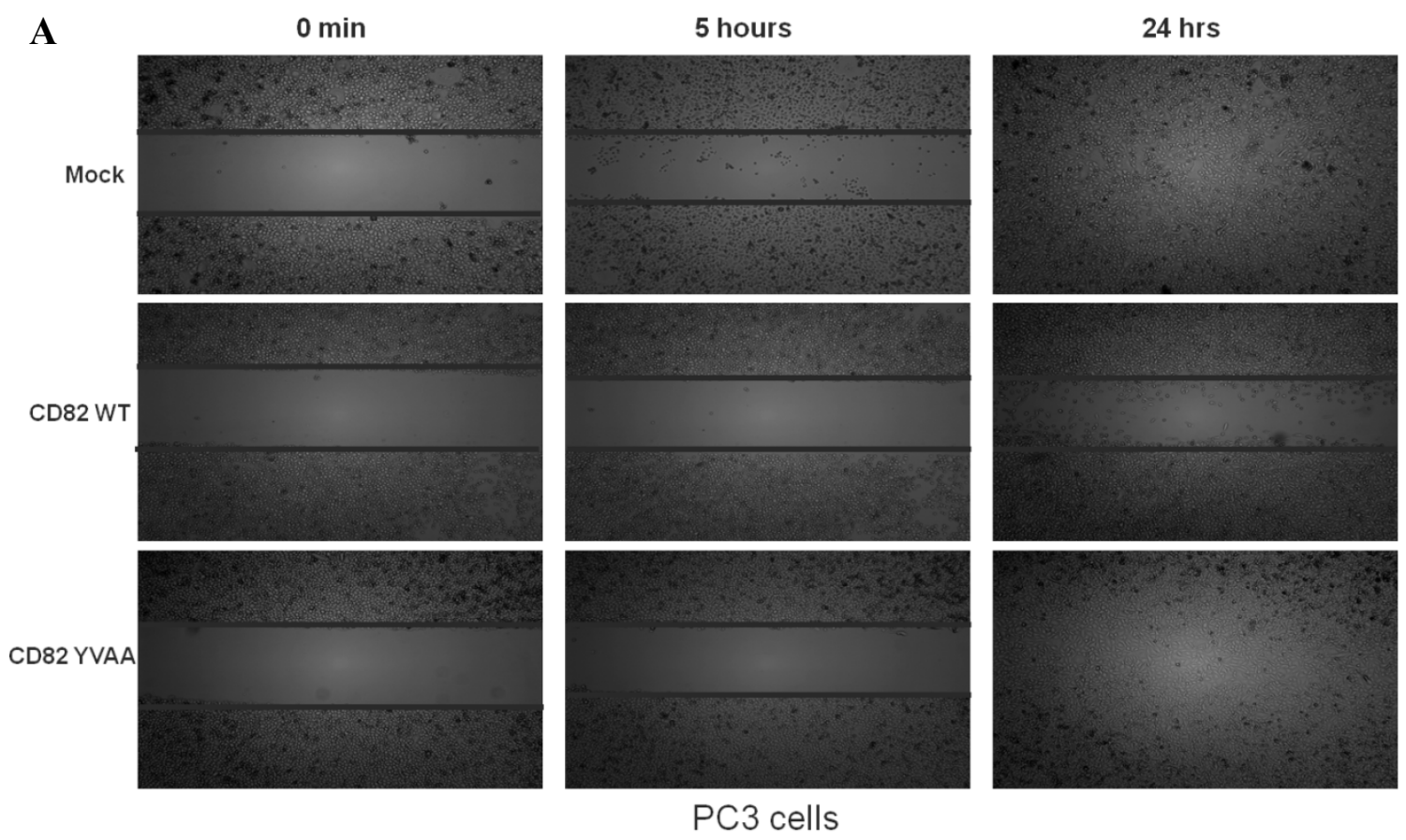

B Rate of cell migration during wound healing

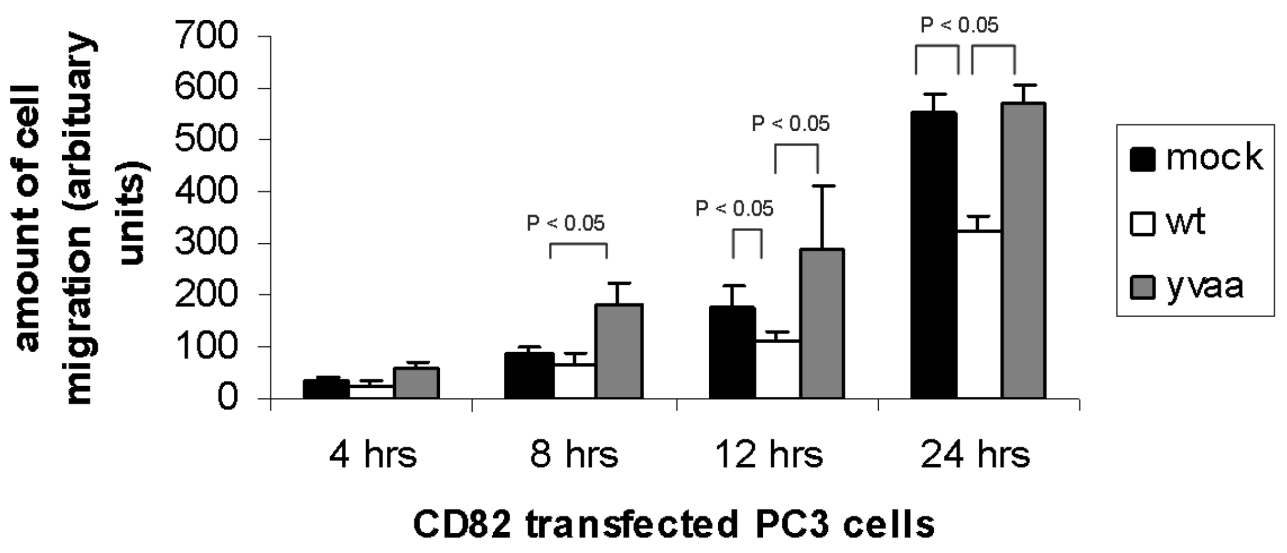

Figure 3-2. The mutation of the YXXФ motif causes a loss of CD82-mediated inhibition in collective cell migration in PC3 cells. The PC3-MOCK, -CD82 WT and -CD82 YVAA transfectant cells were detached, seeded at a density of $1 \times 10^{6}$ in cell culture dishes, and grown to confluency. After the cells formed monolayers, they were treated with $7.5 \mu \mathrm{g} / \mathrm{ml}$ of mitomycin $\mathrm{C}$ to prevent cell growth. The wounds were created by scraping the confluent monolayer with a p- $200 \mu 1$ tip. A, The images of freshly created and healed wounds were captured by using a light microscope attached with a digital camera. B, The wound healing process was quantified at different time point, and the results are depicted as the amount of cell migration over time. 


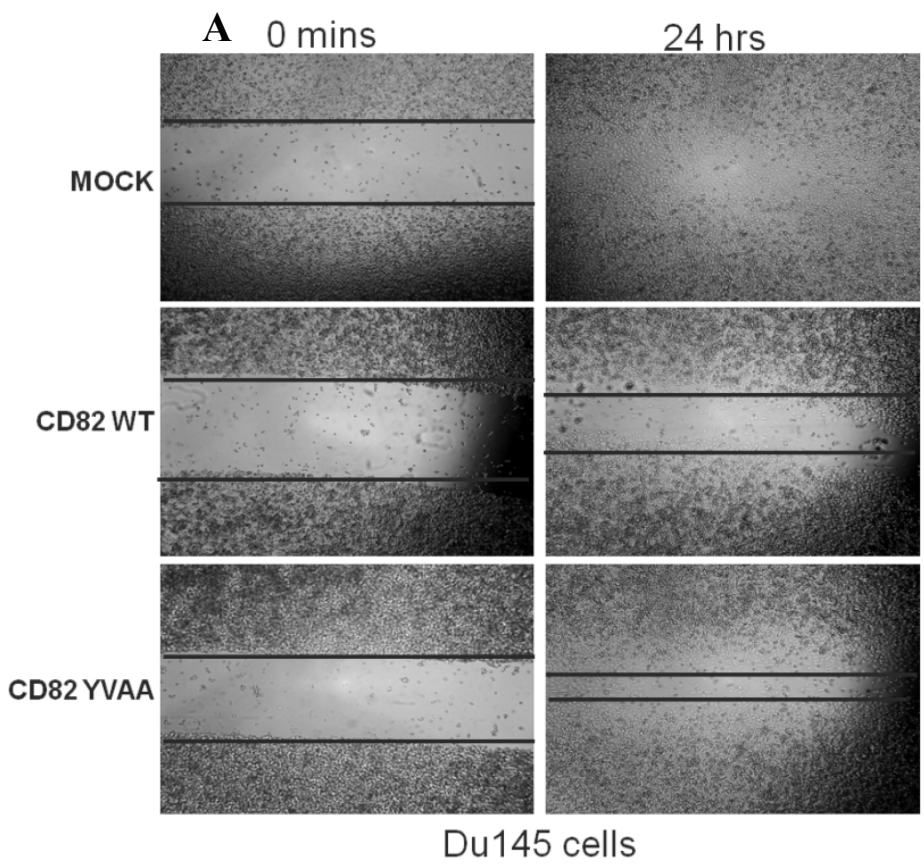

B $24 \mathrm{hr}$ wound healing quantification

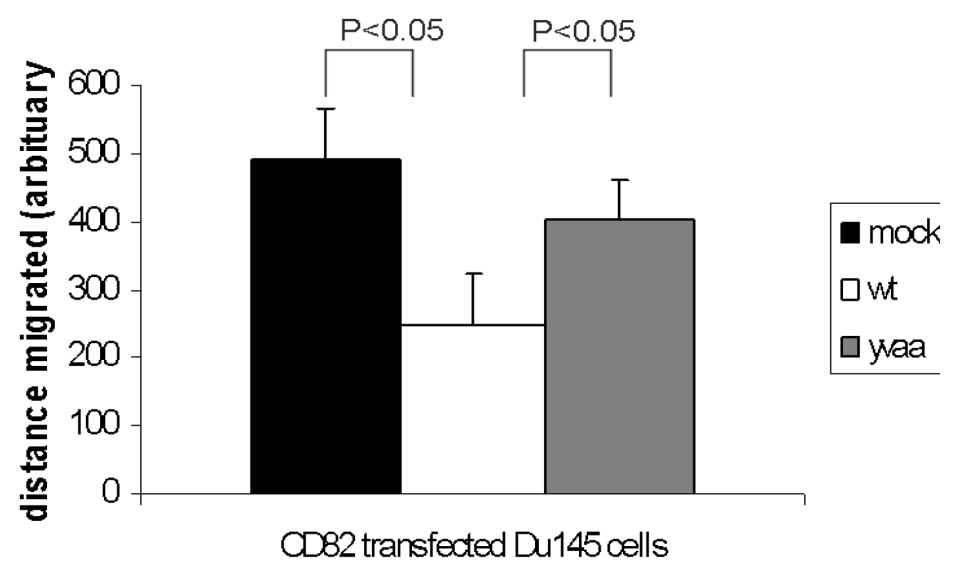

Figure 3-3. The mutation of the YXXФ motif causes a loss of CD82-mediated inhibition in collective cell migration in Du145 cells. The Du145-MOCK, -CD82 WT and -CD82 YVAA transfectant cells were detached, seeded at a density of $1 \times 10^{6}$ in cell culture dishes, and grown to confluence. After the cells formed monolayers, they were treated with $10 \mu \mathrm{g} / \mathrm{ml}$ of mitomycin C to prevent cell growth. The wounds were created by scraping the confluent monolayer with a p-200 $\mu 1$ tip. A, The images of freshly created and healed wounds were captured by using a light microscope attached with a digital camera. B, The wound healing process was quantified at different time point, and the results are depicted as the amount of cell migration over time. 


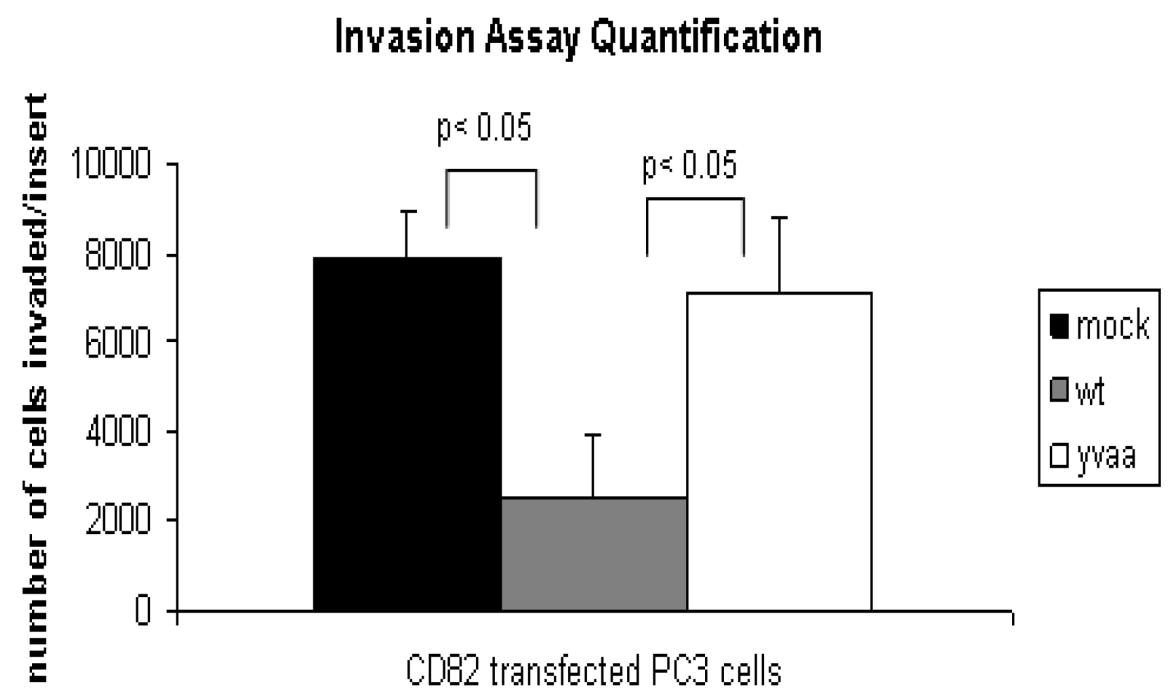

Figure 3-4. The mutation of the YXXФ motif of CD82 attenuates CD82-mediated inhibition of cell invasion. The PC3-MOCK, -CD82 WT and-CD82 YVAA transfectant cells were added to the Transwell inserts, previously coated with Matrigel, in DMEM containing 1\% FBS. The lower wells of the insert were loaded with complete DMEM. The cells were incubated at $37^{\circ}$ for $24 \mathrm{hrs}$. The cells that did not invade through Matrigel were removed, and the cell that invaded through Matrigel onto the lower side of the Transwell inserts were stained using Diff-Quick solution. The number of the cells invaded through Matrigel was counted using a light microscope, and the results from three independent experiments were analyzed statistically using the student's t-test. 
in the intracellular trafficking of CD82, upon mutation of the YXXФ motif, leads to the loss of CD82-mediated suppressive function in collective migration and invasiveness.

\subsubsection{The mutation of the YXXФ motif of CD82 leads to an increase in CD82 mediated cell-cell adhesion}

The over-expression of CD82 leads to an increase in cell-cell adhesion (Jee at al 2008) and has been proposed as one of the mechanisms that CD82 uses to elicit its motility-suppressive function. The wound healing process is regulated not only by cell motility but also by cell-cell adhesion. Since we observed an increase in the wound healing of the CD82 YVAA mutant, we predicted that CD82 YVAA mutant would exhibit an alteration in cell-cell adhesion compared to CD82 WT. Therefore, we compared the strength of calcium-dependent and -independent cell-cell adhesion between the Mock, CD82 WT, and CD82 YVAA transfectants using a hanging drop cell aggregation assay, a well-established method to assess cell-cell adhesiveness.

We evaluated the strength of cell-cell adhesion by quantifying the size of cell aggregates and the number of aggregated cells, as presented in Figure 3-5. To our surprise, the overexpression of CD82 in PC3 cells reduced the level of total cell-cell adhesion, i.e., the sum of calcium-dependent and -independent cell-cell adhesion, after exposure to the stress of shear force. The YVAA mutation attenuated this reduction. We even observed an increase in cell-cell adhesion in PC3-CD82 YVAA transfectant compared to PC3-MOCK transfectant if cell-cell adhesion is quantified as the area of aggregates (see the following paragraph).

We then quantified the difference in total cell-cell adhesion. The histograms in Figure 3-6A represents the quantitative data depicted as the ratio of the number of aggregated cells divided by the total number of cells. After being exposed to shear stress, $90 \%$ of Mock and $94 \%$ of CD82 YVAA transfectant cells remained aggregated together whereas only $74 \%$ of CD 82 WT cells remained aggregated. In Figure 3-6B, the areas of cell aggregates were analyzed by ImageJ software, and the quantification revealed that the average area of each of the CD82 YVAA transfectant aggregate is roughly 25,000 pixels whereas MOCK and CD82 WT trasnfectant aggregate averages roughly 19,000 and 16,500 pixels, respectively. These results indicate that the mutation in the YXX $\Phi$ motif of CD82 restored the reduction in total cell-cell adhesion and may even lead to an increase in cell-cell adhesion compared to Mock cells.

To further explore the mechanism governing CD82-mediated inhibition of cell-cell adhesion, we determined the role of calcium in cell-cell adhesion by removing 


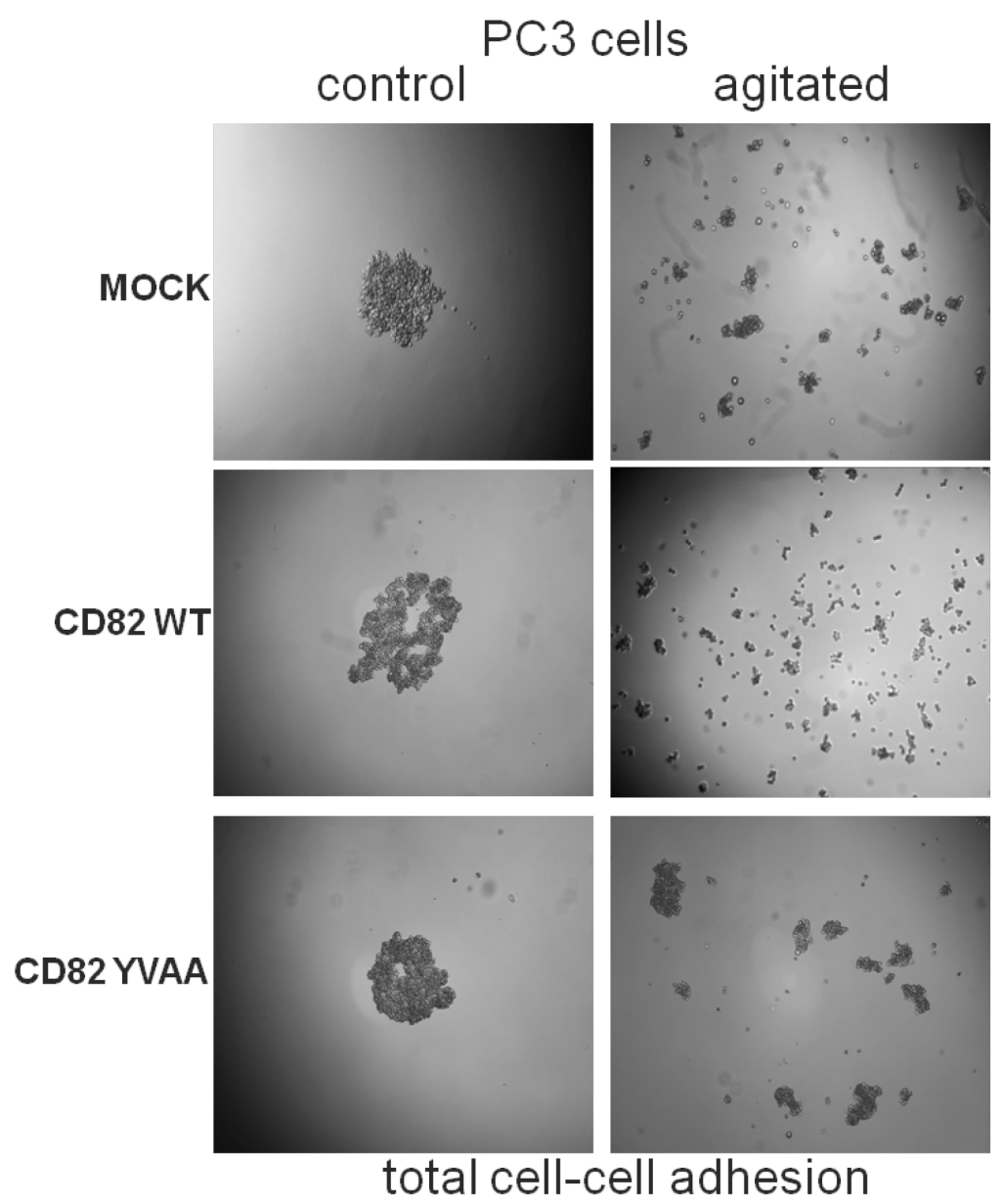

Figure 3-5. The YXXФ mutation attenuates CD82-mediated inhibition of total cell-cell aggregation in PC3 cells. For hanging drop aggregation assay, the PC3-MOCK, -CD82 WT and -CD82 YVAA transfectant cells were detached, resuspended at a density of $2.5 \times 10^{3}$ in $30 \mu \mathrm{l}$ of complete media, seeded on the underside of a 24 -well plate lid, and allowed to aggregate for 24 hours at $37^{\circ} \mathrm{C}$ in $5 \% \mathrm{CO}_{2}$. Aggregated cells were subjected to the shear flow by passing through a $200-\mu 1$ pipetteman tip twenty times. The cell aggregates prior to and after shear stress were photographed using a light microscope attached with a digital camera. 

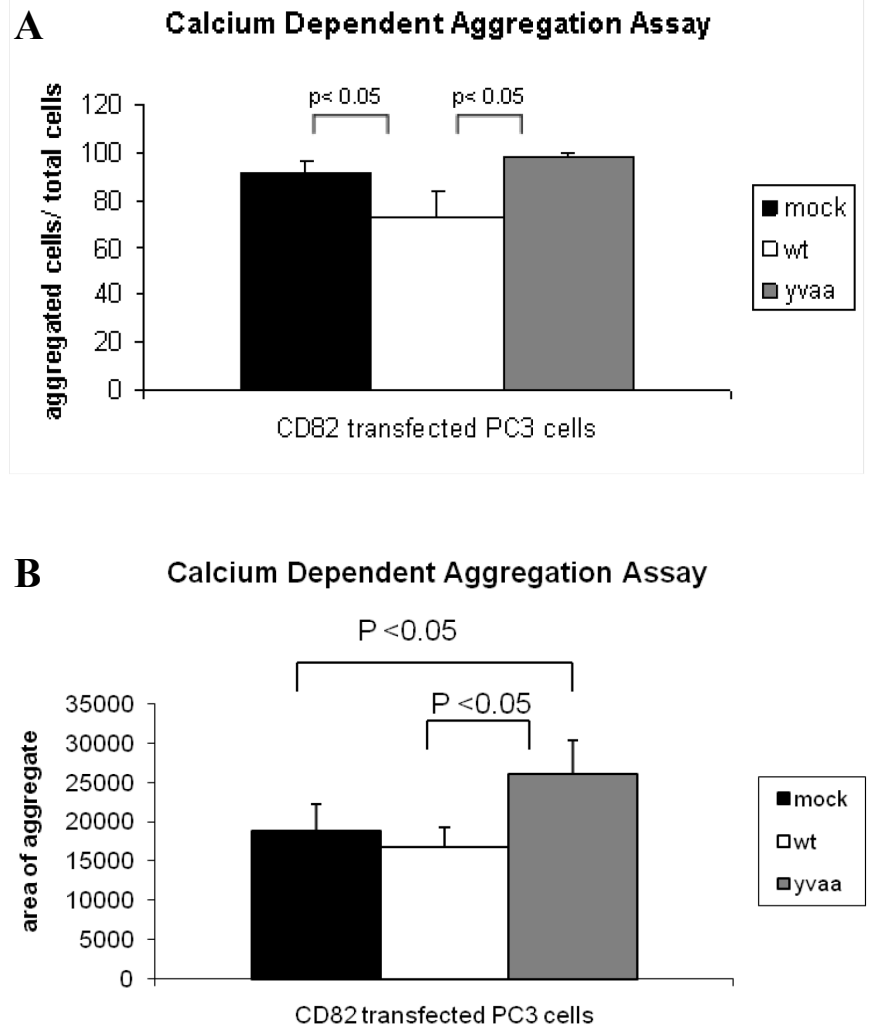

Figure 3-6. Mutation of the YXXФ motif of CD82 attenuates CD82-mediated inhibition of total cell-cell aggregation in PC3 cells. The total cell-cell adhesion of the PC3-MOCK, -CD82 WT and-CD82 YVAA transfectant cells were examined in the hanging drop aggregation assay in complete media as described in Figure 3-5. A, the cell aggregates were quantitated by visually counting the number of aggregated cells and dividing the number of aggregated cells by the number of total input cells. The results are displayed as the percentage of aggregated cells from the total number of cells. $\mathbf{B}$, the surface areas of cell aggregates were quantitated by ImageJ software, and the results are displayed as area of aggregated cells. 
calcium from the medium. We observed, as shown in Figure 3-7, that the difference in cell-cell aggregation between CD82 WT and YVAA transfectants was significantly diminished after shear stress. In Figure 3-7, it was observed that, after shear stress, the aggregates from the PC3-YVAA transfectant were smaller and not tightly aggregated when compared to the aggregates formed in the calcium-dependent aggregation experiment. Moreover, after shear stress, the PC3-YVAA transfectant cells exhibited much more single cells compared to the PC3-Mock and-CD82 WT transfectants in the calcium-independent aggregation experiment.

We then quantified the difference in calcium-independent cell-cell adhesion. The histograms in Figure 3-8A represents the ratio of the number of aggregated cells divided by the number of single cells. We found that there is not a statistically significant difference in terms of the percentage of aggregated cells between PC3-CD82 YVAA and PC3-CD82 WT transfectant cells. Approximately 63\% of CD82 YVAA mutant cells and $72 \%$ of CD82 WT cells remained aggregated after exposure to shear force. The data in Figure 3-8B represents the area of aggregated cells analyzed by image $J$ software and reveals a statically significant difference in the average size of aggregates between the transfectants upon removal of calcium. The average area of each of the CD82 YVAA transfectant aggregate is roughly 12,000 pixels whereas CD82 WT trasnfectant aggregate averages roughly 15,000 pixels and MOCK 21,500. These data indicate that the increased total cell-cell adhesion of CD82 YVAA transfectant is largely calcium dependent.

We also analyzed the cell-cell adhesion in the Du145 transfectants. In contrast to the PC3 transfectants, CD82 overexpression enhanced total cell-cell adhesion in Du145 cells, Figure 3-9A. Similar to the result obtained from PC3 transfectants, CD82 overexpression did not alter the total calcium-independent cell-cell adhesion in Du145 cells, Figure 3-9B. Interestingly, the YVAA mutation markedly increased total cell-cell adhesion in Du145 cells compared to both Mock and CD82-WT transfectant cells, Figure 3-9C, which is consistent with the results from PC3 transfectant cells. But, unlike the effect on PC3 transfectants, the YVAA mutation also markedly increased calcium-independent cell-cell adhesion in Du145 cells.

We also measured the cell surface level of a cell adhesion molecule that is associated with cancer metastasis, EpCAM. As shown in Figure 3-10, we found that, upon mutation of the YXXФ motif of CD82, there is a significant increase in the cell surface levels of EpCAM in the CD82 YVAA mutant compared to the Mock and CD82 WT transfectants. These results suggest that the inefficiency of CD82 YVAA mutant to traffic back to the plasma membrane and be released to the surroundings via exosomes leads to an increase in the expression at the cell surface of cell-cell adhesion proteins, which is normally suppressed by CD82. 


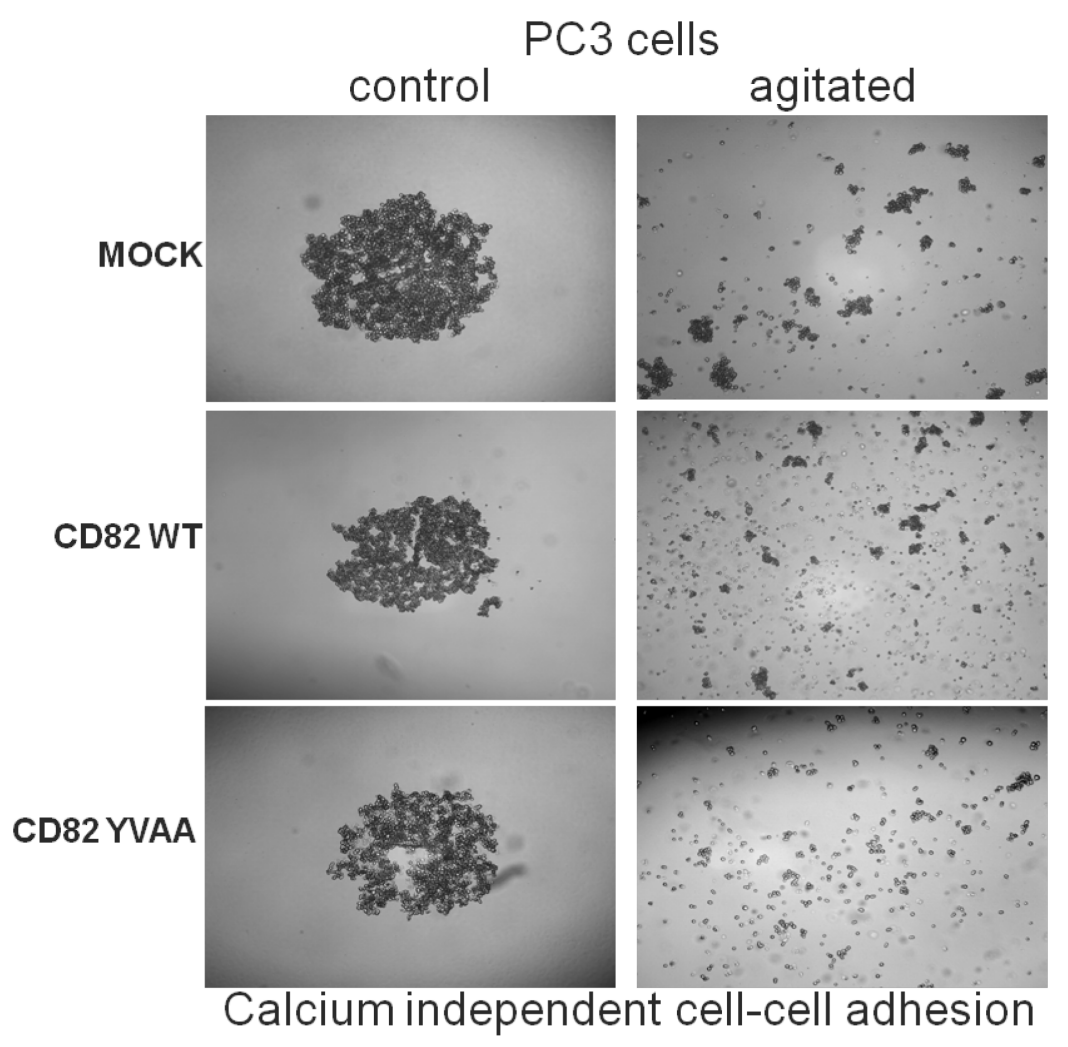

Figure 3-7. The YXXФ mutation does not affect calcium-independent cell-cell aggregation in PC3 cells. For hanging drop aggregation assay, the PC3-MOCK, -CD82 WT and -CD82 YVAA transfectant cells were detached, resuspended in $30 \mu 1$ of calcium-free medium at a density of $2.5 \times 10^{3}$, seeded on the underside of a 24-well plate lid, and allowed to aggregate for $24 \mathrm{~h}$ at $37^{\circ} \mathrm{C}$ in $5 \% \mathrm{CO}_{2}$. Aggregated cells were subjected to the shear stress by passage through a $200-\mu 1$ pipetteman tip twenty times. The cell aggregates prior to and after shear stress were photographed using a light microscope attached with a digital camera. 


\section{A Calcilm Independent Aggregation Assay}

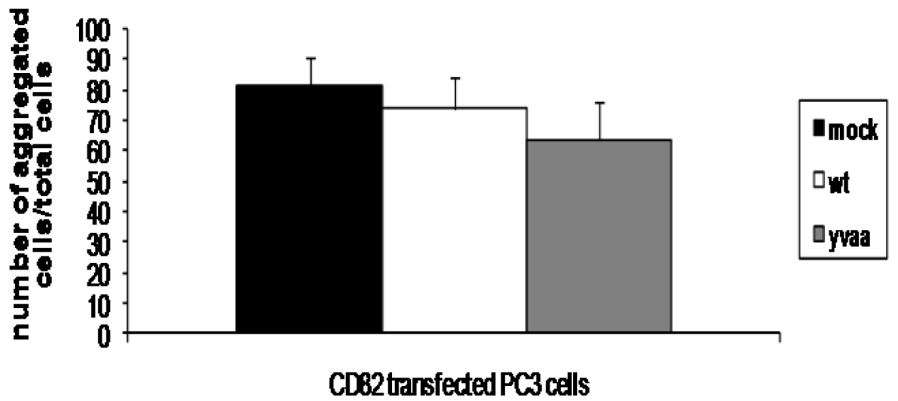

\section{B Calcium Independent Aggregation Assay}

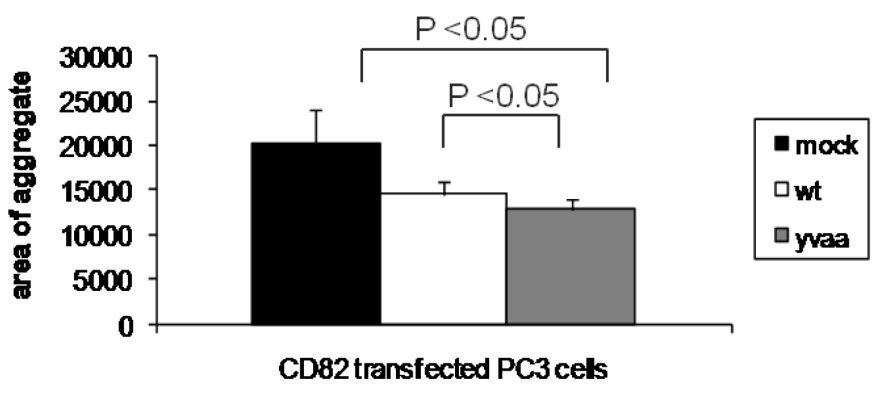

Figure 3-8. The YXXФ mutation decreases calcium-independent cell-cell aggregation in PC3 cells. The total cell-cell adhesion of the PC3-MOCK, -CD82 WT and -CD82 YVAA transfectant cells were examined in the hanging drop aggregation assay in calcium-free media as described in Figure 3-5. A, The cell aggregates were quantitated by visually counting the number of aggregated cells and dividing by the total, input, cell number. The results are displayed as the percentage of aggregated cells divided by the total number of cells. B The surface areas of cell aggregates were quantitated by Image J software, and the results are displayed as area of aggregated cells. 

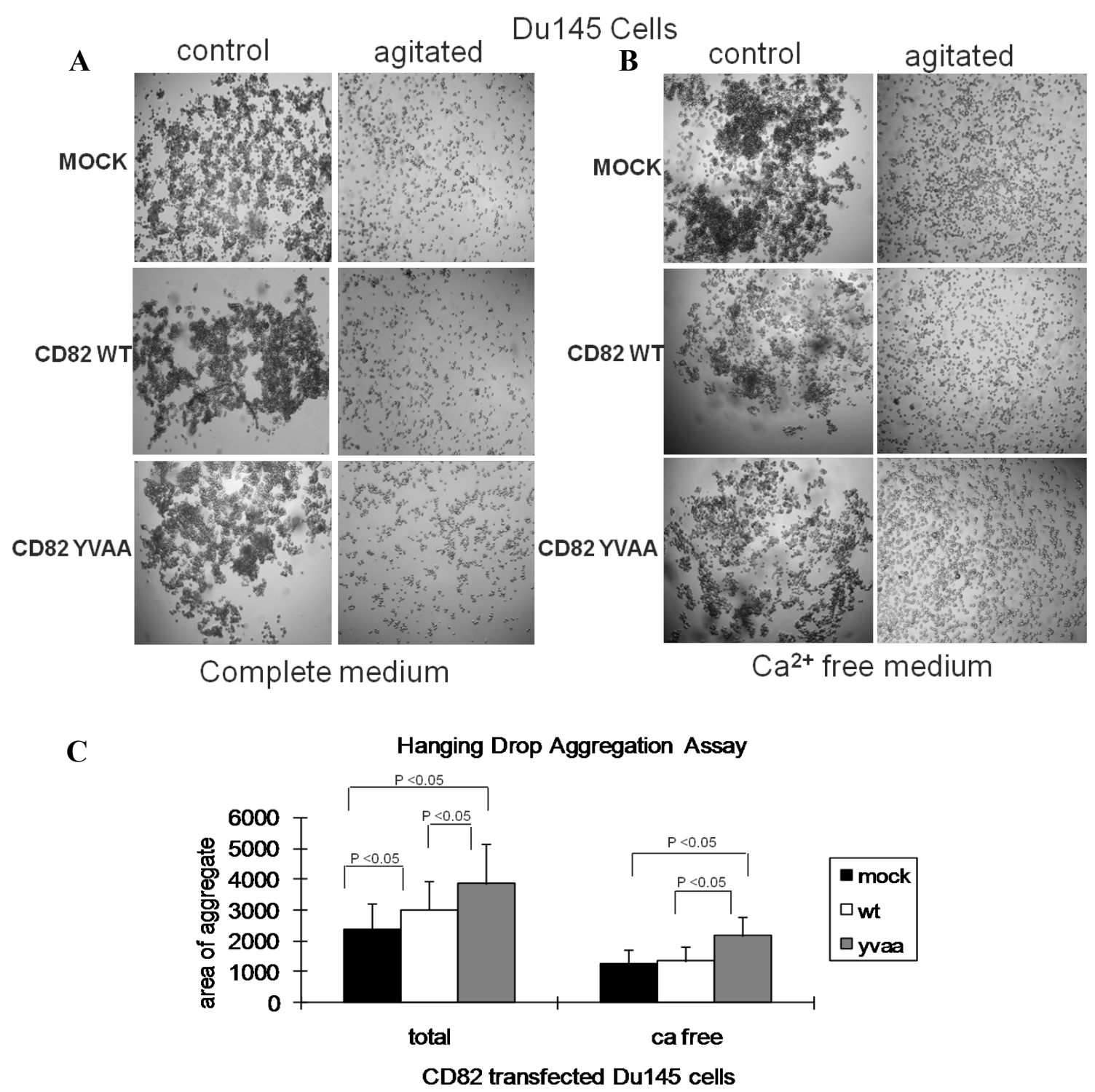

Figure 3-9. The effects of CD82 overexpression and CD82 YVAA mutation on cell-cell aggregation in Du145 cells. For hanging drop aggregation assay, the Du145-MOCK, -CD82 WT and -CD82 YVAA transfectant cells were detached, resuspended at a density of $2.5 \times 10^{3}$ in $30 \mu \mathrm{l}$ of meida, seeded on the underside of a 24-well plate lid, and allowed to aggregate for 24 hours at $37^{\circ} \mathrm{C}$ in $5 \% \mathrm{CO}_{2}$. Aggregated cells were subjected to the shear stress by passage through a $200-\mu 1$ pipetteman tip twenty times. The cell aggregates prior to and after shear stress were photographed using a light microscope attached with a digital camera. A, The hanging drop aggregation assay was performed in complete media. B, The hanging drop aggregation assay was performed in calcium-free media. $\mathbf{C}$, The surface areas of cell aggregates were quantitated by ImageJ software, and the results are displayed as area of aggregated cells. 


\section{Cell surface expression}

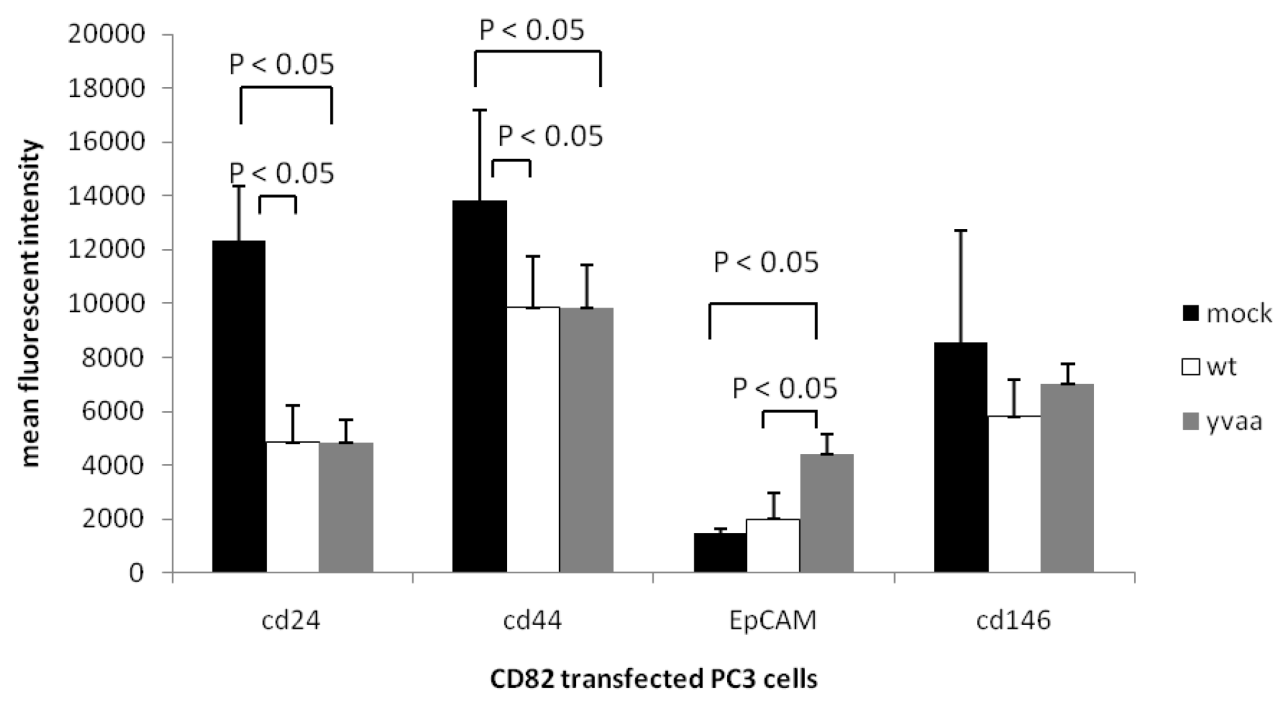

Figure 3-10. The mutation of the YXXФ motif of CD82 leads to an increase in the cell surface expression of the cell adhesion molecule EpCAM. For flow cytometry, confluent PC3 transfectant cells were detached, washed with 1xPBS, and blocked with $2 \%$ goat serum on ice for 1 hour to block non-specific binding. The cells were incubated with $1 \mu \mathrm{g} / \mathrm{ml}$ of primary mAbs, which include murine IgG2b as negative control, $\beta 1$ integrin $\mathrm{mAb}$ TS2/16 as positive control, and EpCAM mAb, at $4^{\circ} \mathrm{C}$ for 1 hour followed by PBS washes. The cells were labeled with FITC conjugated, goat-anti-mouse secondary Ab for 30 min followed by extensive PBS washes. The cell surface expression of the proteins was analyzed using a FACS Calibur flow cyotmeter (BD Biosciences). 


\subsubsection{CD82 YVAA transfectants probably increase desmosome-mediated cell-cell adhesion}

Epithelial cell-cell adhesion is responsible for forming and maintaining the structural integrity of organs and tissues. To further determine the mechanism by which CD82 trafficking regulates cell-cell adhesion; we used transmission electron microscopy to investigate the cell-cell junctions in PC3 transfectants, Figure 3-11. Under the electron microscope, we frequently observed multiple, long, electron-dense cell-cell junctions in PC3-Mock and PC3-CD82 YVAA transfectant cells, Figure 3-11, while the PC3-CD82 WT transfectant cells usually exhibited fewer, shorter, and less electron-dense cell-cell junctions, as shown in Figure 3-11.

The results presented in Figure 3-11A also revealed that the cell-cell junctional structures resemble desmosomes, as indicated by arrows. The length and frequency per cell of desmosomes were analyzed by Image $J$ and visual counting, respectively, and the data are presented in Figure 3-11B and 3-11C. There was an average of 1.7 and 1.5 desmosomes per cell in the MOCK and CD82 YVAA transfectants, respectively, and 0.63 per cell in the CD82 WT transfectants. The Mock and YVAA transfectants exhibited more than a 2 fold increase in the number of desmosomes per cell compared to the CD82 WT transfectant. We also examined the length of desmosomes in these three transfectant, Figure 3-11C, and we found that the average length of desmosomes was $0.45,0.37$, and 0.16 arbitrary units for the CD82 YVAA, MOCK, and WT transfectants, respectively. Together, these data suggest that desmosomes likely take part in the loss of inhibition in calcium-dependent cell-cell adhesion in CD82-YVAA mutant cells and the inhibition in calcium-dependent cell-cell adhesion in CD82 WT cells.

\subsection{SUMMARY}

In this chapter, we focused on the role that the YXXФ motif plays in CD82 motility suppressing function. We observed that the mutation of the YХХФ motif of CD82 leads to the loss of inhibitory activities in collective cell migration and invasion but has no effect on solitary cell migration. These observations imply that CD 82 regulates cell movement through modulating, at least partially, cell-cell adhesion.

We also observed an increase in cell-cell adhesion, which appears to be cadherin-mediated, when the recycling and/or releasing of CD82 become aberrant. This observation implies that the increased cell-cell adhesion in the CD82 YVAA mutant plays a key role in collective cell migration and cell invasion. This result also suggests that the increase in cell-cell adhesion does not necessarily impede cell migration in all circumstances as previously expected. 

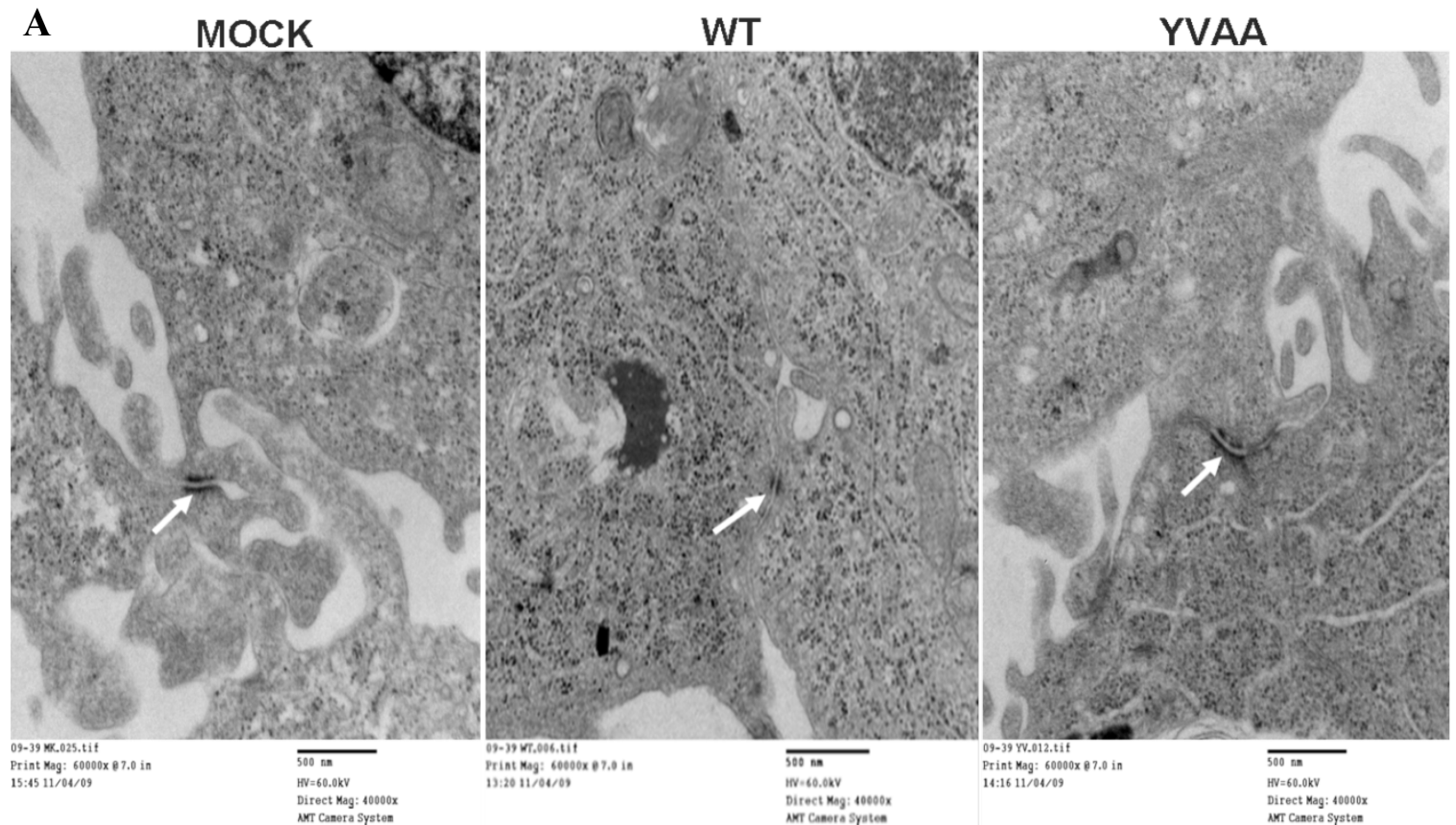

B

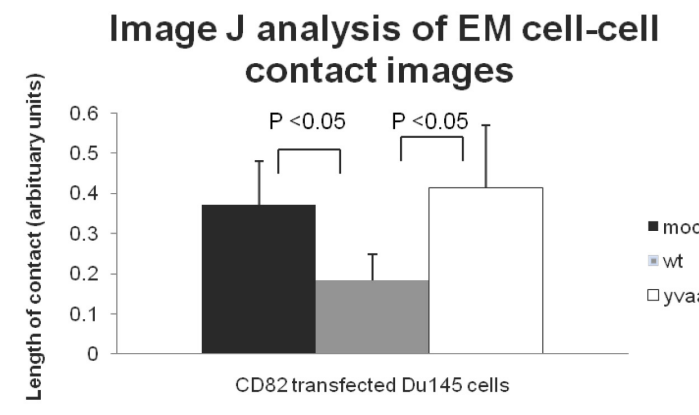

C

Number of contacts per cell

mock

1.72222

wt

yvaa
.631579

1.522727

Figure 3-11. The mutation of the YXXФ motif in CD82 attenuated CD82-dependent reduction in the formation of cell-cell adhesions. For electron microscopic analysis, the PC3 transfectant cells were detached, counted, and seeded at a density of $3 \times 10^{4}$ in Transwell inserts. After being cultured to monolayer, the cells were fixed with $2.5 \%$ gluteraldehyde in sodium cacodylate buffer. The cells together with the filter membrane were then into thin sections and stained with $0.3 \%$ potassium ferrocynide in $2.0 \%$ osmium tetroxinde and $4.0 \%$ uranyl acetate. A, the cells were imaged under a transmission electron microscope connected to a digital camera. B, the length of cell-cell contacts per cell was measured using ImageJ software, and the results are presented as the average length of desmosome. $\mathbf{C}$, The number of desmosomes per cell per section was visually counted, and the data represents the average desmosomes per cell per section by dividing the total numbers of desmosomes with the total number of cells counted ( $\mathrm{n}=30$ cells). 


\section{CHAPTER 4. DISCUSSION}

\subsection{INTRODUCTION}

For the first time, the biological functions of YXXФ motif of CD82 have been characterized. We found that the YXXФ motif of CD82 does not play a crucial role in endocytosis as the YXXФ motifs in other transmembrane proteins such as CD63 and CD151. In addition, the YXXФ motif of CD82 is not required for the targeting of CD82 proteins to the late endosome and/or multivesicular bodies, as evidenced by the equivalent amounts of CD82 wild type and YVAA mutant proteins present in these vesicular compartments. Our studies revealed that the YXXФ motif of CD82 regulates 1) the sorting of CD82 proteins to the lysosomal acidic compartment, 2) the recycling of CD82 from late endosomes back to the plasma membrane, in a Rab 11-dependent manner, and 3) the secreting of CD82 proteins into the extracellular environment, via exosomes.

Most notably, the mutation of the YXXФ motif leads to an abrogation in CD82-mediated inhibition of cell motility, specifically collective cell migration and invasion. We found that the mutation of the YXXФ motif also mediates calcium-dependent cell-cell adhesion and leads to the increase in the cell surface expressions of metastasis-promoting cell-cell adhesion molecules such as EpCAM and I-CAM. Likely, cell-cell adhesion directly contributes to CD82-dependent inhibition of cell motility. In other words, CD82 inhibits cell motility by modulating cell-cell adhesion and the vesicular trafficking of CD82 is involved in this modulation.

It is well established that CD82 is a tumor metastasis suppressor (Liu and Zhang 2006; Miranti 2009; Richardson 2011). However, thus far, it still remains unclear how CD82 elicits its metastasis-suppressive function in vivo and cell motility-inhibitory activity in vitro. There have been several hypotheses proposed to explain how CD82 suppresses metastasis and inhibits cell motility. These include increased cell-cell adhesion, up-regulation of TIMP activity and therefore down-regulation of MMP activity, reorganization of the actin cytoskeleton, preventing protrusion and retraction during cell migration, and interaction with DARC on endothelial cells (Liu and Zhang 2006; Miranti 2009; Richardson 2011).

In this study, we focused on the mechanism by which CD82 inhibits cell migration and cell invasion, from the vesicular trafficking point of view. It has been proposed that CD82 elicits its function indirectly by sequestering other membrane proteins into TEMs and regulating their function (Liu and Zhang, 2006; Miranti, 2009; Richardson 2011). Also, it is well known that the intracellular trafficking of a protein 
could regulate its function. An earlier study from our lab demonstrated that CD82 undergoes sterol lipid-dependent endocytosis and traffics to late endosomal and lysosomal compartments (Xu et al 2009). To our knowledge, there has not been an investigation on the role that intracellular trafficking of CD82 plays in its function. This study sets out to analyze: 1) the role of the YXXФ intracellular trafficking motif in CD82 trafficking and 2) the role of CD82 intracellular is trafficking in CD82-mediated motility-suppressive function.

In the experiments illustrated in Chapter 2, we characterized the role of the YXXФ motif in CD82 trafficking. Based on the results presented in Chapter 3, we defined the role of the CD82 YXXФ motif in the cell motility-suppressive function of CD82. Our current understanding is that proper recycling of CD82 back to the plasma membrane and/or exosomal release of CD82 to the extracellular environment are likely to be important for CD82-mediated inhibition of collective cell migration and invasion. In this chapter we will further discuss our findings/understandings in detail and define the biological significance of these findings.

\subsection{THE YXXФ MOTIF IS NOT ESSENTIAL FOR CD82 INTERNALIZATION}

CD82 is widely expressed in epithelial and endothelial cells (Miranti, 2009). It is found on the plasma membrane, within late endosomes, lysosomes, and in exosomes (Richardson 2011). These observations suggest that CD82 undergoes endocytosis and follows a defined intracellular trafficking route. Understanding the importance of CD82 intracellular trafficking on its function may provide further insight into the mechanism of CD82-mediated inhibition of cell motility.

In this study, we investigated the role of the tyrosine-based sorting motif, YXXФ, in the intracellular trafficking and function of CD82. We generated a mutation in the YXX $\Phi$ motif of CD82, YSKV, by simultaneously replacing the tyrosine and valine residues to alanine, yielding ASKA and obtained the YVAA mutant. Compared to the CD82 WT transfectant, the CD82 YVAA transfectant expressed a similar level of CD82 proteins at the cell surface, suggesting that the YXXФ motif of CD82 is not required for the cell surface expression of CD82 proteins. This YVAA mutation neither caused an alteration in the steady-state distribution of CD82 proteins within cells nor attenuated the internalization of CD82 compared to the CD82 wild type, suggesting that the YXXФ motif of CD82 is not essential for the endocytosis of CD82 proteins.

One of the most noted characteristics of the YXXФ motif function is to rapidly internalize transmembrane proteins and receptors from the plasma membrane (Bonifacino and Dell'Angelica 1999; Bonifacino and Traub 2003; Lam-Yuk-Tseung et al 2005; 
Lam-Yuk-Tseung 2006). Thus, the role of the YXXФ motif in CD82 is inconsistent with the bona fide function of the YXXФ motif, i.e. driving the internalization of transmembrane proteins. For example, the mutation of the tetraspanin CD151 YXXФ motif leads to an accumulation of proteins at the plasma membrane after a 30 to 60 minute pulse-chase endocytosis (Liu et al 2007). However, we found that the colocalization of CD82 proteins with EEA1, a marker of the clathrin-positive early endosomes, became reduced upon YVAA mutation (data not shown), suggesting that CD82 could be internalized through the clathrin pathway although this pathway is not essential for CD82 endocytosis. This observation also suggests that, upon the YVAA mutation, CD82 proteins are driven to the clathrin-independent pathway. Nevertheless, the observations made from this study are consistent with our earlier finding, i.e., the clathrin-dependent pathway is not required for CD82 endocytosis and CD82 typically undergoes clathrin-independent but sterol lipid-dependent endocytosis (Xu et al 2009).

\subsection{THE MUTATION OF THE CD82 YXXФ MOTIF LEADS TO THE ATTENUATION AND ALTERATION IN CD82 RECYCLING}

In addition to the role in internalization, the $\mathrm{YXX} \Phi$ motif has also been implicated in targeting proteins to various intracellular vesicular compartments (Bonifacino and Dell'Angelica 1999; Bonifacino and Traub 2003; Cherqui et al 2001; Lam-Yuk-Tseung et al 2005; Lam-Yuk-Tseung 2006). We observed that the numbers of CD82 YVAA mutant-positive intracellular vesicles were significantly more than those of CD82 WT after prolonged internalization, i.e. 3-hour internalization, suggesting that CD82 YXXФ motif regulates the trafficking step(s) after endocytosis. This observation is similar to previously published reports in which the mutation or truncation of the C-terminal YXXФ motif leads to an accumulation of vesicles within cells due to a defect in recycling (Cherqui et al 2001; Lam-Yuk-Tseung et al 2005; Lam-Yuk-Tseung et al 2006). Indeed, another important characteristic of the YXXФ motif is the delivery of the internalized proteins to the recycling endosomes (Bonifacino and Traub 2003). Hence, the accumulation of CD82 proteins upon the YVAA mutation could result from attenuated recycling.

The recycling data suggests that the function of the YХXФ motif of CD82 is to regulate its intracellular trafficking, specifically recycling. The increase of intracellular CD82 YVAA mutant proteins after 3-hour recycling suggests that some of the internalized CD82 YVAA mutant proteins may traffic to the acidic vesicular compartment and therefore cannot be detected by CD82 mAb, the probe for both endocytosis and recycling assays. The CD82 YVAA mutant proteins may gradually traffic out of the acidic vesicular compartment after the 3 hour incubation at $37{ }^{\circ} \mathrm{C}$ for recycling and subsequently become detectable by CD82 mAb. As a result, more CD82 
staining was found intracellularly in CD82 YVAA transfectant after 3-hour recycling than after endocytosis or 1-hour recycling. Nevertheless, the mutation in the YXXФ motif of CD82 appears to redirect CD82 from the recycling back to the plasma membrane. The data presented in Figure 2-7 represents the average numbers or levels of CD82-positive intracellular vesicles per cell after 1 and 3 hour recycling. In Figure 2-7A, the quantitated data of recycling were obtained from the immunofluorescence images. Figure 2-7B represents the mean fluorescent intensity of the CD82 staining that remained intracellular after recycling. This data suggests that the function of the YXXФ motif of CD82 is to regulate its intracellular trafficking, specifically recycling.

Proteins that are recycled back to the plasma membrane either follow a "fast" internalization and recycling loop in which proteins are typically trafficked back to the plasma membrane within minutes, or a "slow" internalization and recycling loop in which proteins are trafficked back to the plasma membrane within minutes to hours. The Rab4 dependent recycling occurs within minutes and is used primarily by the transferrin receptor and some other proteins whereas the Rab11 dependent recycling can occur within minutes but primarily occurs within hours.

To determine if the intracellulary accumulated CD82 YVAA mutant-positive vesicles resulted from an alteration in the trafficking to the recycling endosomes, we traced CD82 during the early stages of trafficking. An earlier study from our lab demonstrated that CD82 trafficked more readily to the Rab11-positive compartments compared to the Rab4-psotive compartment (Xu et al 2009; our unpublished data). In addition, more colocalization was found between CD82 and Rab11 versus the amount of co-localization of CD82 and Rab4 at steady state (Xu et al 2009; our unpublished data). It was also discovered that, using a dominant-negative Rab11 mutant, CD82 WT proteins were accumulated within Rab 11-positive compartments. Thus, CD82 proteins are more likely recycled back to the plasma membrane through Rab11 recycling endosome pathway. This set of data further corroborates the notion that CD82 recycles slowly. On the contrary, neither the distribution, accumulation, nor the colocalization with Rab11 of the CD82 YVAA mutant-positive vesicles was affected by the dominant-negative mutant of Rab 11 GTPase. It suggests that the mutation of CD82 YXXФ motif disrupts the Rab11-dependent recycling mechanism of CD82 and therefore leads to an accumulation of the CD82 mutant proteins in intracellular vesicles. Hence, the YXXФ motif of CD82 in needed for the Rab 11-dependent recycling of CD82.

The reduced recycling of CD82 YVAA mutant back to the plasma membrane and the unaltered steady-state distribution of this mutant within cells implicate that the CD82 YVAA mutant proteins are recycled back to the plasma membrane through other recycling mechanism(s) such as Rab4 or Rab25 (Caswell et al 2007). The mechanism 
responsible for the recycling of CD82 YVAA mutant remains to be determined in future studies.

\subsection{THE MUTATION OF THE CD82 YXXФ MOTIF DISRUPTS INTRACELLULAR TRAFFICKING OF CD82 AND CAUSES THE MUTATED CD82 PROTEINS TO ACCUMULATE IN LYSOSOMES}

Because CD82 becomes deficient in recycling in the absence of a functional tyrosine-based sorting motif, it is likely that the mutated CD82 proteins will be driven to other intracellular vesicular compartment(s) such as late endosomes and lysosomes. I further analyzed the effect of the mutation of the YХХФ motif on the trafficking of CD82 proteins to other endosomal and/or lysosomal compartments. CD82 has been found in early endosomes, multi-vesicular bodies/late endosomes as well as exosomes (Escola et al 1998; Pelchen-Matthews 2003; Xu et al 2009). These earlier results are consistent with the function of the YXXФ motif in intracellular trafficking; in addition the YXXФ motif was also reported to play a key role in the targeting of proteins to the lysosome for degradation

Both CD82 WT and CD82 YVAA transfectants are localized at the late endosomes to similar extents, as shown in Table 2-1. However, we found that, in comparison to CD82 WT, significantly more CD82 YVAA mutant-positive vesicles belong to the lysosomal compartment (Figure 2-10 and Table 2-1). This observation is atypical for the YXXФ motif due to its lysosomal sorting characteristic/function. If the YXXФ motif of CD82 is required for trafficking CD82 to lysosome, the mutation of the YXXФ motif should lead to the accumulation of the CD82 YVAA mutant-positive vesicles in other intracellular compartments such as the early or late endosomes and result in less accumulation of CD82 in lysosomes. In contrast, more CD82 proteins were accumulated in acidic, lysosomal compartments when the YXXФ motif is mutated. Likely, the CD82 YVAA mutant proteins are redirected to lysosome for degradation because they cannot be properly recycled back to the plasma membrane in a timely fashion (see Section 4. 3). Alternatively, it is also possible that CD82 YVAA mutant proteins become structurally or coformationally aberrant and then destined to lysosomes for degradation. However, the latter is less likely since CD82 YVAA mutant proteins are readily recognized by various CD82 $\mathrm{mAbs}$, can be expressed at the cell surface, still exert inhibition on solitary, and are able to associate with other tetraspanins.

A majority of publications have underlined that one of the main functions of the YXX $\Phi$ motif is to drive the trafficking of the transmembrane proteins to lysosomes for degradation (Bonifacino and Dell'Angelica 1999; Bonifacino and Traub 2003; Cherqui et al 2001; Lam-Yuk-Tseung et al 2005; Lam-Yuk-Tseung 2006). Those transmembrane 
proteins examined in the previous studies usually undergo clathrin-dependent internalization, which is mediated by adaptor proteins, namely the $\mu 2$ subunit of the AP-2 complex. The AP-2 complex plays a key role in initiating transmembrane protein endocytosis, as aforementioned. However, CD82 mainly uses a cholesterol-dependent, lipid raft-mediated, and clathrin-/caveolae-independent mechanism for internalization (Xu et al 2009). Moreover, the C-terminal cytoplasmic domain of CD82 does not interact with the $\mu 2$ subunit or the AP-2 complex of the clathrin machinery in yeast two-hybrid system (Xu et al 2009). Hence, the YХXФ motif of CD82 neither determines the endocytosis of CD82 nor is needed for trafficking CD82 to lysosomes.

It is highly likely that, upon mutation of the YXXФ motif, a default lysosomal trafficking mechanism becomes active by, recognizing the mutated CD82 protein as a protein destined for lysosomal delivery, and traffics CD82 YVAA mutant proteins to the lysosome for degradation. Hence, the CD82 mutant still exhibits the unaltered rates of internalization and trafficking to the late endosome compared to CD82 WT. Also, it is possible that the mutation of CD82 YXX $\Phi$ motif causes CD82 proteins to undergo a different mechanism of internalization and then subsequently a different mechanism of lysosomal targeting. Furthermore, several studies have shown that the heterotetrameric adaptor protein (AP) complexes, localized in endosomes, are important for the targeting of membrane protein to lysosomes. Although CD82 has been shown to have no interaction with the $\mu 2$ subunit of the AP-2 adaptor protein (Xu et al 2009), lines of evidence indicate that the adaptor proteins AP3 and AP4 rather than the AP2 are responsible for lysosomal targeting by binding to the tyrosine-based sorting motif of lysosomal proteins (Aguilar et al 2001; Ohno et al 1998). However, our early study also demonstrated that the C-terminal cytoplasmic domain of CD82 does not interact with the $\mu 3$ and $\mu 4$ subunits of AP3 and AP4 complexes, respectively, in yeast two-hybrid system (Xu et al 2009), consistent with our observation that the YXXФ motif of CD82 is not needed for the lysosomal targeting of CD82. Therefore, further structural-and-functional analysis of the CD82 protein is needed to identify the structural element(s) that determines the lysosomal targeting of CD82.

\subsection{THE MUTATION OF THE CD82 YXXФ REDUCED THE EXOSOMAL SECRETION OF CD82 PROTEINS}

Interestingly, I also found that significantly less CD82 YVAA proteins were released via exosomes compared to the CD82 wild type proteins detected in the exosomal preparation. Notably, the formation and secretion of exosomes per se were not altered by the CD82 YVAA mutation, evidenced by the facts that the equivalent amounts of exosome marker flotillin were found in the exosome preparations from CD82 wild type and CD82 YVAA mutant transfectant cells. These observations actually underline that 
the CD82 YVAA motif facilitates the sorting of CD82 proteins into interluminal vesicles (ILVs) in the multivesicular bodies (MVBs). In addition to CD82, fewer tetraspanin CD81 proteins were also found in exosomes. Because CD81 and CD82 associate with each other, the reduction of CD81 in exosomes probably results from the reduced sorting of CD82 into the exosomes. But CD82 YVAA motif is not required for the formation of interluminal vesicles in MVBs.

Although Rab 27 has been reported to play a key role in the release of exosomes, Rab 11 GTPase is also associated with exosome release (Hsu et al 2010; Savina et al 2005). Because unlike CD82 wild type proteins CD82 YVAA mutant proteins appear to traffic in a Rab11-independent manner, the CD82 YVAA mutant proteins probably cannot be recognized by the exsosomal release mechanism that is regulated by Rab11. As a consequence, fewer CD82 YVAA mutant proteins were released by exosomes compared to CD82 wild type proteins. Hence, the YXXФ motif of CD82 may be needed for the Rab11-dependent exosomal release of CD82.

Taken together, our studies on the roles of the YXXФ motif in CD82 trafficking demonstrated that the function of the YXXФ motif of CD82 is not critical at the internalization step of the intracellular trafficking cascade, consistent with a previous report from our lab, but at the late endosomal trafficking step which subsequently affects downstream trafficking, namely the Rab11-mediated recycling step and the exosomal formation step.

\subsection{THE PROPER INTRACELLULAR TRAFFICKING OF CD82 IS NEEDED FOR CD82-MEDIATED INHIBITION OF CELL MOTILITY}

Based on the hypothesis that the trafficking of CD82 plays an important role in the biological function of CD82, we predicted that the CD82 YVAA mutant no longer carries the metastasis-suppressive and/or motility-inhibitory activities of CD82 due to the decreased recycling back to the plasma membrane and the altered formation of exosomes. The released exosomes may serve as a milieu in the extracellular environment to regulate cell migration.

The main pathological function of CD82 is to inhibit cell movement and cancer metastasis. CD82 has been deemed as a tumor metastasis suppressor due to 1) the loss of expression in metastatic tumors and 2) direct suppression of tumor metastasis in animal models. Metastasis could result from the de-regulation of cell migration. Cell migration is the locomotive process whereby cells move from one location to another. During cell migration, subcellular events including polarization, protrusion and adhesion, translocation of the cell body, and retraction of the rear are involved. These processes are 
coordinated, regulated, and integrated by cell adhesion and signaling proteins such as integrins, cadherins, and immunoglobulin superfamily (IgSF) proteins. Recent studies demonstrated that the trafficking of cell adhesion molecules directly regulate cell movement.

As described in Chapter 3, I analyzed the functional consequence of the mutation of the YXXФ motif on CD82-mediated motility-suppressing functions. I first examined the effect of this mutation on the cell migration-suppressive function of CD82. For the solitary type of cell migration, I observed that the mutation of the YXXФ motif did not affect cell migration compared to CD82 WT. Both CD82 WT and YVAA mutant were able to significantly inhibit solitary cell migration when compared MOCK cells. Because the YVAA mutation attenuates the recycling and exosomal release of CD82, these results suggest that proper recycling and exosomal release of CD82 are not required for CD82dependent inhibition of solitary cell migration. Surprisingly and also interestingly, in wound healing assays, I observed a significant increase in wound closure in the CD82 YVAA mutant transfectant compared to that of the CD82 WT transfectant. The rate of wound closure of the CD82 YVAA transfectants was almost identical to that of the MOCK transfectants. Because cells migrate as cohorts during the wound healing process and this assay specifies collective cell migration, these results suggest that the YXXФ motif is needed for CD82-mediated inhibition of collective cell migration. Because cell-cell adhesion is heavily involved in collective migration, this result also implicates that the CD82 trafficking steps determined or influenced by its YХХФ motif could regulate cell-cell adhesion.

These results further suggest that 1) vesicular trafficking is involved differentially in solitary and collective cell migrations and 2) the subcellular mechanism that cells use for solitary migration is quite different from the one that cells use for collective cell migration. The conclusions from these observations include that 1) the migration properties for single cell migration are different from collective cell migration, 2) the intracellular trafficking of CD82 such as recycling and exosomal release likely regulates cell-cell adhesion, and 3) the altered cell-cell adhesion caused by the aberrant CD82 trafficking likely results in the abolishment of motility-inhibitory activity of CD82.

In both collective and solitary cell movement, the cells must become anterior-posterior or front-rear polarized in order to migrate; otherwise the cells will remain stationary. In the Transwell cell migration assay, the cells are influenced by chemo-hapotactic attractants, which induce the basic subcellular events of cell migration such as membrane protrusions, interaction with extracellular matrix proteins, and actincytoskeletal arrangement, to migrate in a defined direction. This assay measures integrin-mediated and growth factor-stimulated migration onto extracellular matrix 
proteins of isolated cells. CD82 associates with integrins, interferes with integrin mediated cell- matrix adhesion, regulates the trafficking of its associated integrins, and modulates integrin-initiated signaling events (He et al 2005; Zhang et al 2003). In addition, CD82 attenuates growth factor signaling such as EGFR and c-Met signaling (Odintsova et al 2000; Odintsova et al 2003; Sridhar and Miranti 2006; Takahashi et al 2007). At the subcellular level, CD82 inhibits the formation of lamellipodia at the leading edge of migrating cells (Liu and Zhang 2006). CD82 has also been implicated to repress the trailing edge retraction process of migrating cells by re-organizing the actin cytoskeleton, rendering the cells into less motile state, and thereby decreasing the rate of cell migration. Collectively, these results suggest that both CD82 WT and CD82 YVAA mutant are able to 1) associate with integrins with similar affinities, 2) regulate integrin trafficking in a similar manner, and 3) exhibit defects in protrusion and retraction during cell migration.

In the wound healing assay, the cells are free to migrate in a random direction but typically migrate together in a cell-cell adhesion dependent manner. Although many of the biological properties of Transwell cell migration overlap with the collective cell migration observed in wound healing, there are several key differences. In collective cell migration, in addition to regulatory factors such as cell-cell adhesion, factors such as growth factors, chemokines, and extracellular matrix-cell adhesion directly influences migration. For example, collectively migrating cells also 1) interact with each other thereby maintaining cell-cell contacts and junctions, 2) coordinate the pushing and pulling forces necessary for the maintenance of cell-cell junctions and continuity of cell migration, and 3) have the potential to provide each individual cell with spatial input as they occupy discrete places and sense the gradients of soluble factors and matrices in the environment differently (Friedl and Gilmour 2009; Gaggioli et al 2007; Rørth et al 2011).

Compared to the motility of CD82 WT, the increased collective cell migration observed in the CD82 YVAA mutant is highly likely to be the direct results of fewer CD82 and its associated/regulated proteins being 1) recycled back to the plasma membrane and/or 2) secreted via exosomes to the extracellular environment. Exosomes are secreted by almost all kinds of cells, and their secretion has been associated with immune response, virus incorporation, tumorigenesis, and cancer metastasis. In terms of cancer metastasis, exosomes primarily mediate the release of a wide variety of proteins, lipids, mRNAs, and micro RNAs, which can be uptaken by neighboring cells and can therefore transmit pro-metastatic signals (Shen et al 2011). For collective cell migration, the exosomal release may cause the secretion of proteins that either are required for or promote cell-cell adhesion from the cells and therefore lead to the reduction in collective cell migration. Because the mutation of CD82 YXXФ motif results in less exosomal 
release of CD82 and probably also its associated proteins such as CD81, more cell-cell adhesion proteins needed for collective cell migration may become available in the YVAA mutant cells, and consequently the collective cell migration of the mutant is elevated compared to the CD82 wild type cells.

Although cells invade through three dimensional (3D) matrix environment in an isolated manner, CD82 YVAA mutant no longer exerts the invasion-inhibitory activity as CD82 wild type does, in contrast to the effect of this mutant on solitary cell migration. The altered cell-cell adhesion in CD82 YVAA mutant, which contributes to the altered collective cell migration as described above, is unlikely involved in the loss of invasion-inhibitory activity of this mutant. The only difference between Tranwell cell migration and invasion assays is that the tumor cells are surrounded by matrices in invasion assay instead of diffusible media in the solitary migration assay. Probably, the exosomes released from the cells are accumulated in the cell surroundings and serve as a tool to communicate between the isolated cells during invasion process. The exosomes may play roles in coordinating cell invasive behaviors, and the altered composition in the exosomes due to the YVAA mutation may attenuate the invasiveness of CD82.

\subsection{THE MUTATION OF THE YXXФ MOTIF IN CD82 ALTERS CELL-CELL ADHESION IN TUMOR CELLS}

Because the mutation of CD82 YXXФ motif selectively alters CD82-mediated inhibition of collective cell migration, I analyzed the cell-cell adhesive strength of the CD82 YVAA transfectant. Cell-cell adhesion is mediated by cell adhesion proteins such as cadherins, immunoglobulin superfamily (IgSF) members, and sometimes integrins, all of which are directly or indirectly connected to the actin cytoskeleton and/or intermediate filament and thereby provide the mechanical strength at cell-cell contacts (Friedl and Gilmour 2009). It has been previously shown that the over-expression of CD82 leads to an increase in cell-cell adhesion and a decrease in cell migration (Abe et al 2008; Jackson et al 2000; Liu et al 2003; Shibagaki et al 1999). Indeed, I found that the total cell-cell adhesion was enhanced upon CD82 expression in Du145 cells. However, in our study, the total cell-cell adhesion was reduced upon CD82 expression in PC3 cells. In addition, I observed that the mutation of the YXXФ motif of CD82 leads to an increase in total cellcell adhesion in the CD82 YVAA transfectants compared to the CD82 WT transfectants in both PC3 and Du145 cells.

The study from Abe et al suggested that CD82-mediated enhancement of cell-cell adhesion was calcium-dependent and CD82 upregulated cell-cell adhesion by stabilizing the E-cadherin- $\beta$-catenin complex (Abe et al 2008). I further analyzed the effect of the mutation of the YXXФ motif on cell-cell adhesion in the absence of calcium. The 
removal of calcium reduced the cell-cell adhesiveness of the CD82 YVAA mutant to the levels of MOCK and CD82WT transfectants in PC3 cells. The removal of calcium had minimal effect on the cell-cell adhesion in CD82 WT and MOCK cells. Thus, the increase in the total cell-cell adhesion, observed in PC3-CD82 YVAA transfectant, appear to be calcium-mediated. But this conclusion may not apply Du145 cells because Du145-CD82 YVAA transfectant still exhibited the elevated cell-cell adhesiveness in the absence of calcium, nevertheless, the YVAA mutation apparently upregulates cell-cell adhesion in some cell types.

The altered cell-cell adhesion may result from the alteration in the expression levels or function of cell-cell adhesion molecules or structural integrity of cell-cell junctions. To further characterize the cell-cell adhesive/adhesion properties of these transfectants, I performed TEM analysis on the transfectant cells that reached confluence and formed well-developed cell-cell contacts. I discovered that the YXXФ motif mutation of CD82 led to increases in the number, length, and density of the cell-cell junctions, in the PC3-CD82 YVAA transfectant cells compared to the PC3-CD82 WT transfectant cells. There was no difference between the MOCK and CD82 YVAA transfectants. This morphological change is consistent with the results from cell-cell adhesion assay in PC3 cells described above.

Invasive tumor cells express cell-cell adhesion molecules including E-, N-, and desmosomal cadherins, tight junction proteins, and gap junction proteins. Based on the morphological characteristics of these cell-cell junctional structures, I predict that they are desmosomes or desmosome-like junctions. The desmosomes are calcium-mediated junctional structures. Desmosomal cadherins are the cell-cell adhesion molecules responsible for firmly binding neighboring cells together and resisting shear forces and mechanical insults. Thus, the electron microscopic observations support the conclusion that the mutation of CD82 sorting motif selectively or mainly upregulates calciumdependent cell-cell adhesion in PC3 cells. Meanwhile, this electron microscopic result does not exclude the changes in other cell-cell adhesion mechanisms that do not form cell-cell junctional structures upon the YVAA mutation.

To determine whether the altered cell-cell adhesion caused by the changes in the expression levels of cell-cell adhesion molecules, I also analyzed the cell surface expression of the most abundant, widely expressed, and well-studied cadherin, Ecadherin. Abe et al has previously shown that the increase in E-cadherin-mediated cell-cell adhesion caused by CD82 overexpression is a direct result of the physical association between CD82-E-cadherin and that this association strengthens the E-cadherin-mediated intercellular adhesion (Abe et al 2008). Deregulation of E-cadherin mediated cell-cell adhesion is associated with increased cancer metastasis and is therefore 
considered as a tumor metastasis suppressor (Strumane et al 2004). However, I did not find significant differences in the cell surface expression levels of E-cadherin between any of the transfectants in both PC3 and Du145 cells (data not shown).

Typically, the increased cell-cell adhesion in epithelial cells, with the exception of collective cell migration such as wound healing, results in the decrease in cell migration. The increased surface expression of cell-cell adhesion proteins usually leads to enhanced cell-cell adhesion. Interestingly, I found that the cell surface expression of IgSF cell-cell adhesion proteins such as I-CAM and EpCAM are upregulated upon the mutation of the YXXФ motif of CD82 in PC3 cells. Both I-CAM and EpCAM are associated with increased cancer metastasis (Brooks et al 2008; Gostner et al 2011; Hayes and Seigel 2009; Koprowski et al 1979; Lin et al 2006; Osta et al 2004; Trzpis et al 2007; Wang et al 2006;). EpCAM is expressed on prostate cancer cells and its expression is upregulated in the absence of CD82 expression (Mohan et al 2007; Poczatek et al 1999). IgSF proteins mediate calcium-independent cell-cell adhesion. The calcium-independent cell-cell adhesion was indeed upregulated in the Du145 transfectant but remained unaltered in the PC3 transfectant upon the YVAA mutation in CD82. Thus, the molecules responsible for the YVAA mutation-induced increase of cell-cell adhesion still remain to be determined. Nevertheless, the altered cell-cell adhesion upon YVAA mutation supports the notion that proper trafficking of CD82 to i) the plasma membrane via recycling endosomes and ii) the extracellular environment via exosomes are critical steps in the maintenance of CD82-mediated suppression of cell motility and likely tumor metastasis as well.

\subsection{CONCLUSION}

Taken together, this data suggests that the YXXФ motif of CD82 is not important for the regulation of internalization but that it is important for recycling and exosomal release. Functionally, this motif is important for the maintenance of CD82 suppressive regulation of collective cell migration and invasion. I predict that the mutation of the YXXФ motif causes CD82 to use a different intracellular trafficking route, and therefore CD82 YVAA mutant exhibits different rates and/or courses in recycling, lysosomal targeting, and exosomal release from CD82 WT. An alteration in the intracellular trafficking indeed leads to the changes in the functions related to cell motility. For example, it can be explained that, due to an accumulation of CD82 YVAA mutant proteins within the cells, fewer CD82 YVAA mutant proteins are dynamically presented at the cell surface. It appears that such dynamic presentation of CD82 to the cell surface during cell movement is important for the motility-inhibitory activity of CD82.

CD82 regulates cell-cell adhesion in both calcium dependent and independent manners. With less and/or slower return of CD82 proteins back to the cell surface in the 
YVAA transfectant cells, metastasis-promoting cell adhesion molecules such as EpCAM, which is down-regulated by CD82 WT expression, are now capable of functioning. Similarly, more calcium-dependent cell-cell adhesion proteins become functional in CD82 YVAA transfectant cells, leading to the increase in cell-cell adhesion. Consequently, there is an increase in collective cell migration. This study has 1) shed invaluable insight on the mechanism of CD82-mediated inhibition of tumor cell migration and invasion, 2) revealed an unexpected, function of the YXXФ motif, i.e., exosomal release, and 3) has further strengthened the notion that intracellular trafficking regulates transmembrane protein function.

\subsection{FUTURE COURSE}

It can also be hypothesized that, due to the accumulation of CD82 YVAA mutant proteins within the cells the CD82 YVAA mutant proteins cannot be dynamically presented to the cell periphery or surface, supported by the results from recycling and exosome release, to elicit the suppressive function in cell movement. Further investigation of the functional importance of exosomes in CD82-mediated inhibition of cell migration is needed. The first question that remains to be addresses is whether or not the use of purified isolated exosomes from the CD82 WT transfectant is sufficient to inhibit cell migration in the MOCK and CD82 YVAA mutant transfectants. The second issue that needs to be addressed is whether there is an alteration in the receptors, proteins, mRNAs or microRNAs released in the transfectatns. The last question that needs to be addressed is whether a mutation of Rab 11 GTPase, a mediator of CD82 recycling, will render CD82 WT non-functional. Answers to these remaining questions will provide much needed insight into the mechanistic view of CD82 function, Rab 11 function in recycling and exosome secretion, and the influence of exosomal release on neighboring cell behaviors.

Of note, I also observed the expressions of cell adhesion and cell surface proteins such as CD44, CD24, integrin $\alpha 2, \mathrm{CD} 146$ are altered upon the CD82 expressions in PC3 and Du145 cells. Some of these proteins such as CD44, CD24, and integrin $\alpha 2$ are the markers of tumor initiating cells or cancer stem cells. It raised an interesting question: whether CD82 suppressing tumor progression and metastasis by reducing tumor initiating cells or changing the adhesion and migration abilities of tumor initiating cells. This remains to be determined in future studies. 


\section{LIST OF REFERENCES}

Abe M et al. (2008). A novel function of CD82/KAI1 on E-cadherin-mediated homophillic cellular adhesion of cancer cells. Cancer Lett. 266:163-70.

Adachi M et al. (1996). Correlation of KAI1/CD82 gene expression with good prognosis in patients with non-small cell lung cancer. Cancer Res. 56:1751-5.

Aguilar R et al. (2001). Signal-binding specificity of the mu4 subunit of the adaptor protein complex AP-4. JBC. 276(16):13145-52.

Alberts B, Johnson A, Lewis J, et al. (2002). Molecular Biology of the Cell. 4th edition. New York: Garland Science.

Artavanis-Tsakonas S et al. (2011). The tetraspanin CD82 is specifically recruited to fungal and bacterial phagosomes prior to acidification. Infect Immun. 79(3):1098-106

Bari R et al. (2009). Transmembrane interactions are needed for KAI1/CD82 mediated suppression of cancer invasion and metastasis. Am J Pathol. 174(2):647-60.

Berditchevski F. (2001). Complexes of tetraspanins with integrins: more than meets the eye. JCS. 114:4143-51.

Berditchevski F, Odintsova, E. (2007). Tetraspanins as regulators of protein trafficking. Traffic. 8:89-96.

Berx G, Becker K, Hofler H, van Roy F. (2008). Mutations of the human E-cadherin (CDH1) gene. Hum Mutagenesis. 12:226-37.

Birchmeier W, Behrens J. (1994). Cadherin expression in carcinomas: role in the formation of cell junctions and the prevention of invasiveness. Biochim Biophys Acta. 1198:11-26.

Bonifacino J, Dell'Angelica E. (1999). Molecular bases for the recognition of tyrosine-based sorting signals. JCB. 145(5):923-6.

Bonifacino J, Traub L. (2003). Signals for sorting of transmembrane proteins to endosomes and lysosomes. Annu Rev Biochem. 73:395-447.

Bouras T, Frauman A. (1999). Expression of the prostate cancer metastasis suppressor gene KAI1 in primary prostate cancers: a biphasic relationship with tumour grade. J Pathol 188(4):382-8. 
Briese J et al. (2008). Correlations between reduced expression of the metastasis suppressor gene KAI-1 and accumulation of p53 in uterine carcinomas and sarcomas. Virchows Arch. 453(1):89-96.

Brooks K, Coleman E, Vitetta E. (2008). The antitumor activity of an anti-CD54 antibody in SCID mice xenografted with human breast, prostate, non-small lung, and pancreatic tumor cell lines. Int J Cancer. 123(10): 2438-45.

Brown D, Benton S. (2000). Sorting proteins to their target membranes. Kidney Itn. 57:816-24.

Buschow S et al. (2009). MHC II in dendritic cells is targeted to lysosomes or T Cell-induced exosomes via distinct multivesicular body pathways. Traffic. 10(10):1528-42.

Canal $\mathrm{M}$ et al. (2010). Quantitative in vivo imaging of the effects of inhibiting integrin signaling via Src and FAK on cancer cell movement: effects on E-cadherin. Dynamics of Cancer Res. 70:9413-22.

Chairoungdua A, Smith D, Pochard P, Hull M, Caplan M. (2010). Exosome release of $\beta$-catenin: a novel mechanism that antagonizes Wnt signaling. JBC. 190(6): 107991.

Chen Z et al. (2004). CD82, and CD63 in thyroid cancer. Int J Mol Med. 14(4):517-27.

Cherqui S, Kalatzis V, Trugnan G, Antignac C. (2001). The targeting of cystinosin to the lysosomal membrane requires a tyrosine-based signal and a novel sorting motif. JBC. 276(16):13314-21.

Christgen $\mathrm{M}$ et al. (2008). KAI1/CD82 is a novel target of estrogen receptor-mediated gene repression and downregulated in primary human breast cancer. Int $\mathrm{J}$ Cancer. $15: 123(10): 2239-46$.

Christgen M et al. (2009). Expression of CD82 in distant metastases from estrogenreceptor negative breast cancer. Cancer Sci. 100(9):1767-71.

Conner S, Schmid S. (2003). Regulated portals of entry into the cell. Nature. 422:37-44.

Delcayre A et al. (2005). Exosome display technology: applications to the development of new diagnostics and therapeutics. Blood Cells Mol Dis. 35(2):158-68.

Dong J et al. (1995). KAI1, a metastasis suppressor gene for prostate cancer on human chromosome 11p11.2. Science. 268(5212):884-6. 
Dong J et al. (1996). Down regulation of the KAI1 metastasis suppressor gene during the progression of human prostatic cancer infrequently involves gene mutations or allelic loss. Cancer Res. 56(19):4387-90.

Dong J et al. (1997). Genomic organization of the human KAI1 metastasis-suppressor gene. Genomics. 41:25-32.

Duijvesz D, Luider T, Bangma CH, Jenster G. (2011). Exosomes as biomarker treasure chests for prostate cancer. Eur Urol. 59(5):823-31.

Escola J et al. (1998). Selective enrichment of tetraspan proteins on the internal vesicles of multivesicular endosomes and on exosomes secreted by human b-lymphocytes. JBC. 273(32):20121-7.

Farhadieh R et al. (2004). Down-regulation of KAI1/CD82 protein expression in oral cancer correlates with reduced disease free survival and overall patient survival. Cancer Lett. 213(1):91-8.

Felder S et al. (1990).Kinase activity controls the sorting of the epidermal growth factor receptor within the multivesicular body. Cell. 61:623-34.

Février B, Raposo G. (2004). Exosomes: endosomal-derived vesicles shipping extracellular messages. Curr Opinion in Cell Biol. 16(4):415-21.

Friedl P, Gilmour D. (2009). Collective cell migration in morphogenesis, regeneration and cancer. Nat Rev Mol Cell Biol. 10(7):445-57.

Friess H et al. (2001). Differential expression of metastasis-associated genes in papilla of vater and pancreatic cancer correlates with disease stage. J Clin Oncol. 19(9):2422-32.

Gaggioli C et al. (2007). Fibroblast-led collective invasion of carcinoma cells with differing roles for Rho GTPases in leading and following cells. Nat Cell Biol. 9(12):1392-400.

Geradts J et al. (1999). Frequent loss of KAI1 expression in squamous and lymphoid neoplasms. An immunohistochemical study of archival tissues. Am J Path. 154(6):1665-71.

Gostner J et al. (2011). Effects of Epcam overexpression on human breast cancer cells. BMC Cancer. 11:45.

Gruenberg J. (2001). The endocytic pathway: a mosaic of domains. Nat Rev Mol Cell Biol. 2:721-30. 
Guarnieri F et al. (1993). The Tyr-X-X hydrophobic residue mediates lysosomal membrane targeting of lysosome-associated membrane protein 1. JBC. 268(3): 1941-6.

Guo X et al. (1996). KAI1 expression is up-regulated in early pancreatic cancer and decreased in the presence of metastases. Cancer Res. 56(21):4876-80.

Guo X et al. (1998). KAI-1, a new metastasis gene, is reduced in metastatic hepatocellular carcinoma. Hepat. 28(6):1481-8.

Harding C, Heuser J, Stahl P. (1983). Receptor-mediated endocytosis of transferrin and recycling of the transferrin receptor in rat reticulocytes. JCB. 97(2):329-39.

Hayes S, Seigel G. (2009). Immunoreactivity of ICAM-1 in human tumors, metastases, and normal tissues. Int J Clin Exp Pathol. 2(6):553-60.

He B et al. (2005). Tetraspanin CD82 attenuates cellular morphogenesis through down-regulating integrin alpha-6 mediated cell adhesion. JBC. 280(5):3346-54.

Hemler M. (2001). Specific tetraspanin functions. JCB. 155:1103-7.

Higashiyama M et al. (1998). KAI1/CD82 expression in nonsmall cell lung carcinoma is a novel, favorable prognostic factor: an immunohistochemical analysis. Cancer. 83(3):466-74.

Hionda Y et al. (1998). Decreased expression of the metastasis suppressor gene KAI1 in gastric cancer. Cancer Lett. 129(2):229-34.

Hislop J, von Zastrow M. (2011). Role of ubiquitination in endocytic trafficking of G-protein-coupled receptors. Traffic. 12:137-44.

Hsu C et al. (2010). Regulation of exosome secretion by Rab35 and its GTPase-activating proteins TBC1D10A-C. JCB. 189(2):223-32.

Huang C et al. (1998). Correlation of reduction in MRP-1/CD9 and KAI1/CD82 expression with recurrences in breast cancer patients. Am J Path. 153(3):973-83.

Imai Y, Sasaki T, Shinagawa Y, Akimoto K, Fujibayashi T. (2002). Expression of metastasis suppressor gene (KAI1/CD82) in oral squamous cell carcinoma and its clinico-pathological significance. Oral Oncol. 38(6):557-61.

Jackson P, Kingsley E, Russell P. (2000). Inverse correlation between KAI1 mRNA levels and invasive behavior in bladder cancer cell lines. Cancer Lett. 156(1):9-17. 
Jeanes A, Gottardi Y. (2008). Cadherins and cancer: how does cadherin dysfunction promote tumor progression? Oncogene. 27(55):6920-9.

Jee $\mathrm{B}$ et al. (2007). Effect of KAI/CD82 on the $\beta 1$ integrin maturation in highly migratory carcinoma cells. BBRC. 359:703-8.

Kang S, Creswell P. (2002), Regulation of intracellular trafficking of Human CD1d by association with MHC class II molecules. EMBO. 21(7):1650-60.

Katzmann D, Odorizzi G, Emr S. (2002). Receptor downregulation and multivesicular-body sorting. Nat Rev Mol Cell Biol. 3:893-905.

Kerkhof P et al. (2001). Proteasome inhibitors block a late step in lysosomal transport of selected membrane but not soluble proteins. MBC. 12:2556-66.

Koprowski et al. (1979). Colorectal carcinoma antigens detected by hybridoma antibodies. Somatic cell Genet. 5(6): 957-71.

Lakkaraju A, Boulan-Rodriguez E. (2008). Itinerant exosomes: emerging roles in cell and tissue polarity. Trends in Cell Bio. 18(5):199-209.

Lam-Yuk-Tseung S, Picard V, Gros P. (2006). Identification of a tyrosine-based motif (YGSI) in the amino terminus of Nramp1 (Slc11a1) that is important for lysosomal targeting. JBC. 281(42):31677-88.

Lam-Yuk-Tseung S, Touret N, Grinstein S, Gros P. (2005). Carboxyl-terminus determinants of the iron transporter DMT1/SLC11A2 isoform II (-IRE/1B) mediate internalization from the plasma membrane into recycling endosomes. Biochem. 44(36):12149-59.

Li M et al. (2011). CD82 gene expression in endometrial stromal cells leads to increase of the cell invasivenesss in the endometriotic milieu. J Mol Endocrinol. 47(2):195-208.

Lin Y, Shun C, Wu M, Chen C. A novel anticancer effect of thalidomide: inhibition of intracellular adhesion molecule-1-mediated cell invasion and metastasis thru suppression of nuclear factor-kappaB. Clin Cancer Res. 12(23): 7165-73.

Liu F et al. (2000). Frequent down-regulation and lack of mutation of the KAI1 metastasis suppressor gene in epithelial ovarian carcinoma. Gynecol Oncol. 78(1):10-15.

Liu L et al. (2007). Tetraspanin CD151 promotes cell migration by regulating integrin trafficking. JBC. 282(43):31631-42. 
Liu W, Zhang X (2006). KAI1/CD82, a tumor metastasis suppressor. Cancer Lett. 240:183-94.

Lodish H et al. (2004). Molecular Cell Biology. $5^{\text {th }}$ edition. pp 707-15. New York: W.H. Freeman and Company.

Lombardi D et al. (1999). Loss of KAI1 expression in the progression of colorectal cancer. Cancer Res. 59(22):5724-31.

Marks $M$ et al. (1997). Protein sorting by tyrosine-based signals: adapting to the Ys and wherefores. Trends in Cell Bio. 7(3):124-8.

Marsh M, van Meer G. (2008). No ESCRTs for exosomes. Science. 319(5867): 1191-2.

Mathivanan S, Simpson R. (2009). ExoCarta: a compendium of exosomal proteins and RNA. Proteomics. 9:4997-5000.

Maurer C et al. (1999). Reduced expression of the metastasis suppressor gene KAI1 in advanced colon cancer and its metastases. Surgery. 126(5):869-80.

Miranti C. (2009). Controlling cell surface dynamics and signaling: how CD82/KAI1 suppresses metastasis. Cell Sign. 21(2):196-211.

Miyazaki T et al. (2000). Mutation and expression of the metastasis suppressor gene KAI1 in esophageal squamous cell carcinoma. Cancer. 89(5):955-62.

Mohan A, Nalini V, Mallikarjuna K. (2007). Expression of motility-related protein MRP1/CD9, N-cadherin, E-cadherin, alpha catenin, and beta catenin in retinoblastoma. Exp Eye Res. 84(4): 781-9.

Mukherjee S Ghosh R, Maxfield F. (1997). Endocytosis. Physiol Rev. 77(3):759-803.

Nazarenko I et al. (2010). Cell surface tetraspanin Tspan8 contributes to molecular pathways of exosome-induced endothelial cell activation. Cancer Res. 70(4):1668-78.

Odintsova E, Sugiura T, Berditchevski F. (2000). Attenuation of EGFR receptor signaling by a metastasis suppressor, the tetraspanin CD82/KAI-1. Curr Biol. 10(16):1009-12.

Odintsova E, Voortman J, Gilbert E, Berditchevski F. (2003). Tetraspanin CD82 regulates compartmentalization and ligand-induced dimerization of EGFR. J Cell Sci. 116(Pt22):4557-66.

Ohno $\mathrm{H}$ et al. (1998). The medium subunits of adaptor complexes recognize distinct but overlapping sets of tyrosine-based sorting signals. JBC. 273(40): 2591-21. 
Okochi H et al. (1997). Expression of tetra-spans transmembrane family (CD9, CD37, CD53, CD63, CD81 and CD82) in normal and neoplastic human keratinocytes: an association of CD9 with alpha 3 beta 1 integrin. Br J Dermatol. 137(6):856-63.

Osta $\mathrm{W}$ et al. (2004). EpCAM is overexpressed in breast cancer and is a potential target for breasr cancer gene therapy. Cancer Res. 64(16): 5818-24.

Ostrowski M et al. (2010). Rab27a and Rab27b control different steps of the exosome secretion pathway. Nat Cell Bio. 12(1):19-30.

Palanisamy V et al. (2010). Nanostructural and transcriptomic analyses of human saliva derived exosomes. PLoS One. 5:5(1):e8577.

Pelchen-Matthews A, Kramer B, Marsh M. (2003). Infectious HIV-1 assembles in late endosomes in primary macrophages. JCB. 162(3):443-55.

Perrais M, Chen X, Perez-Moreno M, Gumbiner BM. (2007). E-cadherin homophilic ligation inhibits cell growth and epidermal growth factor receptor signaling independently of other cell interactions. Mol Biol Cell. 8:2013-25.

Pferrer S. (2010). Two Rabs for exosome release. Nat Cell Bio. 12:3-4.

Poczatel R et al. (1999), Ep-Cam levels in prostatic adenocarcinoma and prostatic intraepithelial neoplasia. J Urol. 162(4): 1462-6.

Pols M, Klumperman J. (2009). Trafficking and function of tetraspanin CD63. Exp Cell Res. 315(9):1584-92.

Protzel C et al. (2008). Down regulation of the metastasis suppressor protein KAI1/CD82 correlates with occurrence of metastasis, prognosis and presence of HPV DNA in human penile squamous cell carcinoma. Virchows Arch. 452:369-75.

Record M et al. (2011). Exosomes as intercellular signalosomes and pharmacological effectors. Biochem Pharm. 81(10):1171-82.

Renard H, Demaegd D, Guerriat B, Morsomme P. (2010). Efficent ER exit and vacuole targeting of yeast Sna2p require two tyrosine-based sorting motifs. Traffic. 11(7):931-46.

Richardson M, Jennings L, Zhang X. (2011). Tetraspanins and tumor progression. Clin Exp Metastasis.28(3): 261-70.

Rørth P. (2011) Whence directionality: guidance mechanisms in solitary and collective cell migration. Dev Cell. 20(1):9-18. 
Rous B et al. (2002). Role of adaptor complex AP-3 in targeting wild-type and mutated CD63 to lysosomes. Mol Biol Cell. 13(3):1071-82.

Savina A et al. (2003). Exosome release is regulated by a calcium-dependent mechanism in K562 cells. JBC. 278(22):20083-90.

Schindl M et al. (2000). The impact of expression of the metastasis suppressor protein KAI1 on prognosis in invasive squamous cell cervical cancer. Anticancer Res. 20(6B):4551-5.

Schindl M, Birner P, Breitenecker G, Oberhuber G. (2008). Downregulation of KAI1 metastasis suppressor protein is associated with a dismal prognosis in epithelial ovarian cancer. Gynecol Oncol. 83(2):244-8.

Serrels A et al. (2009). Real-time study of E-cadherin and membrane dynamics in living animals: implications for disease modeling and drug development. Cancer Res 69: 2714-9.

Shen B, Wn N, Yang J, Gould S. (2011). Protein targeting to exosomes/microvesicles by plasma membrane anchors. JBC. 286(16): 14383-95.

Shibagaki N, Hanada K, Yamashita H, Shimada S, Hamada H. (1999). Overexpression of CD82 on human T cells enhances LFA-1/ICAM-1-mediated cell-cell adhesion: functional associations between CD82 and LFA-1 in T cell activation. Eur J Immunol 29(12): 4081-91.

Shrimpton A, Borowitz D, Swendel P. (1997). Cystic fibrosis mutation frequencies in upstream New York. Hum Mutat. 10(6): 436-42.

Simpson R, Jensen S, Lim J. (2008). Proteomic profiling of exosomes. Proteomics. 8(19):4083-99.

Soldati T, Schliwa M. (2006). Powering membrane traffic in endocytosis and recycling. Nat Rev Mol Cell Biol. 7(12):897-908.

Sridhar S, Miranti C. (2006). Tetraspanin KAI1/CD82 suppresses invasion by inhibiting integrin-dependent crosstalk with c-Met receptor and Src kinases. Oncogene. 25:2367-78.

Stoorvogel W, Kleijmeer K, Geuze H, Raposo G. (2002). The biogenesis and function of exosomes. Traffic. 3:321-30.

Strumane K, Berx G, Van Roy F. Cadherins in cancer. Handb Exp Pharmacol. 165:69-103. 
$\mathrm{Su} \mathrm{J}$ et al. (2004). Decreased expression of KAI1 metastasis suppressor gene is a recurrence predictor in primary pTa and pT1 urothelial bladder carcinoma. Int $\mathrm{J}$ Urol. 11(2):74-82.

Sun H, Tang Z, Zhou G, Li X. (1998). KAI1 gene expression in hetatocellular carcinoma and its relationship with intrahepatic metastasis. J Exp Clin Cancer. 17(3):307-11.

Takahashi M, Sugivra T, Abe M, Ishii K, Shirasuna K. (2007). Regulation of c-Met signaling by the tetraspanin KAI1/CD82 affects cancer cell migration. Int J Cancer. 121(9): 1919-29.

Théry C et al. (1999). Molecular characterization of dendritic cell-derived exosomes. Selective accumulation of the heat shock protein hsc73. JCB. 147:599-610.

Tonoli H, Barrett C. (2005). CD82 metastasis suppressor gene: a potential target for new therapeutics? TMM. 11(12):563-70.

Trajkovic K et al. (2008). Ceramide triggers budding of exosome vesicles into multivesicular endosomes. Science. 319:1244-7.

Traub L. (2003). Sorting it out: AP-2 and alternate clathrin adaptors in endocytic cargo selection. JBC. 163(2):203-8.

Trzpis M, McLaughlin P, de Leij L, Harmsem M. (2007). Epithelial cell adhesion molecule: more than a carcinoma marker and adhesion marker. Am J Pathol. 171(2): 386-95.

Uchida S et al. (1999). Motility-related protein (MRP-1/CD9) and KAI1/CD82 expression inversely correlate with lymph node metastasis in oesophageal squamous cell carcinoma. Br J Cancer. 79(7-8):1168-73.

Uzawa K et al. (2002). High prevalence of decreased expression of CD82 metastasis suppressor in human oral carcinogenesis. Clin Cancer Res. 8(3):828-35.

Valadi $\mathrm{H}$ et al. (2007). Exosome-mediated transfer of mRNAs and microRNAs is a novel mechanism of genetic exchange between cells. Nat Cell Bio. 9(6):654-9.

Wang S et al. (2006). Effect of an anti-CD54 (ICAM-1) monoclonal antibody (UV3) on the growth of human uveal melanoma cells transplanted heterotopically and orthotopically in SCID mice. Int J Cancer. 118(4): 932-41.

Weinberg R. (2007). The Biology of Cancer. $1^{\text {st }}$ edition. New York; London: Garland Science.

Wessling-Resnick M, Braell W. (1990). The sorting and segregation mechanism of the endocytic pathway is functional in a cell free system. JBC. 265(2):690-709. 
White A, Lamb P, Barrett J. (1998). Frequent downregulation of the KAI1 (CD82) metastasis suppressor protein in human cancer cell lines. Oncogene. 16(24):3143-9.

Wu D, Liu L, Chen L, Ding Y. (2004). KAI1 gene expression in colonic carcinoma and its clinical significances. World J Gastro. 10(15):2245-9.

Wu Q et al. (2003). Role of tumor metastasis suppressor gene KAI1 in digestive tract carcinomas and cancer cells. Cell Tissue Res. 314(2):237-59.

Xiong Y, Liang L, Yan X, Yuan S, Wei M. (2005). Expression of metastasis suppressor gene KAI1/CD82 in cervical squamous cell carcinoma and its clinical significance. Ai Zheng. 24(1):110-5.

$\mathrm{Xu} \mathrm{C}$ et al. (2009).CD82 endocytosis and cholesterol-dependent reorganization of tetraspanin webs and lipid rafts. FASEB J. 23(10):3273-88.

Yang X et al. (2001). Overexpression of KAI1 suppresses in vitro invasiveness and in vitro metastasis in breast cancer cells. Cancer Res. 61:5284-8.

$\mathrm{Yu}$ Y et al. (1997). Loss of KAI1 messenger RNA expression in both high-grade and invasive human bladder cancers. Clin Cancer Res. 3(7):1045-59.

Yunta M, Lazo P. (2003). Tetraspanin proteins as organizers of membrane microdomains and signaling complexes. Cell Signal. 15(6):559-64.

Zhang X, Lane W, Charrin S, Rubenstein E, Liu L. (2003) EWI2/PGRL associates with the metastasis suppressor KAI1/CD82 and inhibits the migration of prostate cancer cells. Cancer Res. 63(10):2665-74.

Zhou B et al. (2004). The palmitolation of metastasis suppressor KAI1/CD82 is important for its motility and invasiveness inhibitory activity. Cancer Res. 64:7455-63. 


\section{VITA}

Mekel Marie Richardson was born in New Orleans, Louisiana in 1980. She attended Jackson State University from 1998-2002 and 2003-2005 where she earned her Bachelor of Science and Master of Science in Biology, respectively. She entered the

Integrated Programs in Biomedical Sciences at the University of Tennessee Health Science Center for PhD studies. In June of 2005, she joined Dr Xin Zhang's Laboratory in the Department of Medicine at UTHSC and investigated the functional role of the YXXФ, internalization, motif on the intracellular trafficking and function of CD82. 